TRANSACTIONS OF THE

AMERICAN MATHEMATICAL SOCIETY

Volume 350, Number 7, July 1998, Pages 2553-2628

S $0002-9947(98) 01770-X$

\title{
THE CLASSIFICATION OF THE SIMPLE MODULAR LIE ALGEBRAS: VI. SOLVING THE FINAL CASE
}

\author{
H. STRADE
}

\begin{abstract}
We investigate the structure of simple Lie algebras $L$ over an algebraically closed field of characteristic $p>7$. Let $T$ denote a torus in the $p$-envelope of $L$ in Der $L$ of maximal dimension. We classify all $L$ for which every 1-section with respect to every such torus $T$ is solvable. This settles the remaining case of the classification of these algebras.
\end{abstract}

This paper is the last of a series of notes which are concerned with the problem of classifying the simple finite dimensional Lie algebras over an algebraically closed field of characteristic $p>7$. Earlier notes have provided the preparatory material and the solution of three cases out of four. Here we will solve the remaining case. Our procedure is as follows. We define the concept of an absolute toral rank $T R(K, L)$ of a subalgebra $K$ of a Lie algebra $L$ [11] and consider tori $T$ in a $p$-envelope $L_{p}$ of a simple, not necessarily restricted Lie algebra $L$ having maximal absolute toral rank $T R\left(T, L_{p}\right)$ in $L_{p}$. These tori correspond to tori of maximal dimension in the restricted simple algebras. We then consider sections $L\left(\alpha_{1}, \ldots, \alpha_{t}\right)$ of $L$ with respect to such a torus of maximal absolute toral rank. The 1-sections have been described in [11]. In [12] the possible isomorphism classes of the 2-sections have been determined. The list occurring there is exactly that of $[3,(9.1 .1)]$ (which is no surprise, since it was our goal in [12] to make the ideas of [3] work in general). In the case of restricted algebras BLOCK and WILSON reduced this list considerably by taking into account the restrictedness of the algebras in question.

In the general situation there are four cases to be distinguished, as far as methods and results are concerned. These cases are

A) Every 1-section is solvable.

B) Every 1-section is solvable or has core $\mathfrak{s l}(2)$. There are nonsolvable 1-sections.

C) There exists a nonclassical root. No 2-section has core $H(2 ; \underline{1} ; \Phi(\tau))^{(1)}$.

D) There exist a nonclassical root and a 2 -section with core $H(2 ; \underline{1} ; \Phi(\tau))^{(1)}$.

The Lie algebras of classical type are exactly those of case B) [17], the algebras occurring in case C) are of Cartan type [18], and in case D) only algebras of Hamiltonian type occur [19]. By using these and other earlier results we show first that every simple Lie algebra over an algebraically closed field of characteristic $p>7$ is classical, or of Cartan type, or every 1-section with respect to every torus of maximal toral rank is solvable. In this note we treat the latter type algebras. For this it is crucial to determine those which have absolute toral rank 3. In a first step

Received by the editors July 2, 1995 and, in revised form, December 10, 1995.

1991 Mathematics Subject Classification. Primary 17B20.

(C)1998 American Mathematical Society 
we reduce the determination of simple algebras $L$ of this type to the determination of those in which additionally there is a bijection of Cartan subalgebras (CSA) of $L$ and maximal tori of dimension 2 in a semisimple $p$-envelope $L_{p}$. The latter algebras are classified by arguments given already in [3]. Namely, we follow the lines of that paper and indicate what kind of changes (which will be shown to be of major importance only in section 8.10) are necessary to obtain that $L$ is of Cartan type. Using this information, we then determine $L$ completely. There are only two isomorphism classes of simple algebras of toral rank 3 in which every 1 -section with respect to every such torus is solvable, namely $H(2 ;(2,1) ; \Phi(\tau))^{(1)}$ and $S(3 ; \underline{1} ; \Phi(\tau))^{(1)}$. We also normalize the tori of maximal toral rank in the $p$-envelope of these algebras $(\S 5)$. Using this information, we are able to prove that the algebras in question of arbitrary toral rank are BLOCK algebras of type $L(G, 0, f)$ or special algebras $S(m ; \underline{n} ; \Phi(\tau))^{(1)}$.

The results of this note complete the proof of the result announced in [22].

\section{ACKNOWLEDGEMENT}

Parts of this paper result from intense discussions with R.L. WILsON. In agreement with him I have chosen the present procedure (as the most promising) of following the pattern of [3]. In $\S 5$ WILSON pointed out to me that the algebras of type $S(3 ; \underline{1} ; \Phi(\tau))^{(1)}$ do occur. Also the treatment of case $(\mathbf{H} 4)$ in $\S 5$ is an outcome of these discussions. Some main ideas of $\S 7$, in particular the introduction of the algebra $V(G, T)$, Theorem 7.1, and Lemmas 7.2, 7.3 are due to him. I deeply appreciate his contributions to this note.

A.A. PRemet read the manuscript very carefully and made several helpful suggestions. In particular, he found out that there is some uncertainty in the application of the recognition theorem in [3, section 8] (which has an impact on the present manuscript since this strongly refers to methods used in [3]). Also, in the course of our joint work on the classification for the small characteristics 5 and 7 it occurred to PREMET and me that section 8 of [3] needs corrections. I am very grateful for PREMET's comments.

\section{Preliminaries}

For the sake of completeness we shall recall the main definitions and results from preceding papers. We consider in this note solely finite dimensional Lie algebras over an algebraically closed field $F$ of characteristic $p>7$. For all presupposed definitions and all facts stated without proofs and other references we refer to [21] and [3].

Let $G$ be a subalgebra of a Lie algebra $L$. Consider any $p$-envelope $\left(L_{p},[p], i\right)$ of $L$ and let $G_{p}$ denote the restricted subalgebra of $L_{p}$ generated by $i(G)$. Then

$$
T R(G, L):=\max \left\{\operatorname{dim} T \mid T \text { is a torus of }\left(G_{p}+C\left(L_{p}\right)\right) / C\left(L_{p}\right)\right\}
$$

is called the (absolute) toral rank of $G$ in $L$.

$G$ is said to act triangulably on a $G$-module $V$, if $G^{(1)}$ acts nilpotently on $V$.

We consider $L$ contained in a $p$-envelope $L_{p}$. Then we have $L_{p}^{(1)} \subset L$, whence $L$ is an ideal of $L_{p}$ and $L_{p}$ acts on $L$. In particular, if $T$ denotes a torus in $L_{p}, L$ decomposes into root spaces with respect to $T$,

$$
L=\sum_{\alpha \in \Gamma} L_{\alpha}(T), \quad \Gamma \subset T^{*} .
$$


Definition 1 ([3], [12]). Let $L$ be a Lie algebra and $T$ a torus in some $p$-envelope $L_{p}$. A root $\alpha \neq 0$ (with respect to $T$ ) is called proper, if there is $i \neq 0$ such that $\alpha\left(\left[L_{i \alpha}, L_{-i \alpha}\right]\right)=0$. A torus $T$ is called optimal, if it has maximal absolute toral rank in $L_{p}$ and if among all these tori the number of proper roots with respect to $T$ is maximal.

If $T$ is an optimal torus, then all roots are proper $([12,(5.3)])$ and $C_{L_{p}}(T)$ acts triangulably ([12, (3.1)]). Enlarging $T$ by the maximal torus of the center of $L_{p}$, we may assume that $T$ is optimal and maximal.

We shall use the notation $H:=C_{L}(T)$. In [17, p. 587] we extended the notion of a root: Let $\kappa, \mu$ be roots and $x \in L_{\mu}$. Then $x$ decomposes in $L_{p}$ into a semisimple part $x_{s}$ and a $[p]$-nilpotent part $x_{n}, x=x_{s}+x_{n}$. The semisimple part $x_{s}$ is contained in the $[p]$-subalgebra generated by $x^{[p]}$. Hence it is contained in $T$. Thus one can extend $\kappa$ to $x$ by setting $\kappa(x):=\kappa\left(x_{s}\right)$. We denote by $\Gamma(L, T)=\Gamma$ the set of all (extended) roots including 0.

For convenience we introduce the following notation: for $\alpha_{1}, \ldots, \alpha_{k} \in T^{*}$ put

$$
\left\langle\alpha_{1}, \ldots, \alpha_{k}\right\rangle:=\sum_{1 \leq i \leq k} G F(p) \alpha_{i} .
$$

A subalgebra $G$ is said to be a $k$-section with respect to $T$, if there are $G F(p)$ independent roots $\alpha_{1}, \ldots, \alpha_{k}$ such that $G=\sum_{\alpha \in\left\langle\alpha_{1}, \ldots, \alpha_{k}\right\rangle} L_{\alpha}$. For $k$-sections generated by $\alpha_{1}, \ldots, \alpha_{k}$ we use the notation

$$
L\left(\alpha_{1}, \ldots, \alpha_{k}\right):=\sum_{\alpha \in\left\langle\alpha_{1}, \ldots, \alpha_{k}\right\rangle} L_{\alpha} .
$$

Proposition $1.1([17])$. Let $T$ denote a maximal torus or a torus of maximal absolute toral rank in some $p$-envelope $L_{p}$ of a Lie algebra $L$, and $\Gamma$ the set of extended roots on $\mathcal{S}:=\bigcup_{\mu \in \Gamma} L_{\mu} \cup C_{L_{p}}(T)$.

1) For $\alpha, \beta \in \Gamma$ and $x \in L_{\alpha}$, the space $L_{\beta, \alpha}:=\sum_{i \in G F(p)} L_{\beta+i \alpha}$ is invariant under $\operatorname{ad} x$ ad $x$ has the unique eigenvalue $\beta(x)$ on this space. Thus if $\beta(x)=$ 0 then $\operatorname{ad} x$ acts nilpotently on $L_{\beta, \alpha}$. If $\beta(x) \neq 0$ then $\operatorname{ad} x$ acts invertibly on $L_{\beta, \alpha}$.

2) If $\alpha, \mu \in \Gamma$ and $x \in L_{\alpha}$ is an element satisfying $\mu(x) \neq 0$, then $\left[L_{\mu}, x\right]=L_{\mu+\alpha}$ and $\operatorname{dim} L_{\mu+\alpha}=\operatorname{dim} L_{\mu}$.

3) If $\alpha \in \Gamma$ and $x \in L_{\alpha}$ is an element satisfying $\mu(x)=0$ for all $\mu \in \Gamma$, then ad $x$ is a nilpotent endomorphism.

4) If $\alpha \in \Gamma$ is a root such that $\left[L_{\alpha}, L_{-\alpha}\right]$ acts nilpotently on $L$, then every root $\mu$ is linear on $L_{\alpha}$.

We obtain as an easy consequence

Lemma 1.2. Let $T$ denote a maximal torus or a torus of maximal absolute toral rank in some p-envelope $L_{p}$ of a Lie algebra $L$, and $\Gamma$ the set of extended roots on $\mathcal{S}:=\bigcup_{\mu \in \Gamma} L_{\mu} \cup C_{L_{p}}(T)$. Suppose that $I$ is a T-invariant ideal of $L$, and $\kappa, \mu \in \Gamma$ with $\kappa\left(I_{\mu}\right) \neq 0$. Then $L_{\kappa} \subset I$.

Proof. Proposition 1.1(2) shows that for $x \in I_{\mu}$ with $\kappa(x) \neq 0$ one has

$$
L_{\kappa}=\left[L_{\kappa-\mu}, x\right] \subset I \text {. }
$$


Denote by $A(m ; \underline{1}):=F\left[X_{1}, \ldots, X_{m}\right] /\left(X_{1}^{p}, \ldots, X_{m}^{p}\right)$ the truncated polynomial ring and by $W(m ; \underline{1}):=\operatorname{Der} A(m ; \underline{1})$ the $m$-th JACOBSON-WitT algebra. $A(m ; \underline{1})$ has nilpotent ideals

$$
A(m ; \underline{1})_{(k)}:=\sum_{a_{1}+\cdots+a_{m} \geq k}\left(\prod x_{i}^{a_{i}}\right) A(m ; \underline{1}) .
$$

Let $G$ be any Lie algebra and $\left(S_{i}^{\prime}\right)_{1 \leq i \leq t}$ the family of all minimal ideals of the semisimple quotient $G / \operatorname{rad} G$. These ideals form a direct sum $S^{\prime}=\bigoplus_{i} S_{i}^{\prime} \subset$ $G / \operatorname{rad} G$, which is called the socle of $G / \operatorname{rad} G$. According to R.E. BLocK's theorem each $S_{i}^{\prime}$ is of the form $S_{i}^{\prime}=S_{i} \otimes A\left(m_{i} ; \underline{1}\right)$, where every $S_{i}$ is a simple algebra. We call $S=\bigoplus_{i} S_{i}$ the core of $G$. [25] and [17, (1.8)] show that the only possible cores of 1 -sections $L(\alpha)$ with respect to a torus of maximal toral rank are $(0), \mathfrak{s l}(2), W(1 ; \underline{1}), H(2 ; \underline{1})^{(2)}$. We call $\alpha$ respectively solvable, classical, Witt, or Hamiltonian (cf. [3]).

Under some circumstances the socle of a semisimple Lie algebra is semisimple in its own right. As a slight generalization of $[18,(1.14)]$ we prove

Lemma 1.3. Let $G$ be a Lie algebra, $U=\bigoplus S_{i} \otimes A\left(m_{i} ; \underline{1}\right)$ the direct sum of some minimal ideals of $G$, where every $S_{i}$ is a simple algebra. Let $M$ denote a nilpotent subalgebra of $\sum\left(\operatorname{Der} S_{i}\right) \otimes A\left(m_{i} ; \underline{1}\right)$ normalizing $\operatorname{ad}_{U} G$, and $V$ the Fitting-0-space of $M$ on $\operatorname{ad}_{U} G$. If $\left[V,\left(\operatorname{ad}_{U} U\right) \cap V\right]$ acts nilpotently on $U$ but $\left(\operatorname{ad}_{U} U\right) \cap V$ does not, then $m_{i}=0$ for all $i$.

Proof. Put

$$
I_{i}:=S_{i} \otimes A\left(m_{i} ; \underline{1}\right)_{(1)}, \quad I=\sum I_{i}
$$

and assume $I \neq\{0\}$. Observe that $\operatorname{ad}_{U} G \subset \sum\left(\operatorname{Der} S_{i}\right) \otimes A\left(m_{i} ; \underline{1}\right)+V$, as $\sum\left(\operatorname{Der} S_{i}\right) \otimes A\left(m_{i} ; \underline{1}\right)$ is an ideal in Der $U$. Since $\sum\left(\operatorname{Der} S_{i}\right) \otimes A\left(m_{i} ; \underline{1}\right)$ normalizes $I$, the space $J:=\sum_{j \geq 0} V^{j}(I)$ is an ideal of $G$ which is contained in $U$. In addition, $J \cap\left(S_{i} \otimes A\left(m_{i} ; \underline{1}\right)\right)$ contains the maximal ideal $I_{i}$ of $S_{i} \otimes A\left(m_{i} ; \underline{1}\right)$. Since the latter is a minimal ideal of $G$, we obtain $S_{i} \otimes A\left(m_{i} ; \underline{1}\right) \subset J$ for all $i$, i.e., $J=U=\sum S_{i} \otimes A\left(m_{i} ; \underline{1}\right)$.

Now $M$ acts on every $I_{i}$. Decompose $U=\sum U_{\mu}$ and $I_{i}=\sum I_{i} \cap U_{\mu}$ into root spaces with respect to $M$. We obtain

$$
U_{0}=J_{0}=\sum_{i} \sum_{j \geq 0} V^{j}\left(I_{i} \cap U_{0}\right) \subset \sum_{i} I_{i} \cap U_{0}+V\left(U_{0}\right) .
$$

By assumption, $\operatorname{ad}_{U}\left(V\left(U_{0}\right)\right)=\left[V,\left(\operatorname{ad}_{U} U\right) \cap V\right]$ consists of nilpotent transformations. The same is true for every $\operatorname{ad}_{U}\left(I_{i} \cap U_{0}\right)$, whence $\left(\operatorname{ad}_{U} U\right) \cap V=\operatorname{ad}_{U} U_{0}$ acts nilpotently on $U$. This contradiction yields $I=0$ and $m_{i}=0$ for all $i$.

We need some specific information about the algebra $H(2 ; \underline{1} ; \Phi(\tau))^{(1)}$. To derive this we refer to [16] and the presentation of $H(2 ; \underline{1} ; \Phi(\tau))^{(1)}$ given there.

Let $A(2 ; \underline{1})=F[x, y], x^{p}=y^{p}=0$, denote the truncated polynomial ring in two generators, and define a distinguished element $\Lambda:=1-x^{p-1} y^{p-1}$. Then $A(2 ; \underline{1})$ carries a Lie algebra structure via

$$
\{f, g\}=\left(\left(\partial_{1} f\right)\left(\partial_{2} g\right)-\left(\partial_{1} g\right)\left(\partial_{2} f\right)\right) \Lambda \quad \text { with } \quad \partial_{1}=\partial / \partial x, \partial_{2}=\partial / \partial y
$$

The algebra $(A(2 ; \underline{1}),\{\})$ is isomorphic to $H(2 ; \underline{1} ; \Phi(\tau))$, as it is defined in [3] (cf. [14, Theorems VII.1 and VII.2] ). 
We have $(A(2 ; \underline{1}),\{\})^{(1)} \cap F 1=(0)$, and $(A(2 ; \underline{1}),\{\})^{(1)}$ is a simple Lie algebra of dimension $p^{2}-1$. For every $f \in(A(2 ; \underline{1}),\{\})$ the mapping $\{f, ?\}$ is a derivation of the truncated polynomial ring, and so there is a homomorphism $D_{\Phi(\tau)}:(A(2 ; \underline{1}),\{\}) \rightarrow W(2 ; \underline{1}), D_{\Phi(\tau)}(f)=\{f, ?\} . D_{\Phi(\tau)}(f)$ is given by

$$
D_{\Phi(\tau)}(f)=\Lambda \partial_{1}(f) \partial_{2}-\Lambda \partial_{2}(f) \partial_{1} \text {. }
$$

Obviously, ker $D_{\Phi(\tau)}=F 1$. Therefore $D_{\Phi(\tau)}$ is injective on $(A(2 ; \underline{1}),\{\})^{(1)}$. We abbreviate for the moment $\mathcal{H}:=(A(2, \underline{1}),\{\})$.

\section{Proposition 1.4.}

1) $\operatorname{Der} H(2 ; \underline{1} ; \Phi(\tau))^{(1)} \cong D_{\Phi(\tau)}(\mathcal{H}) \oplus F x^{p-1} \partial_{2} \oplus F y^{p-1} \partial_{1}$.

2) Der $H(2 ; \underline{1} ; \Phi(\tau))^{(1)}$ is a minimal p-envelope of $H(2 ; \underline{1} ; \Phi(\tau))^{(1)}$.

3) $H(2 ; \underline{1} ; \Phi(\tau))^{(1)}$ has no subalgebra of codimension 1 , and $D_{\Phi(\tau)}(\mathcal{H}) \cap$ $W(2 ; \underline{1})_{(0)}$ is the only subalgebra of codimension 2.

4) $D_{\Phi(\tau)}(\mathcal{H}) \cap W(2 ; \underline{1})_{(0)}$ is a restricted subalgebra of Der $H(2 ; \underline{1} ; \Phi(\tau))^{(1)}$.

5) $F\left(x \partial_{1}-y \partial_{2}\right)=F D_{\Phi(\tau)}(x y)$ is a 1-dimensional maximal torus of

$$
\text { Der } H(2 ; \underline{1} ; \Phi(\tau))^{(1)} \text {. }
$$

Every maximal torus of dimension 1 is conjugate under an automorphism of Der $H(2 ; \underline{1} ; \Phi(\tau))^{(1)}$ to this torus.

Proof. 1) follows from [16], Proposition 1.1(1).

2) $[16,(1.1 .(2))]$ yields that $\operatorname{Der} H(2 ; \underline{1} ; \Phi(\tau))^{(1)}$ is a $p$-envelope. Since it is centerless, it is a minimal $p$-envelope.

3) follows from $[16,(1.4 .(1))]$.

4) is straightforward.

5) We have

$$
D_{\Phi(\tau)}(x y)=\left(1-x^{p-1} y^{p-1}\right)\left(y \partial_{2}-x \partial_{1}\right)=-\left(x \partial_{1}-y \partial_{2}\right) .
$$

This is a toral element. As

$$
\begin{aligned}
\left.C_{\text {Der } H(2 ; 1 ; \Phi}(\tau)\right)^{(1)} & \left(F\left(x \partial_{1}-y \partial_{2}\right)\right) \\
=\operatorname{span} & \left(\left\{D_{\Phi(\tau)}\left(x^{i} y^{i}\right) \mid 0<i<p-1\right\} \cup\left\{D_{\Phi(\tau)}(\Lambda)\right\}\right) \\
& +F x^{p-1} \partial_{2}+F y^{p-1} \partial_{1} \\
=F D_{\Phi(\tau)}(x y) & \\
& +\left(\operatorname{span}\left(\left\{D_{\Phi(\tau)}\left(x^{i} y^{i}\right) \mid 1<i<p-1\right\} \cup\left\{D_{\Phi(\tau)}(\Lambda)\right\}\right)\right. \\
& \left.+F x^{p-1} \partial_{2}+F y^{p-1} \partial_{1}\right)
\end{aligned}
$$

acts triangulably and the latter space acts nilpotenly, $F\left(x \partial_{1}-y \partial_{2}\right)$ is a maximal torus.

We are now going to prove that every 1-dimensional torus is conjugate to this torus. Let $T=F t$ denote any maximal 1-dimensional torus and $t$ a toral element. Set $N^{i}:=\operatorname{span}\left\{x^{k} y^{l} \mid k+l \geq i\right\}, N:=N^{1}$.

We are going to construct suitable generators for $F[x, y]$.

Since ad $t$ acts semisimply, and all eigenvalues lie in $G F(p)$, there are elements $u_{1}, u_{2} \in N$ with the following properties:

(i) $t\left(u_{i}\right)=s_{i}\left(\delta_{i}+u_{i}\right), \quad s_{i} \in G F(p), \delta_{i} \in\{0,1\}$,

(ii) $u_{1}, u_{2}$ are linearly independent $\left(\bmod N^{2}\right)$. 
As a consequence, $u_{1}, u_{2}$ are generators for $F[x, y]$.

Suppose that $s_{1} \delta_{1} \neq 0$. If $\delta_{2} \neq 0$ then choose $k \in G F(p)$ with $s_{1} k=s_{2}$ and set

$$
u_{2}^{\prime}:=\left(u_{2}+1\right)-\left(u_{1}+1\right)^{k} \in N \text {. }
$$

Then

$$
\begin{aligned}
u_{2}^{\prime} & \equiv u_{2}-k u_{1} \quad\left(\bmod N^{2}\right), \\
t\left(u_{2}^{\prime}\right) & =s_{2}\left(u_{2}+1\right)-k s_{1}\left(u_{1}+1\right)^{k}=s_{2} u_{2}^{\prime} .
\end{aligned}
$$

Therefore we may assume $\delta_{2}=0$.

It is well-known that for generators $u_{1}, u_{2}$

$$
d:=\left\{u_{1}, u_{2}\right\} \not \equiv 0 \quad(\bmod N) .
$$

Thus $d$ is invertible in $F[x, y]$, and the inverse is a nonzero multiple of $d^{p-1}$. Since $t$ is a derivation with respect to $\{$,$\} , we have$

$$
\begin{aligned}
t(d) & =t\left(\left\{u_{1},, u_{2}\right\}\right)=\left\{t\left(u_{1}\right), u_{2}\right\}+\left\{u_{1}, t\left(u_{2}\right)\right\} \\
& =s_{1}\left\{1+u_{1}, u_{2}\right\}+s_{2}\left\{u_{1}, u_{2}\right\}=\left(s_{1}+s_{2}\right)\left\{u_{1}, u_{2}\right\}=\left(s_{1}+s_{2}\right) d .
\end{aligned}
$$

Put

$$
u_{2}^{\prime \prime}:=u_{2} d^{p-1} \in N
$$

Then

$$
\begin{gathered}
u_{2}^{\prime \prime} \in F u_{2}+N^{2}, \quad u_{2}^{\prime \prime} \notin N^{2}, \\
t\left(u_{2}^{\prime \prime}\right)=t\left(u_{2}\right) d^{p-1}-u_{2} d^{p-2} t(d)=\left(s_{2}-\left(s_{1}+s_{2}\right)\right) u_{2}^{\prime \prime}=-s_{1} u_{2}^{\prime \prime} .
\end{gathered}
$$

Thus we may assume $s_{2}=-s_{1}$ and $t(d)=0$.

Write $d=\sum_{i, j} \beta_{i j}\left(1+u_{1}\right)^{i} u_{2}^{j}$. Then

$$
0=t(d)=s_{1} \sum_{i, j}(i-j) \beta_{i j}\left(1+u_{1}\right)^{i} u_{2}^{j} .
$$

Consequently there are $\gamma_{i}:=\beta_{i, i}$ with

$$
d=\sum_{i=0}^{p-1} \gamma_{i}\left(1+u_{1}\right)^{i} u_{2}^{i}, \quad \gamma_{0} \neq 0 .
$$

By adjusting $u_{2}$ we may assume $\gamma_{0}=1$. Put

$$
v_{0}:=\left(1+u_{1}\right) u_{2} \equiv u_{2} \quad\left(\bmod N^{2}\right) .
$$

Then $t\left(v_{0}\right)=0$ and

$$
\left\{u_{1}, v_{0}\right\}=\left\{u_{1},\left(1+u_{1}\right) u_{2}\right\}=\left(1+u_{1}\right)\left\{u_{1}, u_{2}\right\} \equiv 1 \quad(\bmod N) .
$$

Having for $k<p-1$ inductively constructed $v_{k}$ with the properties

$$
\begin{gathered}
v_{k} \equiv u_{2} \quad\left(\bmod N^{2}\right), \quad t\left(v_{k}\right)=0, \\
\left\{u_{1}, v_{k}\right\}=\left(1+u_{1}\right)\left(1+\lambda_{k} v_{k}^{k}+\text { terms in } N^{k+1}\right)
\end{gathered}
$$

for a suitable $\lambda_{k} \in F$, we put

$$
v_{k+1}:=v_{k}-(k+1)^{-1} \lambda_{k} v_{k}^{k+1}
$$

and obtain

$$
\left\{u_{1}, v_{k+1}\right\}=\left(1+u_{1}\right)(1+f)
$$


with $f \in N^{k+1}$. Moreover, $t(f)=0$, and therefore, writing $f$ as a polynomial in $u_{1}$ and $v_{k+1}$, we see that it is a polynomial in $v_{k+1}$ only. By induction there is $v=v_{p-1}$ and $\alpha \in F$ such that

$$
\begin{gathered}
v \equiv u_{2} \quad\left(\bmod N^{2}\right), \quad t(v)=0, \\
\left\{u_{1}, v\right\}=\left(1+u_{1}\right)\left(1+\alpha v^{p-1}\right) .
\end{gathered}
$$

As $1 \notin(A(2 ; \underline{1}),\{,\})^{(1)}$ and $\left\{u_{1}, v\left(1+u_{1}\right)^{p-1}\right\}=\left(1+\alpha v^{p-1}\right)$, we obtain $\alpha \neq 0$. Put $r:=\left(\operatorname{ad} u_{1}\right)^{p}$. It is easily checked that

$$
\begin{aligned}
r\left(u_{1}\right) & =0, \\
r(v) & =-\alpha\left(1+\alpha v^{p-1}\right), \\
r^{p}(v) & =-\alpha^{p}(-\alpha)\left(1+\alpha v^{p-1}\right) .
\end{aligned}
$$

Thus $r^{p}=-\alpha^{p} r \neq 0$ is a semisimple derivation. Moreover,

$$
\begin{aligned}
(\operatorname{ad} v)^{p}\left(u_{1}\right) & =(-1)^{p}\left(1+u_{1}\right)\left(1+\alpha v^{p-1}\right)^{p}=-\left(1+u_{1}\right), \\
(\operatorname{ad} v)^{p}(v) & =0 .
\end{aligned}
$$

Thus $t=-s_{1}(\operatorname{ad} v)^{p}, r \notin F t,[r, t]=0$, whence $T$ is not a maximal torus. This contradiction shows that $s_{1} \delta_{1}=0$.

By symmetry we have $s_{1} \delta_{1}=s_{2} \delta_{2}=0$. Now if $s_{i}=0$ then we may (by definition!) assume that $\delta_{i}=0$. Thus there are generators $u_{1}, u_{2}$ such that $\delta_{1}=$ $\delta_{2}=0$. Repeating an earlier argument, we may replace $u_{2}$ by $u_{2} d^{p-1}$ to obtain $s_{2}=-s_{1} \in G F(p)$. By an adjustment of $t$ we obtain $s_{1}=1$.

Therefore there are generators $u_{1}, u_{2} \in N$ satisfying

$$
\begin{gathered}
t\left(u_{1}\right)=u_{1}, \quad t\left(u_{2}\right)=-u_{2}, \\
\left\{u_{1}, u_{2}\right\} \equiv 1 \quad(\bmod N) .
\end{gathered}
$$

Suppose for $k<p-1$ we have constructed inductively an element $u_{1, k}$ satisfying

$$
\begin{gathered}
u_{1, k} \equiv u_{1} \quad\left(\bmod N^{2}\right), \quad t\left(u_{1, k}\right)=u_{1, k}, \\
\left\{u_{1, k}, u_{2}\right\}=1+\lambda_{k} u_{1, k}{ }^{k} u_{2}{ }^{k}+\text { terms in } N^{2 k+1}
\end{gathered}
$$

for a suitable $\lambda_{k} \in F$. We put

$$
u_{1, k+1}:=u_{1, k}-(k+1)^{-1} \lambda_{k} u_{1, k}^{k+1} u_{2}^{k}
$$

and obtain

$$
\left\{u_{1, k+1}, u_{2}\right\}=(1+f)
$$

with $f \in N^{2 k+1}$. Moreover $t(f)=0$ and therefore, writing $f$ as a polynomial in $u_{1, k+1}$ and $u_{2}$, we see that it is a polynomial in $u_{1, k+1} u_{2}$ only. By induction there are $u \in N$ and $\alpha \in F \backslash\{0\}$ such that

$$
\begin{gathered}
u \equiv u_{1} \quad\left(\bmod N^{2}\right), \quad t(u)=u, \\
\left\{u, u_{2}\right\}=1+\alpha u^{p-1} u_{2}^{p-1} .
\end{gathered}
$$

Choose $\delta \in F$ with $\delta^{-p+1}=\alpha$. The mapping

$$
u^{i} u_{2}^{j} \mapsto \delta^{i+j-1} x^{i} y^{j}
$$

establishes an automorphism $\Psi$ of $(A(2 ; \underline{1}),\{\}),([10])$. This extends to an automorphism of Der $H(2 ; \underline{1} ; \Phi(\tau))^{(1)}$, which maps $t$ onto $\tilde{t}=\Psi \circ t \circ \Psi^{-1}$. Then

$$
\begin{aligned}
\tilde{t}\left(x^{i} y^{j}\right) & =\Psi\left(t\left(\delta^{1-i-j} u^{i} u_{2}^{j}\right)\right) \\
& =(i-j) \delta^{1-i-j} \Psi\left(u^{i} u_{2}^{j}\right)=(i-j) x^{i} y^{j} .
\end{aligned}
$$

Thus $t$ is conjugate to $x \partial_{x}-y \partial_{y}$. 
Definition 2. A Lie algebra $L$ is said to have property $(A ; m)$ if

a) $T R(L)=m$,

b) for every torus $T$ in some $p$-envelope $L_{p}$ of $L$ with $T R\left(T, L_{p}\right)=m$ and every root $\alpha$ with respect to $T$ the 1 -section $L_{p}(\alpha)$ is solvable.

Note that, if $L$ has property $(A ; m)$ then every torus of maximal dimension is optimal. 1-sections and 2-sections of an algebra satisfying $(A ; m)$ have been determined in $[17,(2.6)]$ :

Theorem 1.5. Let $L$ be a simple Lie algebra over an algebraically closed field of characteristic $p>7$. Assume that all 1-sections of $L$ with respect to an optimal torus $T$ are solvable. Then:

1) $C_{L}(T)$ acts nilpotently on $L$.

2) Every 1-section is nilpotent and acts triangulably on $L$.

3) Every 2-section either has core $H(2 ; \underline{1} ; \Phi(\tau))^{(1)}$ (then the absolute toral rank of this 2-section is 2) or is solvable (then the absolute toral rank is $\leq 1$ ).

4) There is a 2-section with core $H(2 ; \underline{1} ; \Phi(\tau))^{(1)}$.

5) Every 1-section which does not act nilpotently on $L$ is contained in a 2-section with core $H(2 ; \underline{1} ; \Phi(\tau))^{(1)}$.

The main mean result of [3] is a list of restricted semisimple algebras of toral rank 2 having some additional properties. We need a slight generalization of this result.

Theorem 1.6. Let $A$ be a semisimple Lie algebra over an algebraically closed field $F$ of characteristic $p>7$. Assume that $T R(A) \leq 2$. If A has a triangulable CSA $H$ then $A$ is one of the following:

1) $A=(0)$;

2) $A \in\{\mathfrak{s l}(2), W(1 ; \underline{1})\}$;

3) $S_{1} \oplus S_{2} \subset A \subset\left(\operatorname{Der} S_{1}\right)^{(1)} \oplus\left(\operatorname{Der} S_{2}\right)^{(1)}, S_{1}, S_{2} \in\left\{\mathfrak{s l}(2), W(1 ; \underline{1}), H(2 ; \underline{1})^{(2)}\right\}$;

4) $H(2 ; \underline{1})^{(2)} \subset A \subset \operatorname{Der} H(2 ; \underline{1})^{(2)}$;

5) $S \otimes A(m ; \underline{1}) \subset A \subset \operatorname{Der}(S \otimes A(m ; \underline{1})), S \in\left\{\mathfrak{s l}(2), W(1 ; \underline{1}), H(2 ; \underline{1})^{(2)}\right\}, m \neq 0$;

6) $S \subset A \subset \operatorname{Der} S$,

$S \in\left\{W(1 ; \underline{2}), H(2 ;(2,1))^{(2)}, H(2 ; \underline{1} ; \Phi(\tau))^{(1)}, H(2 ; \underline{1} ; \Delta)\right\} ;$

7) $A=S+H, \quad S \in\left\{A_{2}, C_{2}, G_{2}, W(2 ; \underline{1}), S(3 ; \underline{1})^{(1)}, H(4 ; \underline{1})^{(2)}, K(3 ; \underline{1})\right\}$.

Proof. If $T R(H, A)=0$ then $A=H$ is nilpotent, i.e., $A=(0)$. Suppose that $T R(H, A)=1$. Then $A$ has a minimal ideal $S$ such that $S \subset A \subset$ Der $S$ and $S \in\left\{\mathfrak{s l}(2), W(1 ; \underline{n}), H(2 ; \underline{n} ; \Psi)^{(2)}\right\}([25])$. Since $T R(S) \leq T R(A)=2$ we have $S \in\left\{\mathfrak{s l}(2), W(1 ; \underline{1}), W(1 ; \underline{2}), H(2 ; \underline{1})^{(2)}, H(2 ;(2,1))^{(2)}, H(2 ; \underline{1} ; \Phi(\tau))^{(1)}, H(2 ; \underline{1} ; \Delta)\right\}$. These algebras are listed in the theorem.

Suppose that $T R(H, A)=2$. Let $A_{p}$ denote a semisimple $p$-envelope of $A$, and $T$ the maximal torus contained in the $p$-envelope of $H$. Then $\operatorname{dim} T=T R(H, A)=2$, and hence $T$ is a maximal torus in $A_{p}$. Since $A$ is semisimple, it can be described as

$$
S=\bigoplus_{i=1}^{r} S_{i} \otimes A\left(m_{i} ; \underline{1}\right) \subset A \subset \sum_{i=1}^{r} \operatorname{Der}\left(S_{i} \otimes A\left(m_{i} ; \underline{1}\right)\right),
$$


where $S_{i}$ are simple algebras. Since none of these is nilpotent, we obtain

$$
r \leq \sum_{i=1}^{r} T R\left(S_{i}\right) \leq T R(A)=2 .
$$

Hence either $r=2, T R\left(S_{i}\right)=1$, or else $r=1, T R(S) \leq 2$. Let $S_{p}$ denote the $p$-envelope of $S$ in $A_{p}$. If $r=2$ or $r=1$ and $T R(S)=2$, then [17, (1.2.(5))] yields $T \subset S_{p} \subset \sum\left(\operatorname{Der} S_{i}\right) \otimes A\left(m_{i} ; \underline{1}\right)$.

In case $r=2$ we now proceed as in [3] to obtain

$$
S_{1} \oplus S_{2} \subset A \subset\left(\operatorname{Der} S_{1}\right)^{(1)} \oplus\left(\operatorname{Der} S_{2}\right)^{(1)}, S_{i} \in\left\{\mathfrak{s l}(2), W(1 ; \underline{1}), H(2 ; \underline{1})^{(2)}\right\} .
$$

In case $r=1, T R(S)=2$ we refer to the classification of these algebras ([12]) and obtain the result as well.

In case $r=1, T R(S)=1, T \subset(\operatorname{Der} S) \otimes A(m ; 1)$ we have $S \in\{\mathfrak{s l}(2), W(1 ; \underline{1})$, $\left.H(2 ; \underline{1})^{(2)}\right\}$. The kernel of the mapping

$$
\psi:(\operatorname{Der} S) \otimes A(m ; 1) \rightarrow(\operatorname{Der} S) \otimes A(m ; 1) /(\operatorname{Der} S) \otimes A(m ; 1)_{(1)} \cong \operatorname{Der} S
$$

is $p$-nilpotent. Hence $\psi$ maps $T$ injectively into Der $S$ :

$$
S+\psi(T) \subset \operatorname{Der} S .
$$

As $T R(S)=1$ we have $S \neq \operatorname{Der} S$. Thus $S=H(2 ; \underline{1})^{(2)}$, and $S \cap \psi(T) \neq(0)$. Then $C_{S \otimes A(m ; \underline{1})}(T)$ acts non-nilpotently. Lemma 1.3 applies with $M:=T$ and yields $m=0$.

In case $r=1, T R(S)=1, T \not \subset(\operatorname{Der} S) \otimes A(m ; 1)$ we have $m \neq 0$. All these algebras are listed in the theorem.

We need some detailed information about the switching of tori. Let $L$ denote a simple Lie algebra, $L_{p}$ a semisimple $p$-envelope of $L$ and $T$ a maximal torus in $L_{p}$. We may switch $T$ by use of WinTER's exponential map ([27]): Take a nonzero root $\alpha \in T^{*}$ and $x \in L_{\alpha}$. Define an abelian subalgebra of $L_{p}$ by $E^{x}(T):=\{t-\alpha(t) x \mid t \in$ $T\}$. Choose a toral element $t_{0} \in T$ with $\alpha\left(t_{0}\right)=1$. The $p$-envelope of $E^{x}(T)$ is described as

$$
E^{x}(T)_{p}=T \cap \operatorname{ker} \alpha+F\left(t_{0}-x\right)+\sum_{i \geq 1} F x^{[p]^{i}} .
$$

Note that $\alpha$ vanishes on $V:=\sum_{i \geq 1} F x^{[p]^{i}}$, since $T$ is a maximal torus and $x \in L_{\alpha}$. Thus the semisimple elements of the $[p]$-invariant space $V$ are contained in $T \cap$ ker $\alpha$. Let

$$
u:=t_{0}-x+\sum_{i \geq 1} \gamma_{i} x^{[p]^{i}}
$$

denote the semisimple part of $t_{0}-x$. Then $u^{[p]}-u \in V \cap T \subset T \cap \operatorname{ker} \alpha$. Consequently,

$$
T \cap \operatorname{ker} \alpha+F u=: e^{x}(T)
$$

is the unique maximal torus of $E^{x}(T)_{p}$. Its dimension is given by

$$
\operatorname{dim} e^{x}(T)=\operatorname{dim} T \text {. }
$$

Next let $z \in C_{L}\left(e^{x}(T)\right)$. As $T \cap \operatorname{ker} \alpha \subset e^{x}(T)$, one has $z=h+\sum_{i \neq 0} v_{i \alpha}, v_{i \alpha} \in L_{i \alpha}$, $h \in C_{L}(T)$. Then

$$
0=\left[h+\sum_{i \neq 0} v_{i \alpha}, t_{0}-x+\sum_{j>0} \gamma_{j} x^{[p]^{j}}\right] \equiv-\sum i v_{i \alpha} \quad\left(\bmod L(\alpha)^{(1)}\right),
$$


proving $v_{i \alpha} \in L(\alpha)^{(1)}$ for all $i$. Thus

$$
C_{L}\left(e^{x}(T)\right) \subset C_{L}(T)+L(\alpha)^{(1)} .
$$

If $T$ is a torus of maximal dimension then $e^{x}(T)$ has maximal dimension as well. In this case tori $T, T^{\prime}$ are called conjugate under WINTER's exponentials if $T$ is transformed into $T^{\prime}$ by a finite number of WinTER's exponentials. This definition establishes an equivalence relation on the set of tori of $L_{p}$ of maximal dimension.

Proposition 1.7. Let $L$ be a simple Lie algebra over an algebraically closed field of characteristic $p>7$. Then $L$ is classical, or of Cartan type, or satisfies $(A ; T R(L))$.

Proof. Let $L_{p}$ denote a semisimple $p$-envelope of $L$ and $T \subset L_{p}$ a torus of maximal dimension (i.e., $\operatorname{dim} T=T R(L)$ ). We refer to the proof of the fact that every optimal torus has only proper roots $([12,(5.3)])$. We have stated there that the proof of $[3,(10.4 .1)]$ applies to $L$ without any changes. The reason for this is that the proof only needs knowledge about the possible 2 -sections with respect to an optimal torus. The list used in the proof of that result in [3] is the same as we have in the general case [17]. In the proof of the theorem in [3] a little bit more has been shown than stated, namely, that every torus of maximal dimension is conjugate under WinTER's exponentials to an optimal torus. Thus there is an optimal torus $T^{\prime} \subset L_{p}$ conjugate to $T$. Let $T=T_{1}, \ldots, T_{l}=T^{\prime}$ denote a sequence of maximal tori where $T_{i-1}$ is obtained by switching $T_{i}$. If there is a nonsolvable root with respect to $T^{\prime}$ then earlier results ([17], [18], [19]) show that $L$ is classical or of Cartan type. Otherwise, due to Theorem $1.5 C_{L}\left(T^{\prime}\right)$ acts nilpotently on $L$ and every 1-section is nilpotent. Therefore $C_{L}\left(T^{\prime}\right)+L(\alpha)^{(1)}$ acts nilpotently on $L$ for every root $\alpha$ with respect to $T^{\prime}$ (by Theorem 1.5(2) and the Engel-Jacobson theorem). The reasoning preceding this proposition implies that $C_{L}\left(T_{l-1}\right)$ acts nilpotently on $L$ as well. Then every root with respect to $T_{l-1}$ is solvable, and hence $T_{l-1}$ is optimal as well. By induction, $T$ has only solvable roots. Consequently, $L$ is classical, or of Cartan type, or every torus of maximal dimension has only solvable roots.

\section{Structural features of Simple Lie algebras satisfying $(A ; 3)$}

We consider Lie algebras satisfying $(A ; 3)$, and in them maximal tori of dimension 2 .

Proposition 2.1. Let $L$ be simple satisfying $(A ; 3)$, and let $L_{p}$ be a semisimple p-envelope of $L$. Then $L_{p}$ contains maximal tori of dimension 2.

Proof. Let $T$ denote a 3 -dimensional torus in $L_{p}$. Theorem 1.5 ensures that there is a 2 -section $L(\alpha, \beta)$ with core $H(2 ; \underline{1} ; \Phi(\tau))^{(1)}$. Choose a toral element $t \in T \cap$ ker $\alpha \cap$ ker $\beta$. Next we refer to Proposition 1.4. Observe that, as Der $H(2 ; \underline{1} ; \Phi(\tau))^{(1)}$ is a $p$-envelope of $H(2 ; \underline{1} ; \Phi(\tau))^{(1)}$, there is a homomorphism from $L_{p}(\alpha, \beta)$ onto Der $H(2 ; \underline{1} ; \Phi(\tau))^{(1)}$. Choose a 1 -dimensional maximal torus $F \bar{r}$ contained in Der $H(2 ; \underline{1} ; \Phi(\tau))^{(1)}$ and lift this to a 1-dimensional torus $\operatorname{Fr}$ in $L_{p}(\alpha, \beta)$. Then $[t, r]=0$, and hence $F r \oplus F t$ is a torus.

Suppose that $F r \oplus F t$ is not a maximal torus in $L_{p}$, i.e., there is a 3 -dimensional torus $R$ which contains $F r \oplus F t$. As $L(\alpha, \beta)=C_{L}(t), L(\alpha, \beta)$ is a 2-section even with respect to $R$. Since $L(\alpha, \beta) / \operatorname{rad} L(\alpha, \beta)$ contains a subalgebra isomorphic to $H(2 ; \underline{1} ; \Phi(\tau))^{(1)}$, then $L(\alpha, \beta)$ has toral rank 2 . Hence $R$ is contained in $L(\alpha, \beta)_{p}+$ $F t$, where $L(\alpha, \beta)_{p}$ is the $p$-envelope of $L(\alpha, \beta)$ in $L_{p}([17,(1.5 .(2))])$. Consequently, 
$R$ stabilizes $\operatorname{rad} L(\alpha, \beta)$ and $R$ acts on $H(2 ; \underline{1} ; \Phi(\tau))^{(1)}$ as a 2-dimensional torus. This, however, contradicts the fact that $F \bar{r}$ is a 1-dimensional maximal torus.

Theorem 2.2. Let $L$ be simple satisfying $(A ; 3)$. Suppose $U$ is a 2-dimensional maximal torus in some semisimple p-envelope of $L$, and let $L(\alpha)$ be a 1-section with respect to $U$. Then either $L(\alpha)$ is solvable, or else

1) $\operatorname{rad} L(\alpha)$ is $U$-invariant,

2) there is an algebra $S \in\left\{\mathfrak{s t}(2), W(1 ; \underline{1}), H(2 ; \underline{1})^{(2)}, H(2 ; \underline{1} ; \Phi(\tau))^{(1)}\right\}$ such that $S \subset L(\alpha) / \operatorname{rad} L(\alpha) \subset(\operatorname{Der} S)^{(1)}$.

Moreover, the action of $U$ on $L(\alpha) / \operatorname{rad} L(\alpha)$ gives rise to a homomorphism of $U$ into Der $S$, and $\bar{U}:=\operatorname{ad}_{S} U$ is a 1-dimensional maximal torus of the p-envelope $S_{p}$ of $S$ in $\operatorname{Der} S$.

Proof. If $C_{L}(U)$ acts nilpotently on $L(\alpha)$, then (as $U$ is a maximal torus) the extended root $\alpha$ vanishes on $\bigcup_{i \in G F(p)} L_{i \alpha}$. In this case $L(\alpha)$ is nilpotent.

Thus assume that $H:=C_{L}(U)$ acts non-nilpotently on $L(\alpha)$. Then there is $h \in$ $H$ with $\alpha(h) \neq 0$. We obtain that $H$ is a CSA of $L(\alpha)$ and that for every $t \in U$ there is an element $\sum \gamma_{i} h^{[p]^{i}} \in U, \gamma_{i} \in F$, such that $t_{0}:=t-\sum \gamma_{i} h^{[p]^{i}} \in(\operatorname{ker} \alpha) \cap U$. Then $\left[t_{0}, L(\alpha)\right]=(0)$. Consequently, $\operatorname{rad} L(\alpha)$ is invariant under $U$. [11, (3.5)] yields that $H$ acts triangulably on $L$. Let $\pi: U+L(\alpha) \rightarrow(U+L(\alpha)) / \operatorname{rad} L(\alpha)$ denote the canonical homomorphism.

If $\alpha(\pi(h))=0$ then

$$
\begin{aligned}
\pi\left(\sum_{i \neq 0} L_{i \alpha}\right) & =\pi\left(\left[\sum_{i \neq 0} L_{i \alpha}, h\right]\right)=\left[\sum_{i \neq 0} \pi\left(L_{i \alpha}\right), \pi(h)\right] \\
& =\sum_{i \neq 0} i \alpha(\pi(h)) \pi\left(L_{i \alpha}\right)=(0) .
\end{aligned}
$$

In this case $\sum_{i \neq 0} L_{i \alpha} \subset$ ker $\pi$, whence $L(\alpha)$ is solvable.

Otherwise,

$$
0 \neq T R(\pi(H), \pi(L(\alpha))) \leq T R(H, L(\alpha)) \leq T R\left(U, L_{p}(\alpha)\right)=1 .
$$

In that case $\pi(L(\alpha))$ contains the triangulable CSA $\pi(H)$ of absolute toral rank 1 in $\pi(L(\alpha))$.

Let $S=\bigoplus S_{i}$ denote the socle of $\pi(L(\alpha))$, where $\bigoplus S_{i}$ is the sum of all minimal ideals. Every $S_{i}=\bigoplus_{j} S_{i} \cap \pi\left(L_{j \alpha}\right)$ is cyclicly graded. Since $S_{i}$ is not solvable, then $S_{i} \cap \pi(H)$ acts non-nilpotently [12, (1.5)]. Since $\pi(H)$ acts triangulably, Lemma 1.3 shows that every $S_{i}$ is a simple algebra. It also follows that $T R\left(S_{i} \cap \pi(H), S_{i}\right) \neq 0$. As $T R(\pi(H), \pi(L(\alpha)))=1$, this implies that $S=S_{1}$ is simple, and $S \cap \pi(H)$ is a CSA of $S$. The classification of these algebras ([25]) yields

$$
S \in\left\{\mathfrak{s l}(2), W(1 ; \underline{n}), H(2 ; \underline{n} ; \Psi)^{(2)}\right\} .
$$

Next we observe that

$$
0 \neq T R(S) \leq T R(L(\alpha)) \leq T R(L)-1=2 .
$$

If $T R(S)=1$, then $S \in\left\{\mathfrak{s l}(2), W(1 ; \underline{1}), H(2 ; \underline{1})^{(2)}\right\}$.

Suppose $T R(S)=2$. Let $t \neq 0$ denote any fixed element of $U \cap(\operatorname{ker} \alpha)$. Let $R$ denote a 2 -dimensional torus in the $p$-envelope of $S$ in $L_{p}(\alpha) / \operatorname{rad} L_{p}(\alpha)$. [12, (1.2)] gives rise to a torus $T \subset L_{p}(\alpha)$ which is mapped under the homomorphism $L_{p}(\alpha) \rightarrow L_{p}(\alpha) / \operatorname{rad} L_{p}(\alpha)$ onto $R$ and which commutes with $t$. Thus $T+F t$ is a 
torus of $L_{p}$ of dimension 3. Now $L$ satisfies $(A ; 3)$. Then $C_{L}(T+F t)=C_{L(\alpha)}(T)$ acts nilpotently on $L$. Therefore $C_{S}(R)$ acts nilpotenly on $S$, which shows that every root on $\mathrm{S}$ with respect to $R$ is solvable. Theorem 1.5 yields $S \cong H(2 ; \underline{1} ; \Phi(\tau))^{(1)}$.

In the cases $S \cong \mathfrak{s l}(2)$ or $\cong W(1 ; \underline{1})$ we have Der $S \cong S \cong(\operatorname{Der} S)^{(1)}$. To deal with the case $S \cong H(2 ; \underline{1})^{(2)}$ we recall, that $\alpha(S \cap \pi(H)) \neq 0$. Thus $\pi(L(\alpha))=$ $S+\pi(H) \cap(\operatorname{ker} \alpha) \subset \operatorname{Der} S$. This is only true if $\pi(L(\alpha)) \subset H(2 ; \underline{1})=(\operatorname{Der} S)^{(1)}$. In case $S=H(2 ; \underline{1} ; \Phi(\tau))^{(1)}$ the derivation algebra Der $H(2 ; \underline{1} ; \Phi(\tau))^{(1)}$ is linearly spanned by $H(2 ; \underline{1} ; \Phi(\tau))^{(1)}$ and a 2 -dimensional torus $R$. If $\pi(L(\alpha)) \neq S$ then $R \cap \pi(L(\alpha)) \neq(0)$. But then there is a 3 -dimensional torus $T$ in $L_{p}$ such that $C_{L}(T)$ acts nonnilpotently on $L$. This contradicts Theorem 1.5. Therefore we have $\pi(L(\alpha))=S=(\operatorname{Der} S)^{(1)}$.

Finally, we observe that $\pi(U)$ acts on $\pi(L(\alpha))^{(2)}=S$. Thus the restriction $\bar{U}=\operatorname{ad}_{S} \pi(U)$ is a torus in Der $S$. Choose a toral element $r$ in the $p$-envelope of $\pi(H) \cap S$ in $S_{p}$ with $\alpha(r)=1$. Since $\pi(U) \cap(\operatorname{ker} \alpha)$ centralizes $S$, we have $\bar{U}=\operatorname{ad}_{S} F r$. Suppose that $F r$ is not a maximal torus in $S_{p}$. As above, $U$ then would be contained in a 3 -dimensional torus of $L_{p}$, contradicting the maximality of $U$.

With respect to a 2-dimensional maximal torus we now have an additional type of 1-section: there are solvable, classical, Hamiltonian 1-sections $\left(S \cong H(2 ; \underline{1})^{(2)}\right)$, and those for which $S$ is a BLOCK algebra

$$
S \cong H(2 ; \underline{1} ; \Phi(\tau))^{(1)} \cong L(G, 0, f)^{(1)} \text { with }|G|=p^{2} .
$$

We will in the following refer to the latter as BLOCK 1-sections and call the corresponding roots BLOCK roots.

Corollary 2.3. Let $L$ be simple satisfying $(A ; 3)$. Let $U$ be a 2-dimensional maximal torus in $L_{p}, \alpha$ an improper root and $x \in L_{\alpha}$. Then $e^{x}(U)$ is a 2-dimensional maximal torus.

Proof. Suppose, first, that $\alpha$ is a Block root. Then the core of $L(\alpha)$ is isomorphic to $H(2 ; \underline{1} ; \Phi(\tau))^{(1)}$, and $U$ acts on $H(2 ; \underline{1} ; \Phi(\tau))^{(1)}$ as a 1 -dimensional maximal torus of Der $H(2 ; \underline{1} ; \Phi(\tau))^{(1)}$ (cf. Theorem 2.2). Proposition 1.4 shows that it acts as $D_{\Phi(\tau)}(x y)$. Therefore $\alpha$ is a proper root, showing that this case is impossible.

Suppose now that $e^{x}(U)$ is contained in a 3-dimensional torus $R$. Let $t \in U$ be a toral element which spans $U \cap(\operatorname{ker} \alpha)$. Then $F t \subset e^{x}(U) \subset R$, and $L(\alpha)=C_{L}(t)$ is a 2 -section with respect to $R$. Theorem 1.5 yields, as $\alpha$ is not a BLOCK root, that $L(\alpha)$ is solvable. Then $L(\alpha)$ is a proper root, a contradiction.

Theorem 2.4. Let $L$ be simple satisfying $(A ; 3)$, and let $L_{p}$ be a semisimple $p$ envelope of $L$. Then either $L$ is of Cartan type $H(2 ; \underline{n} ; \Psi)^{(2)}$, or every maximal 2-dimensional torus $U \subset L_{p}$ has the property that $C_{L}(U)$ is a CSA of $L$ and $U$ is contained in the p-envelope of $C_{L}(U)$ in $L_{p}$.

Proof. Let $U$ be a maximal 2-dimensional torus in $L_{p}$. The maximality of $U$ ensures that $C_{L}(U)$ is nilpotent.

Suppose that there is a root $\alpha$ vanishing on $C_{L}(U)$. Then the 1 -section $L(\alpha)$ is nilpotent as well. Let $\beta$ denote any root independent of $\alpha$ and give $L$ the grading $L_{i}=\sum_{j \in G F(p)} L_{j \alpha+i \beta}$. Then $L_{0}=L(\alpha)$ does not act nilpotently on $L$, since otherwise $L$ would be solvable. Consequently, $\beta\left(\bigcup_{i} L_{i \alpha}\right) \neq 0$, and $L(\alpha)$ is a CSA of 
absolute toral rank 1 in $L$. The classification of these algebras shows (as $L \not \mathfrak{s l}(2)$ ) that $L$ is of Cartan type $W(1 ; \underline{n})$ or $H(2 ; \underline{n} ; \Psi)^{(2)}$.

Next we assume that no root vanishes on $C_{L}(U)$. Then $C_{L}(U)$ is a CSA of $L$. If the $p$-envelope $M$ of $C_{L}(U)$ in $L_{p}$ does not contain $U$, then the unique maximal torus of $M$ is properly contained in $U$, and hence has dimension 1 . In that case, $C_{L}(U)$ has absolute toral rank 1 in $L$ and, as above, $L$ is of Cartan type $W(1 ; \underline{n})$ or $H(2 ; \underline{n} ; \Psi)^{(2)}$.

It has been proved in $[14,(\mathrm{~V} .2),(\mathrm{V} .3),(\mathrm{V} .4)]$ that every maximal torus $R$ in a semisimple $p$-envelope $W(1 ; \underline{n})_{p}$ of $W(1 ; \underline{n})$ has the property that $C_{W(1 ; \underline{n})}(R)$ acts non-nilpotently. Thus Theorem 1.5(1) shows that no algebra of type $W(1 ; \underline{n})$ satisfies $(\mathrm{A} ; 3)$.

Next we are going to derive a lower bound for $\operatorname{dim} L$.

Lemma 2.5. Suppose $B$ is a restricted solvable Lie algebra and $R$ is a maximal torus of $B$ of toral rank 2. Let $M$ denote a $B$-module with representation $\rho$. Assume that there are roots $\beta, \gamma$ with respect to $R$, such that $\gamma \notin G F(p) \beta$ and $\rho\left(\left[B_{\gamma}, B_{\beta-\gamma}\right]\right)$ contains non-nilpotent transformations. Then there is $\lambda \notin G F(p) \beta$ and $x_{\lambda} \in B_{\lambda}$ with $\rho\left(x_{\lambda}\right)^{p-1} \neq 0$.

Proof. (1) Suppose in a first step that $B$ contains a $R$-invariant nilpotent subalgebra $K$ such that

$$
\begin{aligned}
& K=\sum_{\mu \notin G F(p) \beta} K_{\mu}+\sum_{\lambda, \mu \notin G F(p) \beta}\left[K_{\lambda}, K_{\mu}\right], \\
& \rho\left(K^{(1)}\right) \text { contains a non-nilpotent transformation. }
\end{aligned}
$$

As $K$ is nilpotent, there is a member $N:=K^{n}$ of the descending central series such that

$$
\begin{aligned}
& \rho([[N, K], K]) \text { consists of nilpotent transformations, } \\
& \rho([N, K]) \text { contains a non-nilpotent transformation. }
\end{aligned}
$$

Decompose $N=\bigoplus N_{\mu}$ with respect to $R$. We may assume that $\rho\left(x_{\mu}\right)^{p-1}=0$ for all $x_{\mu} \in N_{\mu}, \mu \notin G F(p) \beta$, because otherwise the lemma is true.

For $y_{\lambda} \in K_{\lambda}, x_{\mu} \in N_{\mu}, \mu \notin G F(p) \beta$ one has (cf. [21, (5.7.1)])

$$
\begin{aligned}
0 & \left.=[\underbrace{\rho\left(y_{\lambda}\right), \ldots,\left[\rho\left(y_{\lambda}\right)\right.}_{(p-1) \text { times }}, \rho\left(x_{\mu}\right)^{p-1}] \ldots\right] \\
& \equiv(p-1) ! \rho\left(\left[y_{\lambda}, x_{\mu}\right]\right)^{p-1} \quad(\bmod [[N, K], K] U(K)) .
\end{aligned}
$$

Note that $[[N, K], K] U(K)$ is an ideal of $U(K)$. The choice of $N$ ensures that $\rho([[N, K], K] U(K))$ consists of nilpotent transformations only. Thus $\rho\left(\left[y_{\lambda}, x_{\mu}\right]\right)$ is nilpotent for all roots $\lambda$, and all $\mu \notin G F(p) \beta$.

Next suppose $\lambda, \mu \in G F(p) \beta$. The assumption on $K$ yields

$$
y_{\lambda} \in \sum_{\kappa \notin G F(p) \beta}\left[K_{\kappa}, K_{\lambda-\kappa}\right] .
$$

Hence

$$
\left[y_{\lambda}, x_{\mu}\right] \in \sum_{\kappa \notin G F(p) \beta}\left[\left[K_{\kappa}, x_{\mu}\right], K_{\lambda-\kappa}\right] \subset[[N, K] K] .
$$

Thus $\rho\left(\left[y_{\lambda}, x_{\mu}\right]\right)$ is nilpotent.

If $\lambda \notin G F(p) \beta, \mu \in G F(p) \beta$, then $\left[y_{\lambda}, x_{\mu}\right] \in N_{\lambda+\mu}$ with $\lambda+\mu \notin G F(p) \beta$. The above assumption yields that $\rho\left(\left[y_{\lambda}, x_{\mu}\right]\right)$ is a nilpotent transformation. 
These results show, as $\rho([[N, K], K])$ consists of nilpotent transformations, that $\rho\left(\left[y_{\lambda}, x_{\mu}\right]\right)$ is nilpotent in any case. From this one concludes, by Jacobson's theorem on nil Lie sets, that $[N, K]$ acts nilpotently, a contradiction.

(2) We are now going to construct a subalgebra $K$ satisfying the assumptions of (1). Since $R$ is maximal, we have the notion of extended roots available. Then $\beta\left(\left[B_{\mu}, B_{i \beta-\mu}\right]\right)=0$ for all $i \neq 0$. If there were $i \neq 0$ and $\mu \notin G F(p) \beta$ with $\mu\left(\left[B_{\mu}, B_{i \beta-\mu}\right]\right) \neq 0$, then, as

$$
(i \beta-\mu)\left(\left[B_{\mu}, B_{i \beta-\mu}\right]\right)=-\mu\left(\left[B_{\mu}, B_{i \beta-\mu}\right]\right) \neq 0,
$$

an application of Proposition 1.1 and induction would yield that $B_{\mu}, B_{i \beta-\mu} \subset B^{(n)}$ for all $n$. This would contradict the solvability of $B$. As $T R(R, B)=2$ and hence the root lattice is spanned by $\beta$ and $\mu$, this implies

$$
\kappa\left(\left[B_{\mu}, B_{i \beta-\mu}\right]\right)=0 \quad \forall \mu \notin G F(p) \beta, \forall i \in G F(p) \backslash(0), \forall \kappa .
$$

If there is $\mu \notin G F(p) \beta$ such that $\rho\left(\left[B_{\mu}, B_{-\mu}\right]\right)$ contains a non-nilpotent transformation, then we set

$$
K:=\sum_{i \neq 0} B_{i \mu}+\sum_{i \neq 0}\left[B_{i \mu}, B_{-i \mu}\right] .
$$

As $B$ is solvable, then $K$ is nilpotent. This is the result.

Thus we may assume that $\rho\left(\left[B_{\mu}, B_{-\mu}\right]\right)$ consists of nilpotent transformations only. Set

$$
K:=\sum_{\mu \notin G F(p) \beta} B_{\mu}+\sum_{\lambda, \mu \notin G F(p) \beta}\left[B_{\lambda}, B_{\mu}\right] .
$$

We observe that $K_{i \beta}=\sum_{\mu \notin G F(p) \beta}\left[B_{i \beta-\mu}, B_{\mu}\right]$. According to the above we may assume that $\bigcup_{\mu \notin G F(p) \beta}\left[B_{i \beta-\mu}, B_{\mu}\right]$ consists of ad-nilpotent elements. We clearly also may assume that $\rho$ is faithful, and $\rho\left(x_{\mu}\right)^{p-1}=0$ for all $\mu \notin G F(p) \beta, x_{\mu} \in$ $K_{\mu}$. Then $\rho\left(\left(\operatorname{ad} x_{\mu}\right)^{p}(B)\right)=\left[\rho\left(x_{\mu}\right)^{p}, \rho(B)\right]=(0)$, whence $\left(\operatorname{ad} x_{\mu}\right)^{p}(B)=(0)$ and $\left(\operatorname{ad} x_{\mu}\right)^{p}=0$. Similarly we obtain $(\operatorname{ad} h)^{p^{r}}=0$ for all $h \in \bigcup_{\mu \notin G F(p) \beta}\left[K_{\mu}, K_{-\mu}\right]$ (for $r$ sufficently big). Thus we have proved that

$$
\bigcup_{i \neq 0 ; \mu \notin G F(p) \beta}\left[K_{i \beta-\mu}, K_{-\mu}\right] \cup \bigcup_{\mu \notin G F(p) \beta} K_{\mu} \cup \bigcup_{\mu \notin G F(p) \beta}\left[K_{\mu}, K_{-\mu}\right]
$$

consists of ad-nilpotent elements. Then $K$ is an $R$-invariant nilpotent subalgebra of $K$ (by the Engel-Jacobson theorem). Since by our assumption $\rho\left(\left[K_{\gamma}, K_{\beta-\gamma}\right]\right) \subset$ $\rho\left(K^{(1)}\right)$ does not consist of nilpotent transformations only, this final case is impossible.

Lemma 2.6 ([3]). Let $L$ be a restricted Lie algebra, $T$ a maximal torus with $T R(T, L)=2$, and $L=\sum_{\mu \in \Gamma} L_{\mu}$ the root space decomposition with respect to $T$. Suppose that there are $\alpha, \beta \in \Gamma \backslash(0), x \in L_{\alpha}, y \in L_{\beta}$ with $\alpha(y) \neq 0, \beta(x) \neq 0$. Then every faithful $L$-module $V$ has at least $p^{2}-1$ weights. In particular, if $L$ has a subalgebra isomorphic to $H(2 ; \underline{1} ; \Phi(\tau))^{(1)}$, then $\operatorname{dim} V \geq p^{2}-1$.

Proof. Decompose $V=\bigoplus_{\mu \in \Delta} V_{\mu}$ into weight spaces with respect to the abelian algebra $T$. Write $x=x_{s}+x_{n}, y=y_{s}+y_{n}$ with $x_{s}, y_{s} \in T$ the semisimple parts and $x_{n}, y_{n}$ the $[p]$-nilpotent parts of $x$ and $y$. Then $\alpha\left(y_{s}\right) \neq 0, \beta\left(y_{s}\right)=0, \alpha\left(x_{s}\right)=0$, $\beta\left(x_{s}\right) \neq 0$. Thus $T=F x_{s} \oplus F y_{s}$. 
The faithfulness of $V$ implies $x . V \neq(0), y \cdot V \neq(0)$. Thus there are weights $\gamma, \delta \in \Delta$ such that $\gamma+\alpha, \delta+\beta \in \Delta$. Since not both $\gamma, \gamma+\alpha$ vanish on $y_{s}$, there is $\mu \in \Delta$ with $\mu(y) \neq 0$. Then $V_{\mu+j \beta} \neq(0)$ for all $j \in G F(p)$. Next, there is at most one index $j_{0}$ such that $\left(\mu+j_{0} \beta\right)(x)=0$. Therefore $\mu+j \beta+i \alpha \in \Delta$ for all $j \neq j_{0}$ and all $i \in G F(p)$. Again there is at most one index $i_{0}$ such that $\left(\mu+i_{0} \alpha\right)(y)=0$. Then $V_{\mu+i \alpha+j_{0} \beta}=\left[y, V_{\mu+i \alpha+\left(j_{0}-1\right) \beta}\right] \neq(0)$ for $i \neq i_{0}$. Thus $\left\{\mu+i \alpha+j \beta \mid(i, j) \neq\left(i_{0}, j_{0}\right)\right\} \subset \Delta$.

Suppose $L$ contains a subalgebra $G$ isomorphic to $H(2 ; \underline{1} ; \Phi(\tau))^{(1)}$. Since $G$ is simple and $V$ is faithful, there is a factor $W$ of a composition series of $V$ as a $G$-module which is not annihilated by $G$. Then $W$ is a faithful $G$-module. The lemma applies to the $p$-envelope of $G$ and $W$.

We are going to apply this lemma in the following way. Suppose $L$ is simple and satisfies $(A ; 3)$. Let $T \subset L_{p}$ be a maximal torus with $T R\left(T, L_{p}\right)=3$. Assume that $L(\alpha, \beta)$ is a 2 -section with respect to $T$ with core $H(2 ; \underline{1} ; \Phi(\tau))^{(1)}$. Let $\pi$ : $L(\alpha, \beta) \rightarrow L(\alpha, \beta) / \operatorname{rad} L(\alpha, \beta)$ be the canonical homomorphism. Then

$$
\pi(L(\alpha, \beta))^{(1)} \cong H(2 ; \underline{1} ; \Phi(\tau))^{(1)},
$$

and $T$ acts on $H(2 ; \underline{1} ; \Phi(\tau))^{(1)}$ as a 2 -dimensional torus. The 2-dimensional tori in Der $H(2 ; \underline{1} ; \Phi(\tau))^{(1)}$ are well-known ([14, Theorem VII.3]). They are conjugate to $F(1+x) \partial_{1} \oplus F(1+y) \partial_{2}$. Therefore all root spaces are 1-dimensional and all root vectors act non-nilpotently. Thus, lifting this information to $L(\alpha, \beta)$, we find $x \in L_{\alpha}, y \in L_{\beta}$ with $\alpha(y) \neq 0, \beta(x) \neq 0$. Now one can apply the lemma to the restricted algebra $L_{p}(\alpha, \beta)$ and the torus $T$.

Theorem 2.7. Suppose that $L$ is simple and satisfies $(A ; 3)$. Let $V$ be a nontrivial L-module. Then

$$
\operatorname{dim} V \geq p^{3}-p
$$

Proof. Consider $L$ as a subalgebra of $\mathfrak{g l}(V)$, and let $L_{p}$ denote the $p$-envelope of $L$ in $\mathfrak{g l}(V)$. Let $T \subset L_{p}$ be a maximal torus with $T R\left(T, L_{p}\right)=3$, and $L=\bigoplus_{\mu \in \Gamma} L_{\mu}$ the root space decomposition with respect to $T$. Let $\Delta$ denote the set of weights on $V$ with respect to $T$. We may assume that $V$ is irreducible. Then for any weight $\kappa \in \Delta$ we have

$$
\Delta \subset \kappa+\langle\Gamma\rangle
$$

Due to Theorem 1.5 there is a 2 -section $L(\alpha, \beta)$ with core $H(2 ; \underline{1} ; \Phi(\tau))^{(1)}$.

Suppose that there are $\kappa \in \Delta$ and $\gamma \in \Gamma \backslash\langle\alpha, \beta\rangle$ such that, for all $i \in G F(p)$, there exist $\lambda \in \Delta \cap(\kappa+i \gamma+\langle\alpha, \beta\rangle)$ and $\mu \in\langle\alpha, \beta\rangle \backslash(0)$ satisfying $\lambda\left(L_{\mu}\right) \neq 0$.

Then we consider the $L(\alpha, \beta)$-modules $V_{i}:=\sum_{j, k \in G F(p)} V_{\kappa+i \gamma+j \alpha+k \beta}$. Since there is a weight $\lambda$ on $V_{i}$ satisfying $\lambda\left(L_{\mu}\right) \neq 0$, this is a nontrivial module. Due to Lemma 2.6 we have $\operatorname{dim} V_{i} \geq\left(p^{2}-1\right)$, which gives the assertion. Thus we have to construct a weight $\kappa$ and a root $\gamma \notin\langle\alpha, \beta\rangle$ with these properties. Set

$$
\Omega:=\left\{\mu \in T^{*} \mid \mu\left(L_{\beta}\right) \neq 0\right\} .
$$

As $L(\alpha, \beta)$ has core $H(2 ; \underline{1} ; \Phi(\tau))^{(1)}$, one concludes that $\alpha\left(L_{\beta}\right) \neq 0$, whence $\alpha \in \Omega$.

(a) Suppose that there is a root $\gamma \in \Omega \backslash\langle\alpha, \beta\rangle$ such that $L_{\gamma}$ acts non-nilpotently on $L$. Theorem 1.5(5) shows that there is a 2 -section $L(\gamma, \lambda)$ with core $H(2 ; \underline{1} ; \Phi(\tau))^{(1)}$. Then $\{i \gamma+j \lambda \mid(i, j) \neq(0,0)\} \subset \Gamma$, and since $\Gamma \subset\langle\alpha, \beta, \gamma\rangle$ we may, replacing $\lambda$ by $\lambda+i \gamma$ if necessary, adjust $\lambda$ so that $\lambda \in\langle\alpha, \beta\rangle \backslash(0)$. For any $\delta \in \Delta$ consider the $L(\gamma, \lambda)$-module $V_{(\delta)}:=\sum_{i, j \in G F(p)} V_{\delta+i \gamma+j \lambda}$ with corresponding representation $\rho_{\delta}$. 
If $\rho_{\delta}(L(\gamma, \lambda))$ were solvable for all $\delta \in \Delta$, then for some $k \in \mathbb{N} L(\gamma, \lambda)^{(k)}$ would act trivially on $V$. As $L(\gamma, \lambda)^{(k)} \neq(0)$ for all $k$, and $L$ is simple, this would imply that $V$ is a trivial $L$-module, a contradiction. Thus there is $\kappa \in \Delta$ such that $\rho_{\kappa}(L(\gamma, \lambda))$ has core $H(2 ; \underline{1} ; \Phi(\tau))^{(1)}$. Now Lemma 2.6 applies to the $p$-envelope of $\rho_{\kappa}(L(\gamma, \lambda))$ and $V_{(\kappa)}$. It proves that

$$
|(\kappa+G F(p) \gamma+G F(p) \lambda) \cap \Delta| \geq p^{2}-1
$$

In particular,

$$
|(\kappa+i \gamma+G F(p) \lambda) \cap \Delta| \geq\left(p^{2}-1\right)-(p-1) p=p-1>6
$$

for every $i \in G F(p)$. If any of these sets (for any fixed $i$ ) were to vanish on $\bigcup_{\mu \in\langle\alpha, \beta\rangle \backslash(0)} L_{\mu}$, then $\lambda$ would vanish on this set as well, contradicting the fact that $\lambda \in\langle\alpha, \beta\rangle$ and $L(\alpha, \beta)$ has core $H(2 ; \underline{1} ; \Phi(\tau))^{(1)}$. In this case $\gamma$ has the required properties. We therefore may assume that $L_{\gamma}$ acts nilpotently on $L$ for all $\gamma \in$ $\Omega \backslash\langle\alpha, \beta\rangle$.

(b) Since $L$ is simple, we have by Schue's Lemma

$$
\Gamma \subset \Omega \cup(\Omega+\Omega)
$$

proving that $\Omega \not \subset\langle\alpha, \beta\rangle$. Fix $\gamma \in \Omega \backslash\langle\alpha, \beta\rangle$. Then $\Gamma \subset\langle\alpha, \beta, \gamma\rangle$. Let $\lambda=i \alpha+j \beta+k \gamma$, $k \neq 0$, be any root with $\lambda \notin\langle\alpha, \beta\rangle$, and assume that $L_{\lambda}$ acts non-nilpotently. Then there is $\mu \in \Gamma$ such that $L(\lambda, \mu)$ has core $H(2 ; \underline{1} ; \Phi(\tau))^{(1)}$ (by Theorem 1.5.(5)). In particular, all $i \lambda+j \mu,(i, j) \neq(0,0)$, are roots, and no root space corresponding to these roots acts nilpotently on $L$. As $k \neq 0$ we may, replacing $\mu$ by $\mu-l \lambda$ if necessary, adjust $\mu$ so that $\mu \in\langle\alpha, \beta\rangle$. Now $(\lambda+G F(p) \mu) \cap\langle\alpha, \beta\rangle=\emptyset$. As all $L_{\lambda+i \mu}$ act non-nilpotently, the present assumption yields $(\lambda+G F(p) \mu) \cap(\Omega \backslash\langle\alpha, \beta\rangle)=\emptyset$. Thus

$$
(\lambda+G F(p) \mu) \cap \Omega=\emptyset .
$$

Therefore we have by definition $\lambda\left(L_{\beta}\right)=\mu\left(L_{\beta}\right)=0$. As $\mu \in\langle\alpha, \beta\rangle \backslash(0)$ and $L(\alpha, \beta)$ has core $H(2 ; \underline{1} ; \Phi(\tau))^{(1)}$, this implies $\beta=r \mu$ for some $r \neq 0$. However, since $L(\lambda, \mu)$ has core $H(2 ; \underline{1} ; \Phi(\tau))^{(1)}$ we have $\lambda\left(L_{r \mu}\right) \neq(0)$, a contradiction. As a consequence we may assume that for every root $\lambda \in \Gamma \backslash\langle\alpha, \beta\rangle$ the root space $L_{\lambda}$ acts nilpotently on $L$.

(c) As in (a), there is a weight $\kappa \in \Delta$ such that $|(\kappa+G F(p) \alpha+G F(p) \beta) \cap \Delta| \geq$ $p^{2}-1$. Replacing $\kappa$ by $\kappa+\alpha$ or $\kappa+2 \alpha$ if necessary, we may assume

$$
\kappa \in \Delta \cap \Omega \text {. }
$$

As $\kappa\left(L_{\beta}\right) \neq 0$, the simplicity of $L$ ensures the existence of a root $\mu \in \Gamma \backslash\langle\alpha, \beta\rangle$ such that

$$
\kappa\left(\left[L_{\mu}, L_{\beta-\mu}\right]\right) \neq 0
$$

Set $B$ equal to the $p$-envelope of $L(\beta, \mu)+T$ in $L_{p}$. Since by assumption of this case (c) $L_{\mu}$ acts nilpotently on $L, L(\beta, \mu)$ cannot have core $H(2 ; \underline{1} ; \Phi(\tau))^{(1)}$. So Theorem 1.5.(3) yields that $L(\beta, \mu)$ is solvable. Then $B$ is solvable as well. Now $B$ acts on the space $V_{(\kappa)}:=\sum_{i, j \in G F(p)} V_{\kappa+i \beta+j \mu}$. Denote the representation by $\rho_{\kappa}$. By choice of $\kappa$, clearly $\left[L_{\mu}, L_{\beta-\mu}\right]=\left[B_{\mu}, B_{\beta-\mu}\right]$ acts non-nilpotently on $V_{(\kappa)}$. Now Lemma 2.5 yields the existence of $\gamma \in\langle\beta, \mu\rangle, \gamma \notin G F(p) \beta$, and $x_{\gamma} \in L_{\gamma}$ such that $\rho_{\kappa}\left(x_{\gamma}\right)^{p-1} \neq 0$. 
Note that $\gamma \notin\langle\alpha, \beta\rangle$, since otherwise $L(\alpha, \beta)=L(\gamma, \beta)=L(\mu, \beta)$ would be solvable, a contradiction. Also, $\mu=r \beta+s \gamma$ with $s \neq 0$. If $\gamma\left(\left[L_{\mu}, L_{\beta-\mu}\right]\right) \neq 0$, then $\mu\left(\left[L_{\mu}, L_{\beta-\mu}\right]\right) \neq 0$ and $(\beta-\mu)\left(\left[L_{\mu}, L_{\beta-\mu}\right]\right) \neq 0$. Proposition 1.1 would imply that $L_{\mu}, L_{\beta-\mu} \subset \bigcap_{n} L(\beta, \mu)^{(n)}=(0)$, a contradiction.

Now find $\delta \in \kappa+G F(p) \beta+G F(p) \mu$ and a weight vector $v_{\delta} \in V_{\delta}$ such that $\rho_{\kappa}\left(x_{\gamma}\right)^{p-1}\left(v_{\delta}\right) \neq 0$. Then $\delta+G F(p) \gamma \subset \Delta$.

Note that $\delta$ is of the form $\delta=\kappa+i \beta+j \gamma$, and so $\delta\left(\left[L_{\mu}, L_{\beta-\mu}\right]\right)=\kappa\left(\left[L_{\mu}, L_{\beta-\mu}\right]\right) \neq$ 0. Consequently,

$$
(\delta+i \gamma)\left(L_{\beta}\right) \neq 0 \quad \forall i \in G F(p) .
$$

This proves the theorem.

We will now work step by step through $\S 5$ and $\S 6$ of [3]. We adopt the numbering of that article and refer to it by boldface numbers.

Let $S$ be a Lie algebra, $T$ a maximal torus in some $p$-envelope $S_{p}$ of $S$ such that $H:=C_{S}(T)$ acts triangulably on $S$. Let $\tilde{H}$ denote the $p$-envelope of $H$ in $S_{p}$. Observe that under these hypotheses $T+\tilde{H}$ decomposes as

$$
T+\tilde{H}=T+I
$$

where $I$ is an ideal of $T+\tilde{H}$ (containing $\tilde{H}^{(1)} \subset H^{(1)}$ ), which acts nilpotently on S. Put

$$
A:=S+\tilde{H}+T .
$$

5.1 remains valid if the word "restricted" is deleted everywhere.

5.2. We are interested in the algebras $S$ which occur as semisimple quotients of 1-sections in Theorem 2.2: In the situation of that theorem let $T$ denote the image of $U \subset L_{p}(\alpha)$ in Der $S$. Observe that, with the above definitions

$$
S \subset L(\alpha) / \operatorname{rad} L(\alpha) \subset A \subset \operatorname{Der} S .
$$

For $S \in\left\{\mathfrak{s l}(2), W(1 ; \underline{1}), H(2 ; \underline{1})^{(2)}\right\}$ we have the result of Lemma 5.2.1. A similar statement is needed for the remaining case of Theorem 2.2 , namely that $S=$ $H(2 ; \underline{1} ; \Phi(\tau))^{(1)}$.

Lemma 2.8. Suppose that

$$
H(2 ; \underline{1} ; \Phi(\tau))^{(1)} \subset A \subset \operatorname{Der} H(2 ; \underline{1} ; \Phi(\tau))^{(1)} .
$$

Let $T \subset A$ denote a maximal 1-dimensional torus of $\operatorname{Der} H(2 ; \underline{1} ; \Phi(\tau))^{(1)}$. Then $T$ is conjugate under an automorphism of $\operatorname{Der} H(2 ; \underline{1} ; \Phi(\tau))^{(1)}$ to $F\left(x \partial_{1}-y \partial_{2}\right)$. Define $\alpha \in T^{*}$ by $\alpha\left(x \partial_{1}-y \partial_{2}\right)=1$. Then $K_{\alpha}(A)$ has basis

$$
\left\{D_{\Phi(\tau)}\left(x^{i+1} y^{i}\right) \mid 2 \leq i \leq p-2\right\} \cup\left\{D_{\Phi(\tau)}\left(y^{p-1}\right)\right\},
$$

so $\operatorname{dim} A_{\alpha} / K_{\alpha}(A)=2, K_{-\alpha}(A)$ has basis

$$
\left\{D_{\Phi(\tau)}\left(x^{i} y^{i+1}\right) \mid 2 \leq i \leq p-2\right\} \cup\left\{D_{\Phi(\tau)}\left(x^{p-1}\right)\right\},
$$

so $\operatorname{dim} A_{-\alpha} / K_{-\alpha}(A)=2, K_{2 \alpha}(A)$ has basis

$$
\left\{D_{\Phi(\tau)}\left(x^{i+2} y^{i}\right) \mid 1 \leq i \leq p-3\right\} \cup\left\{D_{\Phi(\tau)}\left(x y^{p-1}\right), D_{\Phi(\tau)}\left(y^{p-2}\right)\right\},
$$

so $\operatorname{dim} A_{2 \alpha} / K_{2 \alpha}(A)=1, K_{-2 \alpha}(A)$ has basis

$$
\left\{D_{\Phi(\tau)}\left(x^{i} y^{i+2}\right) \mid 1 \leq i \leq p-3\right\} \cup\left\{D_{\Phi(\tau)}\left(x^{p-1} y\right), D_{\Phi(\tau)}\left(x^{p-2}\right)\right\},
$$

so $\operatorname{dim} A_{-2 \alpha} / K_{-2 \alpha}(A)=1$, and $K_{i \alpha}(A)=A_{i \alpha}$ if $i \neq 0, \pm 1, \pm 2$. Finally, $T \subset$ $[A,[A, I]]$. 
Proof. Proposition 1.4 shows that $T$ has the asserted property. The remaining statements are proved as in Lemma $\mathbf{5 . 2 . 1}$ (d).

5.3. We now consider the following

Setting 1:

(a) L denotes a simple Lie algebra, $L_{p}$ is a semisimple $p$-envelope of $L, T$ denotes a fixed maximal torus of dimension $2, H:=C_{L}(T)$ and $\tilde{H}$ is the $p$-envelope of $H$ in $L_{p}$. Set $A:=L+\tilde{H}$.

(b) Assume that $T \subset \tilde{H}$.

(c) Assume that every 1-section $A(\alpha)$ with respect to $T$ fulfils the assertions of Theorem 2.2.

It seems that the hypothesis (5.7.1) in [3] might not be quite weak enough to yield step (12) of Lemma 7.6.1, since in that step it seems unclear whether (in the notation of that lemma) the torus $T$ of $\bar{Q}$ has maximal dimension in $\bar{Q}$. However, appearently it is sufficient to know that the 1-sections are classical, Witt, Hamiltonian, or BLOCK to obtain all the results of section 5. This condition is now granted by assumption (c) of "Setting 1".

Lemma 2.9. Under the assumption of "Setting 1" the following are true.

1) $\tilde{H}$ acts triangulably on $L$.

2) $\tilde{H}=T \oplus I$, where $I$ is an ideal of $\tilde{H}$ which contains $\tilde{H}^{(1)}$ and acts nilpotently on $L$.

3) If $\alpha, \beta \in T^{*}$ are $G F(p)$-independent roots, then the extended roots are $F$ independent on $H$.

Proof. The CSA $H$ of $L$ acts triangulably on $L$. Then $\tilde{H}^{(1)}=H^{(1)}$ acts nilpotently as well. Since $L_{p}$ is semisimple, every element of $\tilde{H}^{(1)}$ is $[p]$-nilpotent. Let $I$ be the $p$-radical of $\tilde{H}$. Due to "Setting $\mathbf{1}$ " $T$ is a maximal torus of $\tilde{H}$. Thus $\tilde{H}=T \oplus I$. This proves 1), 2).

Suppose $\alpha, \beta$ are $F$-linearly dependent on $H$. By definition of an extended root this means that there are $t_{1}, t_{2} \in F$ (not both 0 ), such that for all $h=h_{s}+h_{n} \in H$, $h_{s} \in T, h_{n} \in I$

$$
t_{1} \alpha\left(h_{s}\right)=t_{2} \beta\left(h_{s}\right)
$$

Due to (b) of "Setting $\mathbf{1}$ " $\alpha, \beta$ are $F$-dependent on $T$. Now let $\left(u_{1}, u_{2}\right)$ be a basis of $T$ consisting of toral elements. As $\alpha\left(u_{i}\right)=\alpha\left(u_{i}^{[p]}\right)=\alpha\left(u_{i}\right)^{p}, \beta\left(u_{i}\right)=\beta\left(u_{i}\right)^{p}$, we have $\alpha\left(u_{i}\right), \beta\left(u_{i}\right) \in G F(p)$ for $i=1,2$. Then the above equation has a nontrivial solution with $t_{1}, t_{2} \in G F(p)$. Thus $\alpha, \beta$ are $G F(p)$-dependent on $T$.

Lemma 5.3.1 remains valid under the assumption of "Setting 1". In fact no changes in the proof are necessary.

Definition 5.3.2 has to become extended by a further case. As before we say that $\gamma$ is a BLOCK root if $H(2 ; \underline{1} ; \Phi(\tau))^{(1)} \subset L[\gamma] \subset \operatorname{Der} H(2 ; \underline{1} ; \Phi(\tau))^{(1)}$.

Lemma 5.3.3 is substituted by Theorem 2.2. In the following we only have to take care of the additional possibility of a root being of BLOCK type.

Lemma 5.3.4 (completed): Under the assumption of "Setting 1" the cases (a) - (f) of $[3,(5.3 .4)]$ occur and the respective statements are true, or $(g) \gamma$ is a BLOCK root, there is a surjective homomorphism $\Psi_{\gamma}$ of $A(\gamma)$ to a subalgebra of 
Der $H(2 ; \underline{1} ; \Phi(\tau))^{(1)}$ containing $H(2 ; \underline{1} ; \Phi(\tau))^{(1)}$ such that $\Psi_{\gamma}(T)=F\left(x \partial_{1}-y \partial_{2}\right)$, and there is some $j \neq 0$ such that for $i \neq 0$

$$
\operatorname{dim} A_{i \gamma} / K_{i \gamma}(A)=\left\{\begin{array}{lll}
2 & \text { if } \quad i= \pm j, \\
1 & \text { if } i= \pm 2 j, \\
0 & \text { if } \quad i \neq \pm j, \pm 2 j .
\end{array}\right.
$$

In particular, every BLOCK root is proper.

Proof. The statements are proved by the respective arguments of $[3,(5.3 .4)]$ and Lemma 2.8 .

Lemma 5.3.6 (completed). Under the assumption of "Setting 1" the cases (a), (b) of $[3,(5.3 .6)]$ occur and the respective statements are true, or (c) if $\alpha$ is BLOCK, $A(\alpha)$ contains a unique compositionally classical subalgebra of codimension 2 and no such subalgebra of codimension 1.

Proof. The proof of [3, (5.3.6)] for cases (a) and (b) remains unchanged if $A$ and $T$ are taken as above. Suppose $\alpha$ is of BLOCK type, and let $M$ denote a compositionally classical subalgebra of codimension $\leq 2$ in $A(\alpha)$. Then $\Psi_{\alpha}(M)$ is a compositionally classical subalgebra of $A[\alpha]$. Now [16, (1.4.(1))] shows that

$$
\Psi_{\alpha}(M) \cap H(2 ; \underline{1} ; \Phi(\tau))^{(1)}=H(2 ; \underline{1} ; \Phi(\tau))^{(1)} \cap W(2 ; \underline{1})_{(0)} .
$$

Thus $D_{\Phi(\tau)}(x), D_{\Phi(\tau)}(y)$ are linearly independent modulo $\Psi_{\alpha}(M)$. Then $\Psi_{\alpha}(M)=$ $A[\alpha] \cap W(2 ; \underline{1})_{(0)}$, and $M=\Psi_{\alpha}^{-1}\left(A[\alpha] \cap W(2 ; \underline{1})_{(0)}\right)$.

5.4. Under the assumption of "Setting 1 " no changes are needed in Lemma 5.4.1, while the next lemma only needs the obvious changes:

Lemma 5.4.2 (completed). Let $\gamma \neq 0$. Then

$$
\operatorname{dim} R K_{\gamma} / R_{\gamma} \leq \operatorname{dim} A_{\gamma} / K_{\gamma} .
$$

In particular, if $A_{\gamma}=K_{\gamma}$ then $R K_{\gamma}=R_{\gamma}$ and if $\gamma$ is non-Hamiltonian and nonBlock then $\operatorname{dim} R K_{\gamma} / R_{\gamma} \leq 1$.

5.5. Let $B$ be a restricted Lie algebra and let $e$ be a derivation of $B$ satisfying $e^{p}=e$. Define $\alpha \in(F e)^{*}$ by $\alpha(e)=1$. Let $B=\sum_{i \in G F(p)} B_{i \alpha}$ be the decomposition of $B$ with respect to the torus $F e$. Assume

$$
B^{(1)} \cap B_{0}=F z+J,
$$

where $z$ is central in $B$ and not $[p]$-nilpotent, and $J$ is an ideal of $B^{(1)} \cap B_{0}$ in which every element is $[p]$-nilpotent. Let $R_{i \alpha}(F e+B):=\left\{x \in B_{i \alpha} \mid\left[x, B_{-i \alpha}\right] \subset J\right\}$ and $n_{i}=\operatorname{dim} B_{i \alpha} / R_{i \alpha}(F e+B)$.

Proposition 5.5.1. Let $B$ and e as above. Let $W \neq(0)$ be a restricted $B$-module with $z W=W$. Then

(a) $\operatorname{dim} W \geq p^{m}$, where $m=\max \left\{\left[\left(n_{i}+1\right) / 2\right] \mid i \neq 0\right\}$,

(b) if $W$ is an $(F e+B)$-module and $\sum_{i \neq 0} n_{i}>2$, then $\operatorname{dim} W \geq p^{2}$.

Proof. The proof for (a) in [3, (5.5.1)] works without changes in our case, since not the decomposition of $B_{0}$ but only that of $B^{(1)} \cap B_{0}$ is needed.

In order to prove (b) we may as in the course of [3, (5.5.1)] assume that

- $W$ is an irreducible $(F e+B)$-module of dimension $p$,

- there are $i \neq 0$ and $x \in B_{i \alpha}$ such that $x^{p-1} W \neq(0)$. 
Then $W$ has $p$ weights, and each weight space is 1-dimensional. But then $B_{0}^{(1)}$ acts trivially on $W$, which means that we have a decomposition $B_{0}=F z+J^{\prime}$, where $J^{\prime} W=(0)$. Now the arguments of $[3,(5.5 .1)(\mathrm{b})]$ yield the result.

Proposition 5.5.2. Under the assumption of "Setting $\mathbf{1}$ ", for any root $\gamma \neq 0$

$$
\sum_{i \neq 0} \operatorname{dim} K_{i \gamma} / R K_{i \gamma} \leq 2, \quad \operatorname{dim} K_{\gamma} / R K_{\gamma} \leq 1
$$

Also, $\operatorname{dim} K_{\gamma} / R K_{\gamma}=1$ if and only if $\operatorname{dim} K_{-\gamma} / R K_{-\gamma}=1$.

Proof. For $0 \neq \gamma \in \Gamma$ set $n_{\gamma}:=\operatorname{dim} K_{\gamma} / R K_{\gamma}$. Choose $\alpha \in \Gamma$ so that $n_{\alpha}$ is maximal. Since $L_{p}$ is semisimple, there is some $\gamma \in \Gamma$ such that $\gamma \notin G F(p) \alpha$. Then by Schue's Lemma we have

$$
H \subset \sum_{\gamma \in \Gamma, \gamma \notin G F(p) \alpha}\left[A_{\gamma}, A_{-\gamma}\right] \subset L_{p}^{(1)} \cap C_{A}(T) \subset C_{L}(T)=H .
$$

Since $H$ acts triangulably on $L, \alpha$ is linear on $H$ (and $\alpha(H) \neq 0$ due to Lemma 2.9), and hence there exists $\beta \in \Gamma, \beta \notin G F(p) \alpha$, such that $\alpha\left(\left[A_{\beta}, A_{-\beta}\right]\right) \neq 0$. Set $W:=\left(\sum_{i \in G F(p)} A_{\beta+i \alpha}\right) /\left(\sum_{i \in G F(p)} M_{\beta+i \alpha}^{\alpha}\right)$. As in [3], we conclude that $\operatorname{dim} W \leq$ $p\left(6+n_{\alpha}\right)$.

There exists $t \in T$ such that $\alpha(t)=1, \beta(t)=0$. Set $B^{\prime}=\operatorname{ker} \alpha+I+\sum_{i \neq 0} K_{i \alpha}$, and $B$ the $p$-envelope of $B^{\prime}$ in $L_{p}$. Set $e=\operatorname{ad}_{B} t$. Proposition 5.5.1 applies and yields in combination with the arguments given in [3] that $\sum_{i \neq 0} n_{i \alpha} \leq 2$. The rest of the proof in $[3,(5.5 .2)]$ remains unchanged.

Corollary 5.5.3. Under the assumption of "Setting 1 " the following is true:

$$
\operatorname{dim} A_{\gamma} / R_{\gamma} \leq\left\{\begin{array}{llll}
1 & \text { if } & \gamma & \text { is solvable, } \\
2 & \text { if } & \gamma & \text { is classical, } \\
3 & \text { if } & \gamma & \text { is Witt, } \\
5 & \text { if } & \gamma & \text { is Hamiltonian proper } \\
7 & \text { if } & \gamma & \text { is Hamiltonian improper } \\
5 & \text { if } & \gamma & \text { is Block. }
\end{array}\right.
$$

Proof. The assertion follows from the completed versions of Lemma 5.3.4, Lemma 5.4.2 and Proposition 5.5.2.

5.6. Under the assumption of "Setting 1" there is only one additional remark to make, namely that every BLOCK root $\alpha$ is proper (cf. Lemma 5.3.4), and that $\Psi_{\alpha}(T)=F\left(x \partial_{1}-y \partial_{2}\right)$ is contained in the compositionally classical subalgebra of codimension 2 in $A[\alpha]$.

5.7. Under the assumption of "Setting 1" one has to make the obvious change in Lemma 5.7.1.(a), namely

(a) ... Furthermore, if $\left|\Gamma_{K} \cap \mathbb{Z} \gamma\right|=4$ then $\gamma$ is Hamiltonian or BLOCK, ...

The proofs of Lemmas 5.7.2 - 5.7.6 only refer to Lemma 5.7.1 and hence remain valid.

5.8. Only some particular Cartan type Lie algebras are investigated in this section.

Section 6 is the first section where we switch tori. This is now the place where we have to specify the algebras even more. It is our goal to prove that the Lie 
algebras $L$ under consideration are of Cartan type. According to Theorem 2.4 we therefore may impose an additional assumption.

\section{Setting 2:}

(a) $L$ denotes a simple Lie algebra satisfying $(A ; 3)$, and $L_{p}$ is a semisimple $p$ envelope of $L$.

(b) Every maximal 2-dimensional torus $U \subset L_{p}$ has the property that $C_{L}(U)$ is a $C S A$ of $L$ and $U$ is contained in the p-envelope of $C_{L}(U)$ in $L_{p}$.

(c) $T$ denotes a fixed maximal torus of dimension $2, H:=C_{L}(T)$, and $\tilde{H}$ is the p-envelope of $H$ in $L_{p}$.

(d) $A:=L+\tilde{H}$.

Note that this "Setting 2" covers all previous assumptions. Therefore the results of section $\mathbf{5}$ apply here. When going through section $\mathbf{6}$ of [3] we assume that $A$ satisfies the "Setting 2".

Let $A_{0}$ be a maximal subalgebra of $A$ containing $\tilde{H}$. Choose a subspace $A_{-1} \supset$ $A_{0}$ which is stable under ad $A_{0}$, and define inductively

$$
\begin{aligned}
A_{i+1} & :=\left\{x \in A_{i} \mid\left[A_{-1}, x\right] \subset A_{i}\right\} \quad \text { if } i \geq 0, \\
A_{-i-1} & :=A_{-i}+\left[A_{-1}, A_{-i}\right] \quad \text { if } i<0 .
\end{aligned}
$$

We call this the standard filtration of $A$ with respect to $A_{0}, A_{-1}$. Let $G$ := $\bigoplus A_{i} / A_{i+1}$ denote the associated graded Lie algebra.

Lemma 6.1.1 remains valid, if in (c) we replace the word "non-Hamiltonian" by "non-Hamiltonian and non-BLOCK".

Unlike in [3], we say in the case under consideration that a 2-dimensional maximal torus $T^{\prime}$ is an optimal torus, if for every 2-dimensional maximal torus $U$ we have

$$
n\left(A, T^{\prime}\right) \geq n(A, U) .
$$

Lemma 6.2.2, Corollary 6.2.3, Lemma 6.3 .2 (and their proofs) remain completely unchanged, while in Lemma 6.3.3 one has to replace the word "non-Hamiltonian" by "non-Hamiltonian and non-BLOCK". In Lemma 6.4.1 and Corollary 6.4.2 the root $\alpha$ is assumed to be nonproper, and hence is non-BLOCK. Everything (including the proofs) remains unchanged. Again, in the proof of Lemma 6.4.3 one just has to replace "Hamiltonian" by "Hamiltonian or BLOCK", while in Lemma 6.4.4 the root $\alpha$ is assumed to be nonproper. These two lemmas then remain true without changes.

As a result, section 6 of [3] remains valid if one replaces the assumptions on $A$ and $T$ there by "Setting $\mathbf{2}$ " and if one makes the obvious changes in the assertions. One should keep in mind that we are mainly interested in maximal tori of dimension 2 . This leads to a change in the notion of a proper root, namely, all solvable, classical, BLOCK roots are proper, and a Witt or Hamiltonian root $\gamma$ is proper if and only if $A_{i \gamma}=R_{i \gamma}$ for some $i \neq 0$. Let $\Gamma_{P}$ be the set of proper roots. Correspondingly, the definition of an optimal torus is slightly changed.

\section{The CASE $\operatorname{rad} G_{0} \neq(0)$}

We continue to assume that $A$ fulfils "Setting 2". We also assume that $T$ is an optimal torus. Let $A_{0}$ be a maximal subalgebra of $A$ containing $R(A)$ (i.e., a "distinguished maximal subalgebra" in the sense of [3]) and

$$
A=A_{-k} \supset \cdots \supset A_{-1} \supset A_{0} \supset A_{1} \supset \ldots
$$


the standard filtration constructed by means of $A_{0}$ and an irreducible $A_{0}$-module $A_{-1} / A_{0}$. Let $G=\sum_{i \geq-k} G_{i}$ denote the associated graded algebra. Then $T$ can be identified with its image in $G_{0}$.

Throughout this section we assume

$$
\operatorname{rad} G_{0} \neq(0)
$$

and work through section $\mathbf{7}$ of [3]. Lemmas 7.2.7, 7.3 .1 and 7.3.3 and their proofs remain valid in our case, Lemma 7.3.2 and its proof remains valid, if one replaces the word "non-Hamiltonian" by "non-Hamiltonian and non-BLOCK". Recalling that every BLOCK root is proper, we observe that Lemma 7.5.2 and its proof need no changes.

Proposition 3.1. $G_{0}$ is one of the following:

1) $\mathfrak{s l}(2) \oplus C\left(G_{0}\right)$;

2) $W(1 ; \underline{1}) \oplus C\left(G_{0}\right)$;

3) $\mathfrak{s l}(2) \oplus \mathcal{H}$, the 6-dimensional split extension of $\mathfrak{s t}(2)$ by a 3-dimensional Heisenberg algebra $\mathcal{H}$;

4) $W(1 ; \underline{1}) \oplus A(1 ; \underline{1})$, the split extension of $W(1 ; \underline{1})$ by the restricted $W(1 ; \underline{1})$ module $A(1 ; \underline{1})$ with zero multiplication;

5) $\mathfrak{s l}(2) \oplus A(1 ; \underline{1})$, the split extension of $\mathfrak{s l}(2)$ by the restricted $\mathfrak{s l}(2)$-module $A(1 ; \underline{1})$ with zero multiplication, where $\mathfrak{s l}(2)$ is considered as a subalgebra of $W(1 ; \underline{1})$.

In any case, $(0) \neq C\left(G_{0}\right) \subset T$.

Proof. As $\tilde{H}=C_{A}(T)$ is a triangulable CSA which is contained in $R(A) \subset A_{0}$, it follows that $\bar{H}:=C_{G_{0}}(T)$ is a triangulable CSA of $G_{0}$. Since $G_{0}=A_{0} / A_{1}$, we have $T R\left(\bar{H}, G_{0}\right) \leq T R(\tilde{H}, A)=2$.

Suppose that $T R\left(\bar{H}, G_{0}\right)=2$. Then there are 2 independent roots $\alpha, \beta$ with respect to $T$ on $G_{0}$. Since $\operatorname{rad} G_{0} \neq(0)$, there is a nonzero abelian ideal $J$ of $G_{0}$. Consider any root vector $x \in J_{\mu}$. Then $(\operatorname{ad} x)^{p}\left(G_{0}\right) \subset J^{(1)}=(0)$, and hence $\alpha(x)=\beta(x)=0$. Since $\alpha, \beta$ span the root lattice on $G$, this implies that $J$ acts nilpotently not only on $G_{0}$ but on $G$ and hence annihilates $G_{-1}$. By construction of the graded algebra $G$, however, $G_{0}$ contains no nonzero ideal with this property. Hence $T R\left(\bar{H}, G_{0}\right)=1$, and consequently $T \cap \operatorname{rad} G_{0}=F z \neq(0)$ is contained in the center of $G_{0}$.

As $G_{-1}$ is an irreducible $G_{0}$-module, $z$ acts on $G_{-1}$ as a multiple of the identity. Therefore $G_{-1}$ decomposes into root spaces $G_{-1}=\bigoplus_{i \in G F(p)} G_{-1, \delta+i \alpha}$ for suitable roots $\alpha, \delta$, and hence there are at most $p$ roots on $G_{-1}$. We obtain by Lemma $\mathbf{6 . 3 . 3}$

$$
\operatorname{dim} G_{-1} \leq 7 p<p^{2}-3 p \text {. }
$$

Thus "Assumption $B$ " of [13, p. 704] is fulfilled for $G_{0}$ and $\rho: G_{0} \rightarrow \mathfrak{g l}\left(G_{-1}\right)$. According to the Corollary of [13, (III.1)] one of the following is true:

a) $G_{0} / F z$ has a unique minimal ideal $S\left(G_{0}\right) / F z$, which is a simple algebra,

b) $G_{0}$ is the 6 -dimensional split extension of $\mathfrak{s l}(2)$ by a 3 -dimensional Heisenberg algebra,

c) $G_{0}$ has an abelian ideal $I \not \subset C\left(G_{0}\right)$.

To investigate case a) we observe that, as $T \cap C\left(G_{0}\right) \neq(0), G_{0}=G_{0}(\alpha)$ is a 1-section. Then it is the quotient of $A_{0} \cap A(\alpha)$ by a solvable ideal. Thus $G_{0}$ is the homomorphic image of a subalgebra of a 1-section. Theorem 2.2 implies that $S\left(G_{0}\right) / F z$ has to be a quotient of a subalgebra of $\mathfrak{s l}(2), W(1 ; \underline{1}), H(2 ; \underline{1})^{(2)}$ or 
$H(2 ; \underline{1} ; \Phi(\tau))^{(1)}$. [13, (III.4)] then shows that $G_{0}$ is of type (1) or (2) of this proposition or that $G_{0}$ contains an ideal $S$ such that $S / F z \in\left\{H(2 ; \underline{1})^{(2)}, H(2 ; \underline{1} ; \Phi(\tau))^{(1)}\right\}$. Lemma 7.2.7 of [3] in combination with Lemma 6.3.3 excludes the case $S / F z \cong$ $H(2 ; \underline{1})^{(2)}$, while in the case $S / F z \cong H(2 ; \underline{1} ; \Phi(\tau))^{(1)}$ Lemma 2.6 yields the contradiction $\operatorname{dim} G_{-1} \geq p^{2}-1$.

We now consider case c). Suppose $G_{0}$ is solvable. Then $A_{0}$ is solvable as well. Let $Q$ be a maximal subalgebra of $L$ containing $A_{0} \cap L$. Since $H \subset A_{0} \cap L \subset Q$, we have that $Q$ is invariant under $\tilde{H}$ (which is the $p$-envelope of $H$ in $L_{p}$ ). Hence $Q+\tilde{H}$ is a subalgebra of $A$, and as $A=L+\tilde{H}$, one has $A_{0} \subset Q+\tilde{H}$. The assumption $Q+\tilde{H}=A$ leads to the contradiction $L=Q+\tilde{H} \cap L=Q+H=Q$. Then the maximality of $A_{0}$ proves that $A_{0}=Q+\tilde{H}$. Consequently $Q$ would be solvable. Thus $[9,24]$ show that $L \in\{\mathfrak{s l}(2), W(1 ; \underline{n})\}$. None of these algebras satisfies $(A ; 3)$ ([14]). Thus $G_{0}$ cannot be solvable. Theorem III.3 of [13] now shows that $G_{0}$ is as in 4 ) or 5$)$.

In all cases the center of $G_{0}$ is at most 1-dimensional. We have already mentioned above that $\operatorname{dim} T \cap C\left(G_{0}\right)=1$. Then $(0) \neq C\left(G_{0}\right) \subset T$.

Lemma 3.2. 1) There is a subalgebra $K \in\{\mathfrak{s l}(2), W(1 ; \underline{1})\}$ of $G_{0}$ such that $G_{0}$ is the semidirect sum

$$
G_{0}=K \oplus \operatorname{rad} G_{0}
$$

and

$$
T=(T \cap K) \oplus C\left(G_{0}\right)
$$

2) $C_{G_{0}}(T)=T$.

Proof. In cases 1) and 2) of Proposition 3.1 we have $G_{0}=G_{0}^{(1)} \oplus C\left(G_{0}\right), C\left(G_{0}\right) \subset T$, $G_{0}^{(1)} \cong \mathfrak{s l}(2), W(1 ; \underline{1})$. The assertions follow immediately from this.

Case 3): Choose a canonical basis $(e, f, h)$ for the subalgebra isomorphic to $\mathfrak{s l}(2)$. Let $\pi: G_{0} \rightarrow G_{0} / \mathcal{H} \cong \mathfrak{s l}(2)$ denote the canonical homomorphism. If $[T, \mathcal{H}]=(0)$ then $\tilde{H}$ would contain as a subalgebra the inverse image of $\mathcal{H}$, and this subalgebra acts nontriangulably on $L$, a contradiction. Therefore $\pi(T)$ acts nontrivially on $\mathcal{H} / C\left(G_{0}\right)$. Consequently, $\mathcal{H} / C\left(G_{0}\right)$ is a 2-dimensional nontrivial $\mathfrak{s l}(2)$-module,

$$
\mathcal{H}=G_{0,1} \oplus G_{0,-1} \oplus C\left(G_{0}\right)
$$

and therefore $\operatorname{ad}_{G_{0}} h$ has eigenvalues $\pm 2, \pm 1,0$. Since $p>7$ and the extension $G_{0}=\mathfrak{s l}(2) \oplus \mathcal{H}$ splits, every $x \in \bigcup_{i \neq 0} G_{0, i}$ satisfies $\left(\operatorname{ad}_{G_{0}} x\right)^{3}=0$. Therefore $\exp (\operatorname{ad} x)$ is an automorphism of $G_{0}$. Recall that $\pi(T) \neq(0)$. It is now easy to see that there are $x_{j} \in \bigcup_{i \neq 0} G_{0, i}$ such that $\prod_{j} \exp \left(\operatorname{ad} x_{j}\right)(T)=F h \oplus C\left(G_{0}\right)$. This proves the first claim. The second claim is an immediate consequence.

Cases 4) and 5): Note that in these cases $G_{0}$ is $W(1 ; \underline{1}) \oplus A(1 ; \underline{1})$ or a homogeneous subalgebra of this. Let $t \in T \backslash C\left(G_{0}\right)$ have the property that $\left(\operatorname{ad}_{G} t\right)^{p}=\operatorname{ad}_{G} t$. Write

$$
t=t^{\prime}+f, \quad t^{\prime} \in G_{0} \cap W(1 ; \underline{1}), \quad f \in A(1 ; \underline{1}) .
$$

Then by Jacobson's identity

$$
\operatorname{ad}_{G} t=\left(\operatorname{ad}_{G} t\right)^{p}=\left(\operatorname{ad}_{G} t^{\prime}\right)^{p}+\operatorname{ad}_{G}\left(\left(\operatorname{ad} t^{\prime}\right)^{p-1}(f)\right)+\left(\operatorname{ad}_{G} f\right)^{p} .
$$


As a consequence,

$$
\begin{aligned}
\left(\operatorname{ad} t^{\prime}-\left(\operatorname{ad} t^{\prime}\right)^{p}\right)\left(G_{0} \cap W(1 ; \underline{1})\right) \subset G_{0} \cap W(1 ; \underline{1}) \cap A(1 ; \underline{1}) & =(0), \\
\left(\operatorname{ad} t^{\prime}-\left(\operatorname{ad} t^{\prime}\right)^{p}\right)(A(1 ; \underline{1})) \subset[A(1 ; \underline{1}), A(1 ; \underline{1})] & =(0), \\
(\operatorname{ad} f)^{p}\left(G_{0}\right) \subset[A(1 ; \underline{1}), A(1 ; \underline{1})] & =(0) .
\end{aligned}
$$

Since $G_{-1}$ is an irreducible $G_{0}$-module, we conclude that

$$
\operatorname{ad}_{G_{-1}} t^{\prime}-\left(\operatorname{ad}_{G_{-1}} t^{\prime}\right)^{p}, \quad\left(\operatorname{ad}_{G_{-1}} f\right)^{p} \in F \operatorname{Id}_{G_{-1}} .
$$

Also $F 1 \subset A(1 ; \underline{1})$ acts nontrivially on $G_{-1}$ and centralizes $G_{0}$. Thus $\operatorname{ad}_{G_{-1}} F 1=$ $F \operatorname{Id}_{G_{-1}}$. Hence there is $s \in F$ such that

$$
\operatorname{ad}_{G_{-1}} t^{\prime}-\left(\operatorname{ad}_{G_{-1}} t^{\prime}\right)^{p}-\left(\operatorname{ad}_{G_{-1}} f\right)^{p}=\operatorname{ad}_{G_{-1}} s 1 .
$$

We now conclude from $(*)$ that

$$
\begin{aligned}
& \operatorname{ad}_{G_{-1}}\left(f-\left(\operatorname{ad} t^{\prime}\right)^{p-1}(f)+s 1\right) \\
& \quad=\operatorname{ad}_{G_{-1}}\left(f-\left(\operatorname{ad} t^{\prime}\right)^{p-1}(f)+t^{\prime}\right)-\left(\operatorname{ad}_{G_{-1}} t^{\prime}\right)^{p}-\left(\operatorname{ad}_{G_{-1}} f\right)^{p}=0 .
\end{aligned}
$$

As $\operatorname{ad}_{G_{-1}}\left(f-\left(\operatorname{ad} t^{\prime}\right)^{p-1}(f)+s 1\right) \in A(1 ; \underline{1}) \subset G_{0}$ and $G_{-1}$ is a faithful $G_{0^{-}}$ module, we obtain $f=\left(\operatorname{ad} t^{\prime}\right)^{p-1}(f)-s 1$. Set $g:=\left(\operatorname{ad} t^{\prime}\right)^{p-2}(f) \in A(1 ; \underline{1})$. Then $\exp \left(\operatorname{ad}_{G_{0}} g\right)$ is an inner automorphism of $G_{0}$, and

$$
\exp \left(\operatorname{ad}_{G_{0}} g\right)(t)=t+[g, t]=t-\left[t^{\prime}, g\right] \equiv t-f=t^{\prime} \quad \bmod (F 1) .
$$

This proves the first claim. The second claim is an immediate consequence.

In the notation of [3] the result 2) means that $I\left(G_{0}\right)=(0)$, or equivalently, $I(\tilde{H}) \subset A_{1}$. This proves the analogue of Corollary $\mathbf{7 . 2 . 8}$ of [3].

We fix $z \in C\left(G_{0}\right)$ such that $\operatorname{ad}_{G_{-1}} z=-\operatorname{Id}_{G_{-1}}$. Define $\gamma, \delta \in T^{*}$ by

$$
\begin{array}{cl}
\gamma(z)=0, & \gamma(T \cap K) \neq 0, \\
\delta(z)=-1, & \delta(T \cap K)=0 .
\end{array}
$$

Set $\Gamma_{i}:=\left\{\mu \in \Gamma \cup\{0\} \mid G_{i, \mu} \neq(0)\right\}$ and $m_{i}(\mu):=\operatorname{dim} G_{i, \mu}$. Note that

$$
\Gamma_{i} \subset-i \delta+G F(p) \gamma \quad \text { for all } i .
$$

We will now determine $T$ and $G_{-1}$ to some extent. In the following proposition we will use the notation of 3.1 and 3.2. Recall that $G_{-1}$ is a faithful irreducible $G_{0}$-module of dimension $<p^{2}-3 p$.

Proposition 3.3. 1) The ideal of $G_{0}$ generated by $T$ is $G_{0}$.

2) $T$ is mapped under an automorphism of $G_{0}$ in the respective cases of Proposition 3.1 onto

$$
\begin{aligned}
& F x \partial \oplus F z \quad \text { in cases } 2 \text { and } 4, \\
& F x \partial \oplus F z \quad \text { or } \quad F(1+x) \partial \oplus F z \quad \text { in case } 5 .
\end{aligned}
$$

3) In case 2 of Proposition 3.1 the following are true:

(a) $G_{0}$ has a subalgebra $Q$ such that

(i) $T \subset Q$,

(ii) $\operatorname{dim} G_{0} / Q=1$,

(iii) $Q^{(1)}$ acts nilpotently on $G$,

(iv) there is a root vector $w \in G_{-1}$ with $[Q, w] \subset F w$.

(b) (i) $\operatorname{dim} G_{-1}=p-1$ or $p, m_{-1}(\mu)=1 \forall \mu \in \Gamma_{-1}$, 
(ii) If $\operatorname{dim} G_{-1}=p-1$ then $\Gamma_{-1}=\delta+G F(p)^{*} \gamma$, If $\operatorname{dim} G_{-1}=p$ then $\Gamma_{-1}=\delta+G F(p) \gamma$.

In case 3 of Proposition 3.1 the following is true:

$$
\begin{gathered}
\Gamma_{-i}=i \delta+G F(p) \gamma \text { and } m_{-i}(i \delta+j \gamma)=m_{-i}(i \delta) \\
\text { for all } j \in G F(p) \text { and } i \not \equiv 0 \bmod (p) .
\end{gathered}
$$

In case 4 of Proposition 3.1 the following are true:

(a) $\left[G_{0} \cap W(1 ; \underline{1})_{(0)}, A(1 ; \underline{1})\right]$ acts nilpotently on $G$.

(b) $G_{0}$ has a subalgebra $Q$ such that

(i) $T \subset Q$,

(ii) $\operatorname{dim} G_{0} / Q=1$,

(iii) $Q^{(1)}$ acts nilpotently on $G$,

(iv) there is a root vector $w \in G_{-1}$ with $[Q, w] \subset F w$.

(c) $\operatorname{dim} G_{-1}=p$ and $m_{-i}(i \delta+j \gamma)=m_{-i}(i \delta)$, for all $j \in G F(p)$ and $i \not \equiv$ $0 \bmod (p)$.

In case 5 of Proposition 3.1 the following are true:

(a) $G_{0}=F x^{2} \partial \oplus F x \partial \oplus F \partial \oplus A(1 ; \underline{1})$.

(b) $\left[G_{0} \cap W(1 ; \underline{1})_{(0)}, A(1 ; \underline{1})\right]$ acts nilpotently on $G$.

(c) If $T=F x \partial \oplus F z$ then $G_{0}$ has a subalgebra $Q$ such that

(i) $T \subset Q$,

(ii) $\operatorname{dim} G_{0} / Q=1$,

(iii) $Q^{(1)}$ acts nilpotently on $G$,

(iv) there is a root vector $w \in G_{-1}$ with $[Q, w] \subset F w$.

(d) If $T=F(1+x) \partial \oplus F z$, then every eigenvector in $A(1 ; \underline{1})$ acts invertibly on $G_{-1}$.

(e) $\operatorname{dim} G_{-1}=p, m_{-i}(i \delta+j \gamma)=m_{-i}(i \delta)$ for all $j \in G F(p)$ and $i \not \equiv 0 \bmod (p)$.

Proof. (1) Let $\mathcal{J}$ be the ideal of $G_{0}$ generated by $T$. In all cases $G_{0} / \operatorname{rad} G_{0}$ is simple, and $T \not \subset \operatorname{rad} G_{0}$. Thus $G_{0}=\mathcal{J}+\operatorname{rad} G_{0}$. The result follows from Proposition 3.1 and a case-by-case analysis of the ideal structure.

(2) Decompose according to Lemma 3.2:

$$
G_{0}=K \oplus \operatorname{rad} G_{0}, \quad T=(T \cap K) \oplus F z .
$$

Due to Lemma 7.5.2(a), $\gamma$ is a proper root of $G$. Therefore, in cases 2) and 4) $T \cap K$ is conjugate by an inner automorphism to $F x \partial$. In case 5) we choose a generator $x$ of $A(1 ; \underline{1})$ such that $x$ or $(1+x)$ is an eigenvector with respect to $K \cap T$. Correspondingly, $T \cap K$ coincides with $F x \partial$ or with $F(1+x) \partial$.

(3) Case 2): For a description of the irreducible $W(1 ; \underline{1})$-modules we refer to [5]. They have dimension $p-1$ or $p^{k}(k>0)$. Thus in our case $\operatorname{dim} G_{-1}=p-1$ or $=p$. These modules have the property that $W(1 ; \underline{1})_{(0)}$ has a common eigenvector and $W(1 ; \underline{1})_{(1)}$ acts nilpotently. Set $Q:=W(1 ; \underline{1})_{(0)}+F z$. Then $T=F x \partial+F z \subset Q$. Thus (a)(i), (a)(ii), (a)(iv) are satisfied. Since $Q^{(1)} \subset W(1 ; \underline{1})_{(1)}$ acts nilpotently on $G_{-1}+G_{0}$, we have $\lambda\left(Q_{\mu}^{(1)}\right)=0$ for all $\lambda, \mu \in \Gamma$. Then $Q^{(1)}$ acts nilpotently on G.

If $\operatorname{dim} G_{-1}=p$ then $G_{-1}$ is induced by a 1-dimensional subrepresentation of $W(1 ; \underline{1})_{(0)}$. Then all roots $\delta+i \gamma$ occur with multiplicity 1 . If $\operatorname{dim} G_{-1}=p-1$ then $G_{-1} \cong A(1 ; \underline{1}) / F$ is the quotient of the $p$-dimensional module $A(1 ; \underline{1})$ by the 1 -dimensional submodule $F$. Then all roots $\delta+i \gamma$, except for one, say $i_{0}$, occur, 
each with multiplicity 1 . Here $\delta+i_{0} \gamma$ is the weight corresponding to the weight space $F$. Thus it is the 0 weight. Hence $0=\left(\delta+i_{0} \gamma\right)(T \cap K)=i_{0} \gamma(T \cap K)$, i.e., $i_{0}=0$.

Case 3): Choose a canonical basis $(e, h, f)$ for $K$ such that $T \cap K=F h$. There is a basis $(x, y, z)$ of the Heisenberg algebra $\mathcal{H}$ such that $x, y$ are eigenvectors with respect to $T, x \in G_{0, \ell \gamma}, y \in G_{0,-\ell \gamma}$. Note that $\ell \neq 0$, since otherwise $\tilde{H}$ would not act triangulably. If $i \not \equiv 0 \bmod (p)$ then $z$ acts on $G_{-i}$ as $-i \operatorname{Id} \neq 0$. Now every irreducible factor of a composition series of $G_{-i}$ as a $\mathcal{H}$-module is induced by a 1-dimensional subrepresentation of $F x+F z$, and hence every root occurs in every composition factor with multiplicity 1.

Cases 4), 5): It has been proved in [13, (III.2), p. 705] that $\operatorname{dim} G_{-1}=p$ and that $\left[G_{0} \cap W(1 ; \underline{1})_{(0)}, A(1 ; \underline{1})\right]$ acts nilpotently on $G_{-1}$.

In case 4$)$ set $Q:=W(1 ; \underline{1})_{(0)}+A(1 ; \underline{1})$. Then $T=F x \partial+F z \subset Q, \operatorname{dim} G_{0} / Q=1$. As $W(1 ; \underline{1})_{(1)}$ acts nilpotently on $G_{-1}+G_{0}$, so does $Q^{(1)}$. As in case 2$)$, this implies that $Q^{(1)}$ acts nilpotently on $G$. Clearly, as $Q^{(1)}$ acts nilpotently on $G_{-1}$, there is a common eigenvector for $Q$. To prove the assertions on the multiplicities we observe that $G_{0}$ contains the Heisenberg algebra $F \partial \oplus F x \oplus F 1$. The remaining part (c) is now proved similarly to the analoguous assertion of case 3 ).

We now consider case 5$)$. The only subalgebra isomorphic to $\mathfrak{s l}(2)$ containing $F x \partial$ is $F x^{2} \partial+F x \partial+F \partial$. Now suppose that $T=F(1+x) \partial \oplus F z$. Then there is $k \in \mathbb{N}$ such that $G_{0}=F(1+x)^{k+1} \partial \oplus F(1+x) \partial \oplus F(1+x)^{p-k+1} \partial \oplus A(1 ; \underline{1})$. We then adjust the generating element $x$ of $A(1 ; \underline{1})$ : Set $y:=(1+x)^{p-k}-1$. Then $((1+x) \partial)(1+y)=-k(1+x)^{p-k}$, whence $(1+x) \partial=-k(1+y) d / d y,(1+x)^{k+1} \partial=$ $-k d / d y,(1+x)^{p-k+1} \partial=-k(1+y)^{2} d / d y$. Hence

$$
G_{0}=F d / d y \oplus F(1+y) d / d y \oplus F(1+y)^{2} d / d y
$$

As the mapping $y^{i} \mapsto x^{i}$ defines an automorphism of $W(1 ; \underline{1})+A(1 ; \underline{1})$, this proves (a).

If $T=F x \partial+F z$, then we proceed as in case 4) to prove (c).

Suppose $T=F(1+x) \partial+F z$. The eigenspaces in $A(1 ; \underline{1})$ are of the form $F(1+x)^{k}, 0 \leq k \leq p-1$. Since $x \partial \in G_{0} \cap W(1 ; \underline{1})_{(0)}$, part (b) shows that (for $k \neq 0) x^{k} \in\left[G_{0} \cap W(1 ; \underline{1})_{(0)}, A(1 ; \underline{1})\right]$ acts nilpotently on $G_{-1}$. Then $(1+x)^{k}$ acts invertibly.

To prove (e) we consider the $T$-invariant Heisenberg algebra $F \partial \oplus F x \oplus F 1$ or $F \partial \oplus F(1+x) \oplus F 1$, respectively, and proceed as in case 3$)$.

Corollary 3.4. $A_{2} \neq(0)$.

Proof. Assume $A_{2}=(0)$. Theorem 2.7 yields the estimate

$$
p^{3}-p \leq \operatorname{dim} L \leq \operatorname{dim} A_{1}+\operatorname{dim} G_{0}+\operatorname{dim} A / A_{0}
$$

By assumption $A_{1}$ embeds injectively into $\operatorname{Hom}\left(G_{-1}, G_{0}\right)$; thus

$$
\operatorname{dim} A_{1} \leq\left(\operatorname{dim} G_{-1}\right)\left(\operatorname{dim} G_{0}\right) .
$$

According to (6.3.2) and (6.3.3) of $[3], A / A_{0}$ has at most $p^{2}-p+6$ roots, each of multiplicity at most 7 . Thus

$$
\begin{aligned}
p^{3}-p & \leq\left(\operatorname{dim} G_{-1}\right)\left(\operatorname{dim} G_{0}\right)+\operatorname{dim} G_{0}+7\left(p^{2}-p+6\right) \\
\operatorname{dim} G_{-1} & \leq 7 p
\end{aligned}
$$


We now check the possibilities of Proposition 3.1. In case 1) we obtain the contradiction (as $p>7$ )

$$
p^{3}-p \leq 28 p+4+7\left(p^{2}-p+6\right)=\left(7 p^{2}+22 p+46\right)-p<10 p^{2}-p .
$$

Case 3 ) is treated similarly.

To treat cases 2), 4) and 5) we observe that $\operatorname{dim} G_{-1} \leq p$ in these cases (see Proposition 3.3). Thus we obtain the contradiction

$$
p^{3}-p \leq p(2 p)+2 p+7\left(p^{2}-p+6\right) \leq 10 p^{2}-5 p .
$$

Recall that $M(G)$ is the maximal ideal of $G$ contained in $\sum_{i \leq-2} G_{i}$. This is a homogeneous ideal. Hence $G / M(G)$ is a graded algebra, and

$$
(G / M(G))_{i} \cong G_{i} \quad \text { for } i \geq-1 .
$$

Recall that $\Gamma_{P}$ denotes the set of proper roots. For the definition of $\Gamma_{E}$ and $\Gamma_{R}$ see $[3,(5.6 .5),(5.6 .7)]$.

Proposition 3.5. 1) $G / M(G)$ contains a unique minimal ideal $S$. $S$ is a simple graded subalgebra of $G / M(G)$ satisfying

$$
S_{i}=(G / M(G))_{i} \quad \text { for } i \leq-1 .
$$

2) If $\Gamma_{P}(A, T) \subset G F(p) \gamma$, then $S_{0} \cong G_{0}$.

3) $G_{1} \neq(0)$, and $G_{0}$ acts faithfully on $G_{1}$.

Proof. (1) According to Corollary 3.4 we have $A_{2} \neq(0)$, and hence $(G / M(G))_{2} \neq$ (0). Due to [23] $G / M(G)$ contains a unique minimal ideal $V$, which is graded by the grading of $G / M(G)$. Moreover, by [23] $(G / M(G))_{-1}=V_{-1}$, and hence $V_{1} \supset\left[(G / M(G))_{2}, V_{-1}\right] \neq(0)$. Therefore we are in the nondegenerate case of [23]. There is a simple graded algebra $S$ and $m \geq 0$ such that

$$
S_{i} \otimes A(m ; \underline{1})=V_{i}=V \cap(G / M(G))_{i} \quad \forall i,
$$

and

$$
V_{i}=(G / M(G))_{i} \quad \text { for } i \leq-1 .
$$

Suppose that $m \geq 1$. Then $G_{0}$ contains an ideal isomorphic to $V_{0}=S_{0} \otimes A(m ; \underline{1})$. The application of Proposition 3.1 in combination with a dimension argument yields first, that $m=1$ and $\operatorname{dim} S_{0} \leq 2$, and then that $V_{0}$ is solvable of dimension $\geq p$. This can only happen in cases 4) and 5) with $m=1$, $\operatorname{dim} S_{0}=1$. From Proposition 3.3 we conclude that $\operatorname{dim} G_{-1}=p$. Then $\operatorname{dim} S_{-1}=1$, whence

$$
\left[S_{-1} \otimes A(1 ; \underline{1}), S_{-1} \otimes A(1 ; \underline{1})\right]=\left[S_{-1}, S_{-1}\right] \otimes A(1 ; \underline{1})=(0) .
$$

Hence $\left((G / M(G))_{-1}\right)^{(1)}=(0)$. As $G_{-1}$ by definition generates $\sum_{i<0} G_{i}$, we obtain $G_{-2} \subset M(G)$.

As $\sum_{i \neq 0} V_{i}+\left[V_{-1},(G / M(G))_{1}\right]$ is an ideal of $G / M(G)$ and $V$ is minimal, we have

$$
\left[V_{-1},(G / M(G))_{1}\right]=V_{0} \cong A(1 ; \underline{1}) .
$$

In particular, there is a root $\tau \in \Gamma_{-1}$ such that $F 1=\left[V_{-1, \tau},(G / M(G))_{1,-\tau}\right]$. Then we have $\tau\left(\left[G_{-1, \tau}, G_{1,-\tau}\right]\right) \neq(0)$. According to Lemma 7.5.2 this forces $\tau \in$ $\Gamma_{P}(A, T)$. Lemma 6.1.1(c) shows that $\left|\Gamma_{-} \cap G F(p) \tau\right| \leq 6$. Due to Proposition 3.3 we have $G_{-i}=(0)$ or $i \tau \in \Gamma_{-i}$ for every $i$ satisfying $0<i<p$. Thus $G_{-7}=(0)$. 
Suppose that $A=A_{-1}$. Then Lemma 7.3.1 shows that $\left[G_{-1}, G_{1}\right]=G_{0}$, contradicting the fact that $\left[V_{-1},(G / M(G))_{1}\right] \subset V_{0} \neq(G / M(G))_{0}$ in the present cases. Thus $A \neq A_{-1}$. Then the proof of Lemma 7.6.1(1) (which works in our case without changes) yields that $G_{p-1} \neq(0)$, while we have shown above that $G_{-7}=(0)$. This contradiction proves the assertion.

(2) If $T \subset S_{0}$, then Proposition 3.3(1) yields the assertion. Thus, assume $T \not \subset S_{0}$. Since $S$ is simple, we have $\left[S_{-1}, S_{1}\right]=S_{0}([21,(3.3 .6)])$. Choose $\tau \in \Gamma_{-1}$ such that $\left[S_{-1, \tau}, S_{1,-\tau}\right] \neq(0)$. Due to the present assumption Lemma 7.5.2 shows that

$$
(0) \neq T \cap S_{0}=\left[S_{-1, \tau}, S_{1,-\tau}\right]=\operatorname{ker} \tau \text {. }
$$

Hence $M:=\sum_{i \in G F(p)} S_{i \tau}$ is a nilpotent subalgebra of $S$ which is selfnormalizing, as $\mu\left(T \cap S_{0}\right) \neq(0)$ for all $\mu \notin G F(p) \tau$. Thus $M$ is a CSA of $S$ which acts nontriangulably on $S$. As $S$ is simple, this cannot happen.

(3) As $A_{2} \neq(0)$ we have $G_{1} \neq(0)$. Set $J:=\left\{x \in G_{0} \mid\left[x, G_{1}\right]=(0)\right\}$ and suppose $J \neq(0)$. Then, as $z$ acts invertibly on $G_{1}, z \notin J$. As $J \neq(0)$ is an ideal of $G_{0}, G_{0}$ is necessarily of case 1) or 2) in Proposition 3.1 and $J=K$ is isomorphic to $\mathfrak{s l}(2)$ or $W(1 ; \underline{1})$ in the respective cases. Since $K$ annihilates $G_{1}$, we conclude that $G_{1} \subset G_{-\delta}$. According to the first part of this proposition there is an ideal $S$ of $G / M(G)$, which is simple as an algebra. The above yields

$$
S_{1} \subset S_{-\delta}, \quad S_{0}=\left[S_{-1}, S_{1}\right] .
$$

Since $S_{1}$ is a trivial $K$-module, $S_{0}$ is the sum of copies of the irreducible $K$-module $(G / M(G))_{-1}=S_{-1}$. In the present cases this is only possible if $S_{0}=K$. But then $\delta\left(T \cap S_{0}\right)=0$. Consequently $\sum_{i \in G F(p)} S_{i \delta}$ is a CSA of $S$ which is not triangulable. This contradiction shows $J=(0)$.

Lemma 7.4.1 is improved:

Lemma 3.6. Suppose that $G_{-i+1} \neq(0), G_{-i}=(0)$ for some $i, 2 \leq i \leq \frac{p+1}{2}$. Then

1) $M(G)=(0)$,

2) $\left|\Gamma_{-i+1}\right| \leq 4$,

3) $G_{0}=\mathfrak{s l}(2) \oplus F z$.

The proof is done in steps.

(1) Claim: Let $\lambda \in \Gamma_{-j}, j>0$, and $0 \neq w \in G_{-j, \lambda}$. Then there is $w^{\prime} \in G_{j,-\lambda}$ such that $\left[w, w^{\prime}\right] \neq 0$.

Proof. Let $c \in A_{-j, \lambda}$ satisfy $w=c+A_{-j+1}$. As $c \notin A_{0}$ and $A_{0}$ is a distinguished maximal subalgebra, we have $c \notin R_{\lambda}$, and hence there exists $d \in A_{-\lambda}$ with $[c, d] \notin I$. Note that $\lambda$ is of the form $\lambda=j \delta+k \gamma$. Therefore $-\lambda=-j \delta-k \gamma \in \bigcup_{\ell} \Gamma_{\ell}$, where $\ell \equiv j \bmod (p)$. As $G_{\frac{p+1}{2}}=(0)$ and hence $0<j<\frac{p+1}{2}$, we obtain that $d \in \bigcup_{\ell \equiv j, \ell>0} A_{\ell}=A_{j} \subset A_{0}$. As $[c, d]$ acts non-nilpotently, we have $d \notin A_{j+1}$. Put $w^{\prime}:=d+A_{j+1}$.

If $M(G) \neq(0)$ then we have $M(G) \cap G_{-i+1} \neq(0)$. The preceding step shows that this is impossible. Thus $M(G)=(0)$.

(2) Claim: Let $\lambda \in \Gamma_{-j}, j>0$, and $0 \neq w \in G_{-j, \lambda}$. Then for all but at most $1+\left|\Gamma_{-2 j}\right| \operatorname{roots} \tau \in \Gamma_{-j}$ we have $G_{-j, \tau} \subset\left[w, G_{0}\right]$. Consequently, $\left|\Gamma_{-j}\right| \leq$ $1+\left|\Gamma_{-2 j}\right|+\left|\Gamma_{0}\right|$. 
Proof. Choose $w^{\prime} \in G_{j,-\lambda}$ according to step (1). For any $\tau \in \Gamma_{-j}$ with $\tau\left(\left[w, w^{\prime}\right]\right) \neq$ 0 and every $e \in G_{-j, \tau}$ we have

$$
e \in F\left[\left[w, w^{\prime}\right], e\right] \subset F\left[w,\left[w^{\prime}, e\right]\right]+F\left[w^{\prime},[w, e]\right] \subset\left[w, G_{0}\right]+F\left[w^{\prime},[w, e]\right] .
$$

Now let $\tau \in \Gamma_{-j}$ be a root with $\tau\left(\left[w, w^{\prime}\right]\right)=0$. Put $\tau=:-j \delta+l \gamma$. If $\gamma\left(\left[w, w^{\prime}\right]\right)=0$ then $\delta\left(\left[w, w^{\prime}\right]\right)=0$ and $\left[w, w^{\prime}\right]=0$, contradicting the choice of $w^{\prime}$. Thus $\gamma\left(\left[w, w^{\prime}\right)\right] \neq$ 0 , and hence there is at most one root $\tau$ with this property. Thus the only possible exceptions are those $\tau$ for which $\tau\left(\left[w, w^{\prime}\right]\right)=0$ or $\tau+\lambda \in \Gamma_{-2 j}$. Clearly, $\left[w, G_{0}\right] \subset$ $\sum_{\mu \in \Gamma_{0}} G_{-j, \mu+\lambda}$.

We now prove the lemma:

If $G_{0}$ is as in case 1 ) of Proposition 3.1 then set $j=i-1$, whence $2 j>i-1$, i.e., $\Gamma_{-2 j}=\emptyset$. Step (2) yields $\left|\Gamma_{-i+1}\right| \leq 1+\left|\Gamma_{0}\right| \leq 4$.

In cases 2) and 4) of Proposition 3.1 the subalgebra $Q$ mentioned in Proposition 3.3 contains $T$ and has a common eigenvector $w$ in every $G_{-j}$. Set $j=i-1$. Then $G_{-i+1, \tau} \subset\left[w, G_{0}\right]+G_{-i+1, \tau_{0}}$ for suitable $\tau_{0}$. Set $G_{0}=Q+F \partial$ to obtain $\left[w, G_{0}\right]=[w, Q]+F[w, \partial]$, and hence $\left[w, G_{0}\right]$ carries only 2 roots. Thus $\left|\Gamma_{-i+1}\right| \leq 3<p-1$. Since $G_{-1}$ is a faithful irreducible $G_{0}$-module, it has at least $p-1$ roots. Thus $i \geq 3$. Next set $j=i-2$ and observe that $2 j \geq i-1$. Thus we obtain similarly $\left|\Gamma_{-i+2}\right| \leq 3<p-1$. In the present cases this implies that $G_{-i+1}$ and $G_{-i+2}$ are trivial as $G_{0}^{(1)}$-modules. Then, however, the trivial module $G_{-i+1}$ is as a quotient of $G_{-1} \otimes G_{-i+2}$ isomorphic to a sum of copies of $G_{-1}$ as $G_{0}^{(1)}$-modules. This is only possible if $G_{-i+1}=(0)$, a contradiction.

In case 3) we choose an arbitrary root vector $w$ and obtain as in case 1) that $\left|\Gamma_{-i+1}\right| \leq 1+\left|\Gamma_{0}\right| \leq 6<p$, contradicting Proposition 3.3.

Thus suppose that $G_{0}$ is as in case 5) of Proposition 3.1. As $M(G)=(0)$, Proposition 3.5 yields the existence a simple Lie algebra $S$, which is an ideal of $G$, such that $S_{i}=G_{i}$ for $i<0$. Lemma 7.3.1 shows that $S_{0}=\left[S_{-1}, G_{1}\right]=$ $\left[G_{-1}, G_{1}\right]=G_{0}$. The simplicity of $S$ implies that $S_{-i+1}$ is an irreducible $S_{0}$-module ([21, (3.3.5)]). Set $Q:=F x^{2} \partial+F x \partial+A(1 ; \underline{1})$ and recall (Proposition 3.3) that $Q^{(1)}$ acts nilpotently on $G_{-1}$, hence nilpotently on $\sum_{i<0} G_{i}$. Let $w \in G_{-i+1}$ be an eigenvector with respect to $Q$. We have, as $\left[w, G_{-j}\right]=(0)$ for $j>0$,

$$
\begin{gathered}
{\left[\left(\operatorname{ad} G_{1}\right)^{i-1}(w), G_{-i+1}\right] \subset \sum_{j=0}^{i-1}\left(\operatorname{ad} G_{1}\right)^{j}\left(\left[w,\left(\operatorname{ad} G_{1}\right)^{i-1-j}\left(G_{-i+1}\right)\right]\right)} \\
\subset \sum_{j=0}^{i-1}\left(\operatorname{ad} G_{1}\right)^{j}\left(\left[w, G_{-j}\right]\right) \subset\left[w, G_{0}\right] \subset F w+F[\partial, w] .
\end{gathered}
$$

On the other hand, $G_{-i+1}$ is an irreducible $G_{0}$-module [21, (3.3.5)], and as $z \in$ $A(1 ; \underline{1})$ acts as $(-i+1) \operatorname{Id} \neq 0$ then $\left\{(\operatorname{ad} \partial)^{k}(w), k=0, \ldots, p-1\right\}$ is a linearly independent set. We proved above that this set is mapped under $\left(\operatorname{ad} G_{1}\right)^{i-1}(w) \subset$ $G_{0}$ into $F w+F[\partial, w]$. In the present case 5) this is only possible if $\left(\operatorname{ad} G_{1}\right)^{i-1}(w) \subset$ $A(1 ; \underline{1})$. Since the space on the right hand side is invariant under $G_{0}$ and $G_{-i+1}=$ $S_{-i+1}$ is $G_{0}$-irreducible, this implies

$$
\left(\operatorname{ad} G_{1}\right)^{i-1}\left(G_{-i+1}\right) \subset A(1 ; \underline{1}) .
$$

Next set $U_{-1}:=\left(\operatorname{ad} G_{1}\right)^{i-2}\left(G_{-i+1}\right) \subset G_{-1}$. Then the above deliberations show that $\left[U_{-1}, G_{1}\right] \subset A(1 ; \underline{1}) \neq G_{0}$. According to an earlier remark this yields $U_{-1} \neq$ $G_{-1}$. As $G_{-1}$ is $G_{0}$-irreducible, we conclude that $U_{-1}=(0)$. 
Choose root vectors $u \in G_{-1, \lambda}, u^{\prime} \in G_{1,-\lambda}$ such that $\gamma\left(\left[u, u^{\prime}\right]\right) \neq 0$ (which is possible since $\left.\left[G_{-1}, G_{1}\right]=G_{0}\right)$ and set

$$
M:=F u \oplus F u^{\prime} \oplus F\left[u, u^{\prime}\right] .
$$

If $\lambda\left(\left[u, u^{\prime}\right]\right)=0$, then $M$ is a Heisenberg algebra. Since $\Gamma_{-i+1}=(i-1) \delta+G F(p) \gamma$, there is a root $\mu \in \Gamma_{-i+1}$ with $\mu\left(\left[u, u^{\prime}\right]\right) \neq 0$. The representation theory of the Heisenberg algebra shows that

$$
\left(\operatorname{ad} u^{\prime}\right)^{p-1}\left(G_{-i+1, \mu}\right) \neq(0)
$$

which contradicts the preceding result.

Thus $M \cong \mathfrak{s l}(2)$. Adjust $u, u^{\prime}$ so that

$$
\left[\left[u, u^{\prime}\right], u\right]=2 u, \quad\left[\left[u, u^{\prime}\right], u^{\prime}\right]=-2 u^{\prime} .
$$

By construction, $\gamma\left(\left[u, u^{\prime}\right]\right) \neq 0$. Thus $\left[u, u^{\prime}\right]$ has $p$ different eigenvalues on $G_{-i+1}$. The representation theory of $\mathfrak{s l}(2)$ shows that $\left(\operatorname{ad} u^{\prime}\right)^{p-1}\left(G_{-i+1}\right) \neq(0)$. This contradiction settles case 5) of Proposition 3.1.

We will now prove an analogue of Lemma 7.6.1 of [3].

Lemma 7.6.1 (revisited). There are $i<0, i \not \equiv 0 \bmod (p)$, and $j \in G F(p)$ such that

$$
m_{i}(-i \delta) \neq m_{i}(-i \delta+j \gamma)
$$

Proof. Suppose on the contrary that

$$
m_{i}(-i \delta)=m_{i}(-i \delta+j \gamma) \quad \forall i \not \equiv 0, \forall j \in G F(p) .
$$

The preceding lemma shows that $G_{-\frac{p+1}{2}} \neq(0)$. Thus $G F(p)^{*} \tau \subset \Gamma_{R}$ for every $\tau \notin G F(p) \gamma$, hence $\tau \notin \Gamma_{P}$. We obtain

$$
\gamma \in \Gamma_{P} \subset G F(p) \gamma
$$

Proposition 3.5(2) then applies and shows that $G / M(G)$ has a minimal ideal $S$, which is simple in its own right and $S_{0} \cong G_{0}$,

$$
S \triangleleft G / M(G), \quad S_{i}=(G / M(G))_{i} \text { for } i<0, \quad S_{0} \cong G_{0} .
$$

The simplicity of $S$ enforces $\left[S_{-1}, S_{1}\right]=S_{0} \cong G_{0}$, and hence $T$ is naturally embedded in $\left[S_{-1}, S_{1}\right]$. Choose $\eta \in \Gamma_{-1}$, such that $\gamma\left(\left[S_{-1, \eta}, S_{1,-\eta}\right]\right) \neq 0$. Since $\eta \notin G F(p) \gamma$ is improper, then Lemma 7.5.2(b) shows that $\eta\left(\left[S_{-1, \eta}, S_{1,-\eta}\right]\right)=0$. Thus there are $x \in S_{-1, \eta}, y \in S_{1,-\eta}$ such that

$$
\gamma([x, y])=1, \quad \eta([x, y])=0 .
$$

Observe that $\mathcal{H}:=F x \oplus F y \oplus F[x, y]$ is a Heisenberg algebra.

Next suppose that $G_{-p-1} \neq(0)$. Then, according to the present assumption, $G_{-p-1, \eta+j \gamma} \neq(0)$ for $1 \leq j \leq p-1$. Since $(\eta+j \gamma)([x, y])=j \gamma([x, y]) \neq 0$ for $j \neq 0$, the representation theory of the Heisenberg algebra yields that the $\mathcal{H}$-module generated by $G_{-p-1, \eta+j \gamma}$ must have nonzero intersection with $G_{-p, j \gamma}+G_{-2 p, j \gamma}$. So $m_{-}(j \gamma) \neq 0$ for all $j \neq 0$. Since $\gamma$ is proper this cannot happen. Consequently,

$$
G_{-p-1}=(0) .
$$

An analogous argument yields the estimate

$$
\operatorname{dim} G_{-1, \eta+j \gamma} \leq \operatorname{dim} G_{-p, j \gamma}+\operatorname{dim} G_{0, j \gamma} .
$$


Since $m_{-}(j \gamma) \neq 0$ only for at most 6 values of $j$, we have

$$
\operatorname{dim} G_{-p, j_{0} \gamma}=0 \text { for some } j_{0} \neq 0 .
$$

In the cases 1) and 2) of Proposition 3.1 we have $\operatorname{dim} G_{0, j \gamma} \leq 1$ for all $j \neq 0$. Consequently, $\operatorname{dim} G_{-1, \eta+j_{0} \gamma} \leq 1$. The general assumption of this lemma then shows that

$$
\operatorname{dim} G_{-1, \tau}=1 \quad \forall \tau \in \Gamma_{-1}
$$

in these cases. For all other cases Proposition 3.3 yields the same equality. As $G_{-p-1}=(0)$ we have $A_{\tau} \subset A_{-1}$, and then the above implies

$$
\operatorname{dim} A_{\tau} / A_{0} \cap A_{\tau}=1 \quad \forall \tau \in \Gamma_{-1} .
$$

Corollary 6.4.2(d) shows that all $\tau \in \Gamma_{-1}$ are non-Hamiltonian and non-BLOCK, and $(7.5 .2(\mathbf{c}))$ then gives us

$$
G_{\tau}=K_{\tau}(G) \quad \forall \tau \in \Gamma_{-1} .
$$

We are now going to apply earlier results to the simple algebra $S$. We have $S_{\tau}=K_{\tau}(S)$, and hence by definition $R K_{\tau}(S)=R_{\tau}(S)$ for $\tau \in \Gamma_{-1}$. Thus $\left[S_{-1, \tau}, S_{1,-\tau}\right] \neq(0)$ implies $\tau \in \Gamma_{E}(S, T)$. As $T$ is embedded in $\left[S_{-1}, S_{1}\right]$, then $\left|\Gamma_{-1} \cap \Gamma_{E}(S, T)\right| \geq 2$. Note that $(S, T)$ fulfils "Setting 1", and therefore Lemma 5.7.5 applies (although it is not clear whether or not $T$ is a torus of maximal dimension in a semisimple $p$-envelope of $S)$. As a consequence, $\Delta_{P}(S, T) \neq \Delta \backslash(0)$. This result contradicts Lemma $\mathbf{7 . 5 . 2 ( a )}$.

The proof of Lemma 7.7.1 works without changes. We even have simplifications, since we may assume that $G_{-\frac{p+1}{2}} \neq(0)$ and we know already that $G / M(G)$ has a minimal ideal which is simple in its own right.

Thus in the case of this section we have

$$
G_{0}=\mathfrak{s l}(2) \oplus F z, G_{1} \neq(0), G_{0} \text { acts faithfully on } G_{1} .
$$

A.A. Premet pointed out to me that in [3] presumably a wrong version of the recognition theorem for graded algebras has been used. Namely, some restricted Cartan type Lie algebras with the grading reverse to the standard grading satisfy all the assumptions of the recognition theorem for graded algebras ([3, Theorem 1.2.1]). So the statement in the conclusion that the Cartan type Lie algebras have standard grading seems to be incorrect.

The only proof which I know for the recognition theorem for filtered algebras needs the assumption that the associated graded algebra (if it is of Cartan type) carries the standard grading. In order to acchieve a complete proof of the classification it appearently is necessary not only in this note but also in [3] to ensure that the gradings of the Cartan type Lie algebras are standard.

Let $(e, f, h)$ denote an $\mathfrak{s l}(2)$-triple in $G_{0}$, so that $(e, f, h, z)$ is a basis of $G_{0}$. Define a $p$-mapping on $G_{0}$ by $e^{[p]}=0, h^{[p]}=h, f^{[p]}=0, z^{[p]}=z$. The definition of $z$ earlier in this section yields $\operatorname{ad}_{G_{-1}}\left(z^{[p]}\right)=\operatorname{ad}_{G_{-1}}(z)=\operatorname{ad}_{G_{-1}}(z)^{p}$. With this definition $G_{0}$ becomes a restricted Lie algebra which is the direct sum of the restricted ideals $G_{0}^{(1)}$ and $C\left(G_{0}\right)$.

One defines $G^{\prime}$ to be the algebra generated by $\sum_{i \leq 1} G_{i}$, and $N(G):=M\left(G^{\prime}\right)$. Clearly, $N(G)$ is a nilpotent ideal of $G^{\prime}$.

Lemma 3.7. Let $A$ be as in section 7 of [3] or as in section 3 of this note. Then the following are true. 
1) $G_{0}$ acts restrictedly on $G_{-1}$.

2) $N(G)=(0)$.

3) $G$ is classical or a Cartan type Lie algebra with standard grading.

Proof. (1) If $A$ is as in section 7 of [3] then $A$ is restricted, and hence $G_{0}$ acts restrictedly on $G_{-1}$.

Suppose $A$ is as in section 3 of this note. Let $\rho_{i}: G_{0} \rightarrow \mathfrak{g l}\left(G_{i}\right)$ denote the representation of $G_{0}$ on $G_{i}$ given by the multiplication in $G$. Let $\chi$ denote the character of the representation $\rho_{-1}$, i.e., $\rho_{-1}(u)^{p}-\rho_{-1}\left(u^{[p]}\right)=\chi(u)^{p} \operatorname{Id}_{G_{-1}}$ for all $u \in G_{0}$. Note that the $G_{0}$-module $G_{-i}$ is, for $i>0$, the homomorphic image of $\bigotimes^{i} G_{-1}\left(\right.$ as $\left.G_{-i}=\left[G_{-1}, \ldots\left[G_{-1}, G_{-1}\right] \ldots\right]\right)$ and hence every $\rho_{-i}$ has the character $i \chi$ (although in general it is not irreducible). According to Lemma 7.6.1(revisited) there are $i<0, i \not \equiv 0 \bmod (p)$, and $j \in G F(p)$ such that $m_{i}(-i \delta) \neq m_{i}(-i \delta+j \gamma)$. Then $\left.i \chi\right|_{G_{0}^{(1)}}=0$. As $\chi(z)=0$, this gives $\chi=0$. Therefore $G_{-1}$ is a restricted $G_{0}$-module.

We now have proved all conditions, so Kac' recognition theorem on graded Lie algebras (cf. $[3,(1.2 .1)]$ ) applies to $Q:=G^{\prime} / N(G)$. Thus $Q$ is isomorphic as a graded algebra to some $W(2 ; \underline{n}), K(3 ; \underline{n})$, or $H(2 ; \underline{n})^{(2)}+F\left(x_{1} D_{1}+x_{2} D_{2}\right) \subset Q \subset$ Der $H(2 ; \underline{n})^{(2)}$, or $Q$ is classical. Here the grading of the Cartan type Lie algebras is the standard or the reverse to the standard grading, while for the classical algebras the grading is the standard grading defined by a set of simple roots.

Suppose first that $Q$ is not classical. Then $Q$ contains a graded subalgebra $\tilde{Q} \in\left\{W(2 ; \underline{1}), K(3 ; \underline{1}), H(2 ; \underline{1})^{(2)}+F\left(x_{1} \partial_{1}+x_{2} \partial_{2}\right)\right\}$ in such a way that $Q_{-1}+Q_{0}+$ $Q_{1} \subset \tilde{Q}$. The arguments in [3, p. 206] show that $N(G)^{i} / N(G)^{i+1}$ is a trivial module for $\tilde{Q}^{(1)}$. Let $V$ be the inverse image of $\tilde{Q}^{(1)}$ in $G^{\prime}$. Then $\left[V, N(G)^{i}\right] \subset N(G)^{i+1}$ for all $i$, and hence

$$
\left[\bigcap_{n \geq 1} V^{(n)}, N(G)\right]=(0) .
$$

Note that $G_{0}^{(n)}=G_{0}^{(1)} \cong \mathfrak{s l}(2)$ for all $n>1$, and $\left[G_{-1}, G_{0}^{(1)}\right]=G_{-1}$. Also, in the cases under consideration we have $\left[\tilde{Q}_{0}^{(1)}, \tilde{Q}_{1}\right]=\tilde{Q}_{1}$. As $G_{0}+G_{1}$ maps under the homorphism $G^{\prime} \rightarrow G^{\prime} / N(G)$ isomorphically onto $\tilde{Q}_{0}+\tilde{Q}_{1}$, this means $\left[G_{0}^{(1)}, G_{1}\right]=G_{1}$. Then $G_{-1}, G_{1} \subset \bigcap_{n \geq 1} V^{(n)}$, and the above proves

$$
\left[G_{-1}, N(G)\right]=(0), \quad\left[\left[G_{-1}, G_{1}\right], N(G)\right]=(0) .
$$

If $\tilde{Q} \in\{W(2 ; \underline{1}), K(3 ; \underline{1})\}$, then $T \subset\left[G_{-1}, G_{1}\right]$. Thus $N(G) \subset C_{G}(T) \subset \sum_{i \geq 0} G_{i}$, whence $N(G)=(0)$.

Next suppose $\tilde{Q} \cong H(2 ; \underline{1})^{(2)}+F\left(x_{1} \partial_{1}+x_{2} \partial_{2}\right)$. Then $\left[G_{-1}, G_{1}\right]=G_{0}^{(1)}$, and there are independent roots $\alpha, \beta \in \Gamma_{-1}$, such that $-\alpha,-\beta \in \Gamma_{1},\left[G_{-1, \alpha}, G_{1,-\alpha}\right]=$ $T \cap G_{0}^{(1)},\left[G_{-1, \beta}, G_{1,-\beta}\right]=T \cap G_{0}^{(1)}, \alpha\left(T \cap G_{0}^{(1)}\right) \neq 0, \beta\left(T \cap G_{0}^{(1)}\right) \neq 0$. Lemma 7.5.2 implies that $\alpha, \beta$ are proper roots. Thus $\sum_{i<0}\left|\Gamma_{i}\right| \leq p^{2}-1-(2 p-2)+12<p^{2}-7$.

Since $T$ is mapped onto $F x_{1} \partial_{1}+F x_{2} \partial_{2}$ under the mapping $G^{\prime} \rightarrow G^{\prime} / N(G)$, it is easy to check that all root spaces (except the 0-root space) of $\tilde{Q}$ are 1-dimensional with respect to $T$. So if the grading of $Q$ is the reverse of the standard grading, then $\sum_{i<0}\left|\Gamma_{i}\right| \geq \sum_{i<0} \operatorname{dim} \tilde{Q}_{i}=p^{2}-7$. As this contradicts the previous estimate, the grading of $Q$ is the standard grading. Thus we have $Q_{-2}=(0)$. This means $G_{-2} \subset N(G)$, whence $G_{-3}=\left[G_{-1}, G_{-2}\right] \subset\left[G_{-1}, N(G)\right]=(0)$. Therefore Lemma 
7.3.1 applies and yields $\left[G_{-1}, G_{1}\right]=G_{0}$. But then $T \subset G_{0}$ acts trivially on $N(G)$, which is only possible if $N(G) \subset \sum_{i \geq 0} G_{i}$. Consequently, $N(G)=(0)$.

Suppose $Q$ is classical. The arguments in [3] give the result.

(3) We now have proved that the recognition theorem for graded algebras applies to $G$. Thus $G$ is isomorphic as a graded algebra to some $W(2 ; \underline{n}), K(3 ; \underline{n})$, or $H(2 ; \underline{n})^{(2)}+F\left(x_{1} D_{1}+x_{2} D_{2}\right) \subset G \subset \operatorname{Der} H(2 ; \underline{n})^{(2)}$ with the standard or the reverse to the standard grading, or $G$ is classical.

Suppose $G$ is of Cartan type with the grading reverse to the standard grading. Note that $T$ is the 2 -dimensional torus in $G_{0}$ (which also in this grading is the canonical 0 -component of $G$ ). If $G \cong W(2 ; \underline{n})$, or $G \cong K(3 ; \underline{n})$, or $H(2 ; \underline{n})^{(2)}+$ $F\left(x_{1} D_{1}+x_{2} D_{2}\right) \subset G \subset \operatorname{Der} H(2 ; \underline{n})^{(2)}$ with $\underline{n} \neq \underline{1}$, then it is immediate that $\sum_{i<0} G_{i}$ carries more than $p^{2}-p+6$ roots. If $H(2 ; \underline{1})^{(2)}+F\left(x_{1} D_{1}+x_{2} D_{2}\right) \subset$ $G \subset \operatorname{Der} H(2 ; \underline{1})^{(2)}$, then we derive the same conclusion by arguments given already in (2). As this contradicts the choice of $A_{0}$, the grading of $G$ is the standard grading.

The arguments given on page $227 / 228$ of [3] now apply to $A$ if $A$ is as in case (h) of Theorem 4.1.1 and if $G$ satisfies (9.3.1) or (9.3.2). As to the Lie algebras considered in this note, we are now able to prove that the case of this section will not occur at all.

Theorem 3.8. Let $L$ be simple satisfying $(A ; 3)$, and let $L_{p}$ be a semisimple $p$ envelope of L. Suppose that every maximal 2-dimensional torus $U \subset L_{p}$ has the property that $C_{L}(U)$ is a $C S A$ of $L$ and $U$ is contained in the p-envelope $\widetilde{C_{L}(U)}$ of $C_{L}(U)$ in $L_{p}$.

Let $T$ denote a maximal 2-dimensional torus having maximal number of proper roots, and $H:=C_{L}(T)$. Put $A:=L+\tilde{H}$, and let $A_{0}$ denote a maximal subalgebra of $A$ which contains $R(A)$. Then $\operatorname{rad} A_{0}$ acts nilpotently on $A$.

Proof. Suppose that $\operatorname{rad} A_{0}$ acts non-nilpotently on $A$. By assumption $A$ fulfils "Setting 2". Moreover, $T, A, A_{0}$ are as in the preceding deliberations. Consider the associated graded algebra $G$. Since $\operatorname{rad} A_{0}$ acts non-nilpotently, we have $\operatorname{rad} A_{0} \not \subset$ $A_{1}$, whence $\operatorname{rad} G_{0} \neq(0)$. Lemmas 7.6.1, 7.7.1, 3.7 prove that the assumptions of the recognition theorem on filtered algebras are satisfied. Thus $L$ is of classical or Cartan type. No classical algebra satisfies $(A ; 3)$. We also know that $L_{(0)} / L_{(1)}$ is a nonsolvable subalgebra of $\mathfrak{s l}(2) \oplus F z$. The recognition theorem then yields that $L$ is one of $W(2 ; \underline{n}), H(2 ; \underline{n} ; \Psi)^{(2)}, K(3 ; \underline{n} ; \Psi)^{(1)}$ with the canonical filtration. In particular, $T$ normalizes the unique proper subalgebra of maximal dimension of these Cartan type algebras.

Consider the algebra $W(2 ; \underline{n})$. This algebra contains the torus

$$
U=F x_{1} D_{1} \oplus F x_{2} D_{2} .
$$

Suppose $U$ is contained in a 3-dimensional torus $R$. Then $U \subset C_{L}(R)$, whence $C_{L}(R)$ acts non-nilpotently on $L$. This contradicts Theorem 1.5(1). Thus $U$ is a maximal torus. It is straightforward to check that

$$
\begin{aligned}
& C_{W(2 ; \underline{n})}\left(F x_{1} D_{1}\right) / \operatorname{rad} C_{W(2 ; \underline{n})}\left(F x_{1} D_{1}\right) \cong W\left(1 ; \underline{n_{2}}\right), \\
& C_{W(2 ; \underline{n})}\left(F x_{2} D_{2}\right) / \operatorname{rad} C_{W(2 ; \underline{n})}\left(F x_{2} D_{2}\right) \cong W\left(1 ; \underline{n_{1}}\right) .
\end{aligned}
$$

Theorem 2.2 now implies $n_{1}=n_{2}=1$. Then $L \cong W(2 ; \underline{1})$ has toral rank 2 , a contradiction. 
Consider the algebra $H(2 ; \underline{n} ; \Psi)^{(2)}$. Since $T$ normalizes $H(2 ; \underline{n} ; \Psi)^{(2)}{ }_{(0)}$, we have, up to conjugacy,

$$
T=F\left(x_{1} D_{1}+E_{1}\right) \oplus F\left(x_{2} D_{2}+E_{2}\right), \quad E_{i} \in W(2 ; \underline{n})_{(1)} .
$$

We now consider the associated graded algebra gr $(L+T)$. By definition $L$ satisfies the compatibility condition $H(2 ; \underline{n})^{(2)} \subset$ gr $L \subset H(2 ; \underline{n})$. Thus $H(2 ; \underline{n})^{(2)} \subset$ $\operatorname{gr}(L+T)$. Also, the above description of $T$ shows that $T$ is mapped as a torus on gr $L$ onto $F x_{1} D_{1} \oplus F x_{2} D_{2}$. Then $\operatorname{gr}(L+T)$ contains the 1-section

$$
F D_{1}+\sum_{i=1}^{p^{n_{1}}-1} F\left(x_{1}^{(i)} D_{1}-x_{1}^{(i-1)} x_{2} D_{2}\right)+T \text {. }
$$

The semisimple quotient of this 1 -section is isomorphic to $W\left(1 ; \underline{n_{1}}\right)$. Lifting this information to $L$, we obtain that there is a 1 -section $L\left(\alpha_{1}\right)$ such that $L\left(\alpha_{1}\right) / \operatorname{rad} L\left(\alpha_{1}\right)$ $\cong W\left(1 ; \underline{n_{1}}\right)$. As above, we conclude $n_{1}=n_{2}=1$. The algebras $H(2 ; \underline{1} ; \Psi)^{(2)}$ have toral rank $\leq 2$, as they are contained in $W(2 ; \underline{1})$.

We finally consider the algebra $K(3 ; \underline{n} ; \Psi)^{(1)}$. Since $m+3 \not \equiv 0 \bmod (p)$ for $m=3$, the algebra $K(3 ; \underline{n})$ is simple. The compatibility condition $K(3 ; \underline{n})^{(1)} \subset$ gr $K(3 ; \underline{n} ; \Psi)^{(1)} \subset K(3 ; \underline{n})$ shows that $K(3 ; \underline{n} ; \Psi)=K(3 ; \underline{n})$. The description of this algebra given in $[21, \S 4]$ uses the following Lie product on the divided power ring $A(3 ; \underline{n})$ :

$$
\begin{aligned}
& \left\langle x^{(a)}, x^{(b)}\right\rangle:=\left(\left(\begin{array}{c}
a+b-\varepsilon_{1}-\varepsilon_{2} \\
a-\varepsilon_{1}
\end{array}\right)-\left(\begin{array}{c}
a+b-\varepsilon_{1}-\varepsilon_{2} \\
a-\varepsilon_{2}
\end{array}\right)\right) x^{\left(a+b-\varepsilon_{1}-\varepsilon_{2}\right)} \\
& \quad+\left(\left(b_{1}+b_{2}+2 b_{3}-2\right)\left(\begin{array}{c}
a+b-\varepsilon_{3} \\
b
\end{array}\right)-\left(a_{1}+a_{2}+2 a_{3}-2\right)\left(\begin{array}{c}
\left.a+b-\varepsilon_{3}\right) \\
a
\end{array}\right)\right) x^{\left(a+b-\varepsilon_{3}\right)} .
\end{aligned}
$$

In particular,

$$
\begin{aligned}
& \left\langle x_{1} x_{2}, x^{(a)}\right\rangle=\left(a_{2}-a_{1}\right) x^{(a)}, \\
& \left\langle x_{3}, x^{(a)}\right\rangle=\left(a_{1}+a_{2}+2 a_{3}-2\right) x^{(a)} .
\end{aligned}
$$

Then $U:=F x_{1} x_{2} \oplus F x_{3}$ is a 2-dimensional torus which is contained in $K(3 ; \underline{n})$. As above, $U$ is a maximal torus. Theorem 2.2 provides a list of possibilities for the 1 -sections with respect to such a torus. We now compute 1 -sections of $K(3 ; \underline{n})$ with respect to $U$. Let $\alpha, \beta$ denote the roots defined by

$$
\alpha\left(x_{1} x_{2}\right)=1, \quad \alpha\left(x_{3}\right)=0, \quad \beta\left(x_{1} x_{2}\right)=0, \quad \beta\left(x_{3}\right)=1 .
$$

Then

$$
K(3 ; \underline{n})(\beta)=\operatorname{span}\left\{x^{(a)} \mid a_{1}-a_{2} \equiv 0 \bmod (p)\right\} .
$$

It is easy to check that

$$
K(3 ; \underline{n})(\beta) / \operatorname{rad}(K(3 ; \underline{n})(\beta)) \cong W\left(1 ; \underline{n_{3}}\right) .
$$

Thus Theorem 2.2 yields $n_{3}=1$. Next,

$$
K(3 ; \underline{n})(\alpha-\beta)=\operatorname{span}\left\{x^{(a)} \mid a_{2}+a_{3} \equiv 1 \bmod (p)\right\} .
$$

It can be checked that

$$
K(3 ; \underline{n})(\alpha-\beta) / \operatorname{rad}(K(3 ; \underline{n})(\alpha-\beta)) \cong W\left(1 ; \underline{n_{1}}\right) .
$$

Thus $n_{1}=1$. Similarly

$$
K(3 ; \underline{n})(-\alpha-\beta) / \operatorname{rad}(K(3 ; \underline{n})(-\alpha-\beta)) \cong W\left(1 ; \underline{n_{2}}\right)
$$

whence $n_{2}=1$. Therefore $L=K(3 ; \underline{1})$ is restricted. Then every torus of $L_{p}$ is contained in $L$, contradicting Theorem 1.5(1). 


\section{The CaSe $\operatorname{rad} G_{0}=(0)$}

Throughout this section we assume that $A$ satisfies "Setting 2 " and $T$ is an optimal torus. In contrast to [3] we now assume that $A_{0}$ is a distinguished subalgebra associated with $T$ of maximal dimension, and not just a distinguished maximal subalgebra. We do not see how section 8.10 of [3] could otherwise be salvaged. Let

$$
A=A_{-k} \supset \ldots \supset A_{0} \supset \ldots
$$

be a standard filtration. Let $G=\sum G_{i}$ be the associated graded algebra. In this section we assume that $G_{0}$ is semisimple. Let $\tilde{G}_{0}$ denote the $p$-envelope of $G_{0}$ in $\operatorname{Der} G_{0}$.

Proposition 4.1. 1) $\tilde{G}_{0}$ is semisimple, $T$ is naturally embedded in $\tilde{G}_{0}$, and $T R\left(G_{0}\right)$ $=2$.

2) The action of $G_{0}$ on $G_{-1}$ extends to a faithful restricted representation of $\tilde{G}_{0}$. 3) $C_{\tilde{G}_{0}}(T)$ is a CSA of $\tilde{G}_{0}$ which acts triangulably on $G$.

Proof. 1) Since $\operatorname{rad} \tilde{G}_{0} \supset\left[\operatorname{rad} \tilde{G}_{0}, \operatorname{ad} G_{0}\right]=\operatorname{ad}\left(\left(\operatorname{rad} \tilde{G}_{0}\right)\left(G_{0}\right)\right)$ is solvable, the ideal $\left(\operatorname{rad} \tilde{G}_{0}\right)\left(G_{0}\right)$ of $G_{0}$ is solvable. According to the assumption of this section this means $\left(\operatorname{rad} \tilde{G}_{0}\right)\left(G_{0}\right)=(0)$. As a subset of $\operatorname{Der} G_{0}$, now rad $\tilde{G}_{0}$ coincides with (0).

As $T \cap A_{1}=(0)$ there are embeddings $T \hookrightarrow G_{0} \hookrightarrow \operatorname{ad}_{G_{0}} G_{0} \subset \tilde{G}_{0}$. Consequently,

$$
2=\operatorname{dim} T \leq T R\left(G_{0}\right) \leq T R\left(A_{0}\right) \leq T R(L)=3 .
$$

Suppose that $T R\left(G_{0}\right)=3$. Let $R \subset \tilde{G}_{0}$ denote a 3 -dimensional torus of $\tilde{G}_{0}$. Then there is a 3 -dimensional torus $U$ in the $p$-envelope of $A_{0}$ in $L_{p}$ which is mapped onto $R[17,(1.1)]$. As $L$ satisfies $(A ; 3)$, then $C_{L}(U)$ acts nilpotently on $L$, and every 1-section $L(\mu)$ with respect to $U$ acts triangulably on $L$ (Theorem 1.5). Set $M:=\left(A_{0} \cap L+A_{1}\right) / A_{1} \subset G_{0}$. The above arguments imply that every 1-section $M(\mu)$ with respect to $R$ acts triangulably on $G$. Observe that $\operatorname{ad}_{G_{0}} M$ generates $\tilde{G}_{0}$ as a restricted algebra, and hence $T R(M)=3$.

Let

$$
S=\bigoplus_{i=1}^{r} S_{i} \otimes A\left(m_{i} ; \underline{1}\right)
$$

denote the socle of $M$, and $R_{i}$ the image of $R$ in $\operatorname{Der}\left(S_{i} \otimes A\left(m_{i} ; \underline{1}\right)\right)$ under the obvious homomorphisms. [17, (2.4)] applies with $K:=S_{i} \otimes A\left(m_{i} ; \underline{1}\right), T:=R_{i}$, and proves that every $S_{i} \otimes A\left(m_{i} ; \underline{1}\right)$ has a 2 -section with core $H(2 ; \underline{1} ; \Phi(\tau))^{(1)}$. Consequently, $T R\left(S_{i}\right)=T R\left(S_{i} \otimes A\left(m_{i} ; \underline{1}\right)\right) \geq T R\left(H(2 ; \underline{1} ; \Phi(\tau))^{(1)}\right)=2$, and

$$
3=T R(M) \geq \sum_{i=1}^{r} T R\left(S_{i} \otimes A\left(m_{i} ; \underline{1}\right)\right) \geq 2 r,
$$

whence $r=1$.

Let $S_{p}$ denote the $p$-envelope of $S$ in $\operatorname{Der} G_{0}$. [12, (1.3.(5))] yields for $I:=S$, $T:=R$ the estimate $\operatorname{dim} R \cap S_{p} \geq T R(S)$. As $T R(S) \geq \operatorname{dim} R \cap S_{p}$ by definition we obtain

$$
2 \leq \operatorname{dim} R \cap S_{p}=T R(S) \leq 3 .
$$


Suppose $T R(S)=2$. As $\operatorname{dim} R \cap S_{p}=2$ and $S$ is an ideal in $M$, there is a root $\alpha$ (possibly $\alpha=0$ ) with $M=S+M(\alpha)$ and $\alpha\left(R \cap S_{p}\right)=0$. Note that the $p$-envelope $\tilde{M}$ of $M$ in $\tilde{G}_{0}$ is of the form

$$
\begin{aligned}
\tilde{M} & =\operatorname{span}\left\{x^{[p]^{i}} \mid x \in \bigcup_{\mu \in \Gamma} M_{\mu}, i \geq 0\right\} \\
& =S_{p}+\operatorname{span}\left\{x^{[p]^{i}} \mid x \in \bigcup_{j \in G F(p)} M_{j \alpha}, i \geq 0\right\} .
\end{aligned}
$$

Therefore

$$
\begin{gathered}
C_{\tilde{M}}(R)=C_{S_{p}}(R)+\operatorname{span}\left\{x^{[p]^{i}} \mid x \in \bigcup_{j \in G F(p)^{*}} M_{j \alpha}, i>0\right\} \\
+\operatorname{span}\left\{x^{[p]^{i}} \mid x \in C_{M}(R), i \geq 0\right\} .
\end{gathered}
$$

$\alpha$ vanishes on $C_{M}(R)$ since $C_{M}(R)$ as a subalgebra of the image of $C_{L}(U)$ acts nilpotently, and $\alpha$ vanishes on $R \cap S_{p}$ as we have mentioned above. Now $C_{\tilde{M}}(R)^{(1)} \subset$ $\left(\sum_{\mu \in \Gamma} M(\mu)^{(1)}\right) \cap C_{M}(R)$ acts nilpotently on $M$ (since every 1-section acts triangulably) and hence every root is linear on $C_{\tilde{M}}(R)$. Then $\alpha$ vanishes on $C_{\tilde{M}}(R)$, proving $\alpha(R)=0$ and $\alpha=0$. Consequently, $M=S+C_{M}(R)$, and hence $R \subset \bigcap_{n \geq 0}\left(S+C_{M}(R)\right)^{[p]^{n}}=S_{p}$, which implies $T R(M)=T R(S)=2$. This is a contradiction.

Thus we have $T R(S)=3$ and $R \subset S_{p}$. Therefore $S_{1} \otimes F$ is simple and satisfies $(A ; 3)$. Theorem 2.7 shows that $\operatorname{dim} G_{-1} \geq p^{3}-p$. On the other hand, $G_{-1}$ has at most $p^{2}-p+6$ roots with respect to $T$, each of multiplicity $\leq 7<p$. This is a contradiction.

2) We consider $A \subset L_{p} \subset \operatorname{Der} A$. Let $N$ denote the $p$-envelope of $A_{0}$ in $L_{p}$. Since $A_{0}$ and $N$ respect the filtration, a homomorphic image $\bar{N}$ of $N$ is a $p$-envelope of $G_{0}$ in Der $G$. The $p$-mapping on $\bar{N}$ is defined by the $p$-mapping on $N$. By construction $\bar{N}$ acts restrictedly on $G_{-1}$.

Note that, since $C\left(\tilde{G}_{0}\right)=(0), \tilde{G}_{0}$ is a minimal $p$-envelope of $G_{0}$. According to $[21,(2.5 .8)]$ there exist an injective homomorphism

$$
\varphi: \tilde{G}_{0} \rightarrow \bar{N}, \varphi\left(\operatorname{ad}_{G_{0}} x\right)=\operatorname{ad}_{G} x \quad \forall x \in G_{0},
$$

and an ideal $J$ of $\bar{N}$ with $J \subset C(\bar{N})$, such that $\bar{N}=\varphi\left(\tilde{G}_{0}\right) \oplus J$.

Since $J$ centralizes $G_{0}$, and $G_{-1}$ is an irreducible $G_{0}$-module, we have that every element of $J$ acts as a multiple of the identity. Now if there is $x \in J$ with $\operatorname{ad}_{G_{-1}} x=\operatorname{Id}_{G-1}$, then $\bar{N}$ has a 3-dimensional torus $R$ such that $R \cap C(\bar{N}) \neq(0)$. Similarly to what we proved in 1$)$, we obtain $\tilde{G}_{0}=S_{p}$, where $S=S_{1} \otimes A(m ; \underline{1})$ and $S_{1} \cong H(2 ; \underline{1} ; \Phi(\tau))^{(1)}$ and the original torus $T$ acts on $S$ as a 2 -dimensional torus. But then $G_{-1}$ has $p^{2}-1$ roots with respect to $T$ (Lemma 2.6). This is a contradiction. Thus $J$ acts trivially on $G_{-1}$. Since $\tilde{G}_{0}$ is centerless, the isomorphism $\tilde{G}_{0} \cong \bar{N} / J$ is necessarily an isomorphism of restricted algebras. Therefore the action of $\tilde{G}_{0}$ on $G_{-1}$ is restricted.

Let $K$ denote an ideal of $\tilde{G}_{0}$ which annihilates $G_{-1}$. Since $G_{0}$ acts faithfully on $G_{-1}$ and $\left[K, G_{0}\right] \subset K \cap \tilde{G}_{0}^{(1)} \subset K \cap G_{0}$, we have that $\left[K, G_{0}\right]=(0)$. As $\tilde{G}_{0}$ is considered as a subalgebra of $\operatorname{Der} G_{0}$, this means $K=(0)$. 
3) As $T$ is a maximal torus of $\tilde{G}_{0}$, the centralizer $C_{\tilde{G}_{0}}(T)$ is nilpotent. This nilpotent algebra acts on every root space $G_{-1, \mu}$. The dimensions of these root spaces are bounded by $7<p$. Therefore every irreducible factor of $G_{-1, \mu}$ as a $C_{\tilde{G}_{0}}(T)$-module is 1-dimensional. Consequently, $C_{\tilde{G}_{0}}(T)^{(1)}$ acts nilpotently on $G_{-1}$. Observe that $T \subset \tilde{G}_{0}$ acts faithfully on $G_{-1}$. Therefore the roots on $G_{-1}$ span the full root lattice on $G$. As a result, $\mu\left(C_{\tilde{G}_{0}}(T)^{(1)}\right)=0$ for all roots $\mu$. This proves the proposition.

Lemma 4.2. No 1-section $\tilde{G}_{0}(\mu)$ with respect to $T$ is of Hamiltonian type.

Proof. Suppose that $\tilde{G}_{0}(\mu)$ is of Hamiltonian type. For arbitrary $\beta \in \Gamma_{-1}$ let

$$
\sum_{i \in G F(p)} G_{-1, \beta+i \mu}=V_{0} \supset V_{1} \supset \cdots \supset V_{t} \supset(0)
$$

denote a composition series as a $\tilde{G}_{0}(\mu)$-module. Let $\bar{V}_{i}:=V_{i} / V_{i+1}$ denote the simple quotient modules and $\rho_{i}: \tilde{G}_{0}(\mu) \rightarrow \mathfrak{g r}\left(\bar{V}_{i}\right)$ the associated representations. Note that $\bar{V}_{i}$ is a restricted $\tilde{G}_{0}(\mu)$-module. Moreover, as all root spaces $G_{-1, \gamma}$ have dimension $\leq 7<p$, then

$$
\operatorname{dim} \bar{V}_{i} \leq 7 p<p^{2}-2 .
$$

Since $\tilde{G}_{0}(\mu)$ acts faithfully on $G_{-1}$ and $\tilde{G}_{0}(\mu)$ is not solvable, there is a choice of $\beta$ and $i$ such that $M:=\rho_{i}\left(\tilde{G}_{0}(\mu)\right)$ is nonsolvable, and hence is of Hamiltonian type.

Recall that $C_{\tilde{G}_{0}(\mu)}(T)=C_{\tilde{G}_{0}}(T)$ is a CSA of toral rank 1 in $\tilde{G}_{0}(\mu)$ which acts triangulably on $G_{-1}$. Thus the assumptions of [13, section III] are satisfied. The result of $[13,($ III.3)] shows that $M$ cannot have an ideal $I \not \subset C(M)$. Then [13, (III, Corollary to Theorem 1)] yields that $\bigcap M^{(n)} / C\left(\bigcap M^{(n)}\right)$ is simple. By assumption $M$ is Hamiltonian. Then $H(2 ; \underline{1})^{(2)} \subset M / C(M) \subset H(2 ; \underline{1})$. If $C(M) \neq(0)$ then $C(M)=F \operatorname{Id}_{G_{-1}}$, and $[3,(7.2 .7)]$ shows that there is a root space $\bar{V}_{i, \gamma}$ of dimension $>7$, which is impossible. If $C(M)=(0)$ then $\bar{V}_{i}$ is a restricted nontrivial $H(2 ; \underline{1})^{(2)}$ module of dimension $<p^{2}-2$. Such modules do not exist $([8])$.

We are now going to apply arguments of section 8 of [3].

Proposition 4.3. $\tilde{G}_{0}$ is one of the following:

1) classical semisimple of rank 2 ;

2) $\mathfrak{s l}(2) \oplus W(1 ; \underline{1}), W(1 ; \underline{1}) \oplus W(1 ; \underline{1})$;

3) there are $S \in\{\mathfrak{s l}(2), W(1 ; \underline{1})\}$ and $\beta \in \Gamma_{0}$ such that

$$
\begin{aligned}
& S \otimes A(1 ; \underline{1}) \subset \tilde{G}_{0} \subset \operatorname{Der}(S \otimes A(1 ; \underline{1})), \\
& T \not \subset S \otimes A(1 ; \underline{1}), \\
& \tilde{G}_{0}=S \otimes A(1 ; \underline{1})+\tilde{G}_{0}(\beta) .
\end{aligned}
$$

Proof. Due to Proposition 4.1, $\tilde{G}_{0}$ is one of the algebras listed in Theorem 1.6. In addition, $\tilde{G}_{0}$ acts faithfully and restrictedly on $G_{-1}$, and $G_{-1}$ has at most $p^{2}-p+6$ different root spaces with respect to $T$, each of which has dimension at most 7 .

Cases 1), 2): As $\operatorname{TR}\left(\tilde{G}_{0}\right)=2$, these algebras do not occur.

Case 3): If $S_{1} \cong S_{2} \cong \mathfrak{s l}(2)$, then $\tilde{G}_{0}$ is classical. According to Lemma 4.2, neither $S_{1}$ nor $S_{2}$ is Hamiltonian.

Case 4$)$ is excluded by $[3,(8.4)]$. 
Case 5): The arguments of $[3,(8.10 .2)]$ apply in the present case and yield that $m=1, S \in\{\mathfrak{s l}(2), W(1 ; \underline{1})\}$. Then $T \cap(S \otimes A(1 ; \underline{1}))$ is nonzero, since otherwise $S \otimes A(1 ; \underline{1})$ would be solvable, and $T \not \subset S \otimes A(1 ; \underline{1})$ since $T R(S \otimes A(1 ; \underline{1}))=T R(S)=$ 1. Take a root $\beta$ satisfying $\beta(T \cap(S \otimes A(1 ; \underline{1})))=0$. We are now in the third case of this proposition.

Case 6$): S \cong W(1 ; \underline{2})$ : Since at least one root is proper and $\operatorname{dim} T=2$, then section $\mathrm{V}$ of $[14]$ shows that $T \cap W(1 ; \underline{2})$ is 1 -dimensional and restricted. The arguments of $[3,(8.8)]$ (which in fact do not need the assumption that every 2dimensional torus is standard) exclude this case.

$S \cong H(2 ;(2,1))^{(2)}:[3,(10.1 .1)]$ shows that there is a Hamiltonian 1-section, which is impossible.

$S \cong H(2 ; \underline{1} ; \Phi(\tau))^{(1)}$ is excluded by Lemma 2.6 .

$S \cong H(2 ; \underline{1} ; \Delta):[3,(2.1 .7)]$ yields $H(2 ; \underline{1} ; \Delta)_{p}=\operatorname{Der} H(2 ; \underline{1} ; \Delta)=H(2 ; 1 ; \Delta) \oplus$ $F\left(x_{1} \partial_{1}+x_{2} \partial_{2}\right)$. Since $\operatorname{dim} \operatorname{Der} H(2 ; \underline{1} ; \Delta) / H(2 ; \underline{1} ; \Delta)=1$ we have $T \cap H(2 ; \underline{1} ; \Delta) \neq$ (0). If $T \subset H(2 ; 1 ; \Delta)$, then, as $\left(x_{i} \partial_{i}\right)^{p}=x_{i} \partial_{i}$, Der $H(2 ; 1 ; \Delta)$ would contain a 3 -dimensional torus. As $\operatorname{Der} H(2 ; \underline{1} ; \Delta) \subset W(2 ; \underline{1})$, this is impossible. Again the arguments of $[3,(8.8),(8.9)]$ show that this algebra does not occur.

Case 7): The arguments of [3] given in the third paragraph of page 228 show that this case cannot occur.

Lemma 4.4. $G_{1} \neq(0)$.

Proof. According to Proposition 4.3 we have in cases 1) and 2) $\operatorname{dim} G_{0} \leq p^{2}$ and in case 3) $G_{0} \subset \operatorname{Der}(S \otimes A(1 ; \underline{1}))=(S \otimes A(1 ; \underline{1})) \oplus(F \operatorname{Id} \otimes W(1 ; \underline{1}))$ (as $S \in$ $\{\mathfrak{s l}(2), W(1 ; \underline{1})\})$. Then $\operatorname{dim} G_{0} \leq p^{2}+p$ holds, while Theorem 2.7 yields the estimate $\operatorname{dim} L \geq p^{3}-p$. As $\sum_{i<0} G_{i}$ has at most $p^{2}-p+6$ root spaces each of dimension at most 7 , we obtain

$$
\operatorname{dim} A_{1} \geq\left(p^{3}-p\right)-7\left(p^{2}-p+6\right)-\left(p^{2}+p\right) \geq(p-9) p^{2} .
$$

We now turn to the proof that case 3 of Proposition 4.3 is impossible. The following theorem describes the modules of semisimple Lie algebras.

Theorem 4.5 (cf. [20, (2.4)]). Let $M$ be a semisimple restricted Lie algebra and I a minimal nonabelian ideal of $M$. Suppose that $W$ is a finite dimensional restricted irreducible $M$-module with representation $\rho$ and assume that $\rho(I) \neq(0)$. Then there are a simple algebra $S, m \in \mathbb{N}$, and a $S$-module $U$ with representation $\bar{\rho}: U \rightarrow \mathfrak{g l}(U)$ such that

1. $I \cong S \otimes A(m ; \underline{1})$ under an algebra isomorphism $\psi_{1}$,

2. $W \cong U \otimes A(m ; \underline{1})$ under a vector space isomorphism $\psi_{2}$,

3. $\psi_{2}\left(\left(\left(\rho \circ \psi_{1}^{-1}\right)(y \otimes f)\right)\left(\psi_{2}^{-1}(u \otimes g)\right)\right)=\bar{\rho}(y)(u) \otimes$ fg for all $y \in S, u \in U$, $f, g \in A(m ; \underline{1})$.

Moreover, $\psi_{1}$ induces via the $\operatorname{ad}_{I}$-representation a restricted Lie algebra homomorphism $\tilde{\psi}_{1}: M \rightarrow((\operatorname{Der} S) \otimes A(m ; \underline{1})) \oplus(F \operatorname{Id} \otimes W(m ; \underline{1}))$. Let $\pi_{2}: M \rightarrow W(m ; \underline{1})$ denote the projection into the second summand. Then $\pi_{2}(M)$ is a transitive subalgebra of $W(m ; \underline{1})$. 
The action of $M$ on $W$ has the property

$$
\begin{aligned}
\left(\psi_{2} \circ \rho(D) \circ \psi_{2}^{-1}\right) & (u \otimes f) \\
= & (\operatorname{Id} \otimes f)\left(\left(\psi_{2} \circ \rho(D) \circ \psi_{2}^{-1}\right)(u \otimes 1)\right)+u \otimes \pi_{2}(D)(f)
\end{aligned}
$$

for all $D \in M, u \in U, f, \in A(m ; \underline{1})$.

In the proof of Lemma 8.10.3 BLOCK and WILSON show that $W \cong u\left(G_{0}\right) \otimes_{u(N)}$ $\tilde{W}_{1}$, where $N=(\mathfrak{s l}(2) \otimes A(1 ; \underline{1})+F \operatorname{Id} \otimes W(1 ; \underline{1})) \cap G_{0}$ (in the notation on p. 217 of [3]). They then claim that $W$ is uniquely determined, and from that statement they derive the form of $G_{-1}$ as a $G_{0}$-module. However, this claim might not be correct, since $N$ might have several nonequivalent faithful irreducible modules $\tilde{W}_{1}$ of dimension 2. This (probably false) claim is heavily used in the proofs of Lemmas 8.10.5 and 8.10.8. So some extra work seems to be necessary. In fact, it seems that one has to refine the construction. Namely, as was mentioned before, one has to consider a distinguished subalgebra $A_{0}$ of maximal dimension.

The preceding theorem clarifies the statement on the $G_{0}$-module $G_{-1}$. In order to salvage the statements in Lemmas 8.10.5 and 8.10.8 we present general results on Cartan prolongations (cf. [9]) and Spencer cohomology groups.

Proposition 4.6. Let $F$ be an arbitrary field of characteristic $p>0$. Consider $V:=U \otimes A(m ; \underline{1})$ as the natural module for a Lie subalgebra $M$ of $(\mathfrak{g l}(U) \otimes$ $A(m ; \underline{1})) \oplus(F \operatorname{Id} \otimes W(m ; \underline{1}))$. Set

$$
\begin{aligned}
\pi_{2}: & (\mathfrak{g l}(U) \otimes A(m ; \underline{1})) \oplus(F \operatorname{Id} \otimes W(m ; \underline{1})) \\
& \rightarrow(\mathfrak{g l}(U) \otimes A(m ; \underline{1})) \oplus(F \operatorname{Id} \otimes W(m ; \underline{1})) /(\mathfrak{g l}(U) \otimes A(m ; \underline{1})) \\
& \cong W(m ; \underline{1})
\end{aligned}
$$

the canonical homomorphism. Assume $\operatorname{dim} U>1$.

1. Let $\varphi: V \rightarrow M$ denote a linear mapping satisfying

$$
\varphi(v)\left(v^{\prime}\right)=\varphi\left(v^{\prime}\right)(v) \quad \text { for all } v, v^{\prime} \in V
$$

Then

$$
\left(\pi_{2} \circ \varphi\right)(u \otimes f)=f\left(\left(\pi_{2} \circ \varphi\right)(u \otimes 1)\right) \quad \text { for all } u \in U, f \in A(m ; \underline{1}) .
$$

2. Let $\Lambda: V \times V \rightarrow M$ denote a skewsymmetric bilinear mapping satisfying

$$
\Lambda\left(v, v^{\prime}\right)\left(v^{\prime \prime}\right)=\Lambda\left(v, v^{\prime \prime}\right)\left(v^{\prime}\right)+\Lambda\left(v^{\prime \prime}, v^{\prime}\right)(v) \quad \text { for all } v, v^{\prime}, v^{\prime \prime} \in V .
$$

Then

$$
\begin{aligned}
& \left(\left(\pi_{2} \circ \Lambda\right)\left(u \otimes f^{i-1}, u^{\prime} \otimes f\right)\right)(f) \\
& \quad=\left((i-2) f^{i}\left(\pi_{2} \circ \Lambda\right)\left(u \otimes 1, u^{\prime} \otimes 1\right)+(1-i) f^{i-1}\left(\pi_{2} \circ \Lambda\right)\left(u \otimes f, u^{\prime} \otimes 1\right)\right. \\
& \quad+(2-i) f^{i-1}\left(\pi_{2} \circ \Lambda\right)\left(u \otimes 1, u^{\prime} \otimes f\right) \\
& \quad+(i-1) f^{i-2}\left(\pi_{2} \circ \Lambda\right)\left(u \otimes f, u^{\prime} \otimes f\right) \\
& \left.\quad+f\left(\pi_{2} \circ \Lambda\right)\left(u \otimes f^{i-1}, u^{\prime} \otimes 1\right)\right)(f)
\end{aligned}
$$

for all linearly independent pairs $u, u^{\prime} \in U$, and all $f \in A(m ; \underline{1})$. 
Proof. (1) Set $\varphi_{2}:=\pi_{2} \circ \varphi$. Then $\varphi$ decomposes as $\varphi=F_{\varphi}+\operatorname{Id} \otimes \varphi_{2}$, where $F_{\varphi}: U \otimes A(m ; \underline{1}) \rightarrow \mathfrak{g l}(U) \otimes A(m ; \underline{1})$ is the associated mapping into the first summand. Clearly, the following equations are true:

$$
\begin{aligned}
\varphi(u \otimes f)\left(u^{\prime} \otimes g\right) & =g F_{\varphi}(u \otimes f)\left(u^{\prime} \otimes 1\right)+u^{\prime} \otimes \varphi_{2}(u \otimes f)(g), \\
\varphi(u \otimes f)\left(u^{\prime} \otimes 1\right) & =F_{\varphi}(u \otimes f)\left(u^{\prime} \otimes 1\right), \\
F_{\varphi}(u \otimes 1)\left(u^{\prime} \otimes 1\right) & =F_{\varphi}\left(u^{\prime} \otimes 1\right)(u \otimes 1) .
\end{aligned}
$$

From these equations we deduce

$$
\begin{aligned}
F_{\varphi}(u \otimes f)\left(u^{\prime} \otimes g\right) & =g F_{\varphi}(u \otimes f)\left(u^{\prime} \otimes 1\right) \\
& =g \varphi(u \otimes f)\left(u^{\prime} \otimes 1\right)=g \varphi\left(u^{\prime} \otimes 1\right)(u \otimes f) \\
& =f g F_{\varphi}\left(u^{\prime} \otimes 1\right)(u \otimes 1)+u \otimes g \varphi_{2}\left(u^{\prime} \otimes 1\right)(f) \\
& =f g F_{\varphi}(u \otimes 1)\left(u^{\prime} \otimes 1\right)+u \otimes g \varphi_{2}\left(u^{\prime} \otimes 1\right)(f) .
\end{aligned}
$$

An application of these results yields

$$
\begin{aligned}
\varphi(u \otimes f)\left(u^{\prime} \otimes g\right)= & F_{\varphi}(u \otimes f)\left(u^{\prime} \otimes g\right)+u^{\prime} \otimes \varphi_{2}(u \otimes f)(g) \\
= & f g F_{\varphi}(u \otimes 1)\left(u^{\prime} \otimes 1\right)+u \otimes g \varphi_{2}\left(u^{\prime} \otimes 1\right)(f) \\
& +u^{\prime} \otimes \varphi_{2}(u \otimes f)(g) .
\end{aligned}
$$

The left hand side coincides with $\varphi\left(u^{\prime} \otimes g\right)(u \otimes f)$, for which we have the analogous equation. Comparision of the right hand sides of these two equations yields

$$
u \otimes\left(g \varphi_{2}\left(u^{\prime} \otimes 1\right)-\varphi_{2}\left(u^{\prime} \otimes g\right)\right)(f)=u^{\prime} \otimes\left(f \varphi_{2}(u \otimes 1)-\varphi_{2}(u \otimes f)\right)(g)
$$

Since $\operatorname{dim} U>1$, one may choose $u, u^{\prime}$ linearly independent. The result follows.

(2) Set $\Lambda_{2}:=\pi_{2} \circ \Lambda$. Let $u, u^{\prime}, u^{\prime \prime} \in U$ and $P, P^{\prime}, P^{\prime \prime} \in A(m ; \underline{1})$ be arbitrary. Then

$$
\begin{aligned}
\Lambda(u \otimes & \left.P, u^{\prime} \otimes P^{\prime}\right)\left(u^{\prime \prime} \otimes P^{\prime \prime}\right) \\
= & \Lambda\left(u \otimes P, u^{\prime} \otimes P^{\prime}\right)\left(u^{\prime \prime} \otimes 1\right) P^{\prime \prime}+u^{\prime \prime} \otimes \Lambda_{2}\left(u \otimes P, u^{\prime} \otimes P^{\prime}\right)\left(P^{\prime \prime}\right) \\
= & \Lambda\left(u \otimes P, u^{\prime \prime} \otimes 1\right)\left(u^{\prime} \otimes P^{\prime}\right) P^{\prime \prime}+\Lambda\left(u^{\prime \prime} \otimes 1, u^{\prime} \otimes P^{\prime}\right)(u \otimes P) P^{\prime \prime} \\
& +u^{\prime \prime} \otimes \Lambda_{2}\left(u \otimes P, u^{\prime} \otimes P^{\prime}\right)\left(P^{\prime \prime}\right) \\
= & \Lambda\left(u \otimes P, u^{\prime \prime} \otimes 1\right)\left(u^{\prime} \otimes 1\right) P^{\prime} P^{\prime \prime}+u^{\prime} \otimes P^{\prime \prime} \Lambda_{2}\left(u \otimes P, u^{\prime \prime} \otimes 1\right)\left(P^{\prime}\right) \\
& +\Lambda\left(u^{\prime \prime} \otimes 1, u^{\prime} \otimes P^{\prime}\right)(u \otimes 1) P P^{\prime \prime}+u \otimes P^{\prime \prime} \Lambda_{2}\left(u^{\prime \prime} \otimes 1, u^{\prime} \otimes P^{\prime}\right)(P) \\
& +u^{\prime \prime} \otimes \Lambda_{2}\left(u \otimes P, u^{\prime} \otimes P^{\prime}\right)\left(P^{\prime \prime}\right) .
\end{aligned}
$$


Taking into account the cyclic symmetry, one obtains

$$
\begin{aligned}
& 0=\Lambda\left(u \otimes P, u^{\prime} \otimes P^{\prime}\right)\left(u^{\prime \prime} \otimes P^{\prime \prime}\right)+\Lambda\left(u^{\prime} \otimes P^{\prime}, u^{\prime \prime} \otimes P^{\prime \prime}\right)(u \otimes P) \\
& +\Lambda\left(u^{\prime \prime} \otimes P^{\prime \prime}, u \otimes P\right)\left(u^{\prime} \otimes P^{\prime}\right) \\
& =\Lambda\left(u \otimes P, u^{\prime \prime} \otimes 1\right)\left(u^{\prime} \otimes 1\right) P^{\prime} P^{\prime \prime}+\Lambda\left(u^{\prime} \otimes P^{\prime}, u \otimes 1\right)\left(u^{\prime \prime} \otimes 1\right) P P^{\prime \prime} \\
& +\Lambda\left(u^{\prime \prime} \otimes P^{\prime \prime}, u^{\prime} \otimes 1\right)(u \otimes 1) P P^{\prime} \\
& +u^{\prime} \otimes P^{\prime \prime} \Lambda_{2}\left(u \otimes P, u^{\prime \prime} \otimes 1\right)\left(P^{\prime}\right)+u^{\prime \prime} \otimes P \Lambda_{2}\left(u^{\prime} \otimes P^{\prime}, u \otimes 1\right)\left(P^{\prime \prime}\right) \\
& +u \otimes P^{\prime} \Lambda_{2}\left(u^{\prime \prime} \otimes P^{\prime \prime}, u^{\prime} \otimes 1\right)(P) \\
& +\Lambda\left(u^{\prime \prime} \otimes 1, u^{\prime} \otimes P^{\prime}\right)(u \otimes 1) P P^{\prime \prime}+\Lambda\left(u \otimes 1, u^{\prime \prime} \otimes P^{\prime \prime}\right)\left(u^{\prime} \otimes 1\right) P P^{\prime} \\
& +\Lambda\left(u^{\prime} \otimes 1, u \otimes P\right)\left(u^{\prime \prime} \otimes 1\right) P^{\prime} P^{\prime \prime} \\
& +u \otimes P^{\prime \prime} \Lambda_{2}\left(u^{\prime \prime} \otimes 1, u^{\prime} \otimes P^{\prime}\right)(P)+u^{\prime} \otimes P \Lambda_{2}\left(u \otimes 1, u^{\prime \prime} \otimes P^{\prime \prime}\right)\left(P^{\prime}\right) \\
& +u^{\prime \prime} \otimes P^{\prime} \Lambda_{2}\left(u^{\prime} \otimes 1, u \otimes P\right)\left(P^{\prime \prime}\right) \\
& +u^{\prime \prime} \otimes \Lambda_{2}\left(u \otimes P, u^{\prime} \otimes P^{\prime}\right)\left(P^{\prime \prime}\right)+u \otimes \Lambda_{2}\left(u^{\prime} \otimes P^{\prime}, u^{\prime \prime} \otimes P^{\prime \prime}\right)(P) \\
& +u^{\prime} \otimes \Lambda_{2}\left(u^{\prime \prime} \otimes P^{\prime \prime}, u \otimes P\right)\left(P^{\prime}\right) \\
& =\Lambda\left(u^{\prime} \otimes 1, u^{\prime \prime} \otimes 1\right)(u \otimes P) P^{\prime} P^{\prime \prime}+\Lambda\left(u^{\prime \prime} \otimes 1, u \otimes 1\right)\left(u^{\prime} \otimes P^{\prime}\right) P P^{\prime \prime} \\
& +\Lambda\left(u \otimes 1, u^{\prime} \otimes 1\right)\left(u^{\prime \prime} \otimes P^{\prime \prime}\right) P P^{\prime} \\
& +u^{\prime} \otimes P^{\prime \prime} \Lambda_{2}\left(u \otimes P, u^{\prime \prime} \otimes 1\right)\left(P^{\prime}\right)+u^{\prime \prime} \otimes P \Lambda_{2}\left(u^{\prime} \otimes P^{\prime}, u \otimes 1\right)\left(P^{\prime \prime}\right) \\
& +u \otimes P^{\prime} \Lambda_{2}\left(u^{\prime \prime} \otimes P^{\prime \prime}, u^{\prime} \otimes 1\right)(P) \\
& +u \otimes P^{\prime \prime} \Lambda_{2}\left(u^{\prime \prime} \otimes 1, u^{\prime} \otimes P^{\prime}\right)(P)+u^{\prime} \otimes P \Lambda_{2}\left(u \otimes 1, u^{\prime \prime} \otimes P^{\prime \prime}\right)\left(P^{\prime}\right) \\
& +u^{\prime \prime} \otimes P^{\prime} \Lambda_{2}\left(u^{\prime} \otimes 1, u \otimes P\right)\left(P^{\prime \prime}\right) \\
& +u^{\prime \prime} \otimes \Lambda_{2}\left(u \otimes P, u^{\prime} \otimes P^{\prime}\right)\left(P^{\prime \prime}\right)+u \otimes \Lambda_{2}\left(u^{\prime} \otimes P^{\prime}, u^{\prime \prime} \otimes P^{\prime \prime}\right)(P) \\
& +u^{\prime} \otimes \Lambda_{2}\left(u^{\prime \prime} \otimes P^{\prime \prime}, u \otimes P\right)\left(P^{\prime}\right) \\
& =\Lambda\left(u^{\prime} \otimes 1, u^{\prime \prime} \otimes 1\right)(u \otimes 1) P P^{\prime} P^{\prime \prime}+u \otimes P^{\prime} P^{\prime \prime} \Lambda_{2}\left(u^{\prime} \otimes 1, u^{\prime \prime} \otimes 1\right)(P) \\
& +\Lambda\left(u^{\prime \prime} \otimes 1, u \otimes 1\right)\left(u^{\prime} \otimes 1\right) P P^{\prime} P^{\prime \prime}+u^{\prime} \otimes P P^{\prime \prime} \Lambda_{2}\left(u^{\prime \prime} \otimes 1, u \otimes 1\right)\left(P^{\prime}\right) \\
& +\Lambda\left(u \otimes 1, u^{\prime} \otimes 1\right)\left(u^{\prime \prime} \otimes 1\right) P P^{\prime} P^{\prime \prime}+u^{\prime \prime} \otimes P P^{\prime} \Lambda_{2}\left(u \otimes 1, u^{\prime} \otimes 1\right)\left(P^{\prime \prime}\right) \\
& +u^{\prime} \otimes P^{\prime \prime} \Lambda_{2}\left(u \otimes P, u^{\prime \prime} \otimes 1\right)\left(P^{\prime}\right)+u^{\prime \prime} \otimes P \Lambda_{2}\left(u^{\prime} \otimes P^{\prime}, u \otimes 1\right)\left(P^{\prime \prime}\right) \\
& +u \otimes P^{\prime} \Lambda_{2}\left(u^{\prime \prime} \otimes P^{\prime \prime}, u^{\prime} \otimes 1\right)(P) \\
& +u \otimes P^{\prime \prime} \Lambda_{2}\left(u^{\prime \prime} \otimes 1, u^{\prime} \otimes P^{\prime}\right)(P)+u^{\prime} \otimes P \Lambda_{2}\left(u \otimes 1, u^{\prime \prime} \otimes P^{\prime \prime}\right)\left(P^{\prime}\right) \\
& +u^{\prime \prime} \otimes P^{\prime} \Lambda_{2}\left(u^{\prime} \otimes 1, u \otimes P\right)\left(P^{\prime \prime}\right) \\
& +u^{\prime \prime} \otimes \Lambda_{2}\left(u \otimes P, u^{\prime} \otimes P^{\prime}\right)\left(P^{\prime \prime}\right)+u \otimes \Lambda_{2}\left(u^{\prime} \otimes P^{\prime}, u^{\prime \prime} \otimes P^{\prime \prime}\right)(P) \\
& +u^{\prime} \otimes \Lambda_{2}\left(u^{\prime \prime} \otimes P^{\prime \prime}, u \otimes P\right)\left(P^{\prime}\right) \\
& =0+u \otimes\left(P^{\prime} P^{\prime \prime} \Lambda_{2}\left(u^{\prime} \otimes 1, u^{\prime \prime} \otimes 1\right)+P^{\prime} \Lambda_{2}\left(u^{\prime \prime} \otimes P^{\prime \prime}, u^{\prime} \otimes 1\right)\right. \\
& \left.+P^{\prime \prime} \Lambda_{2}\left(u^{\prime \prime} \otimes 1, u^{\prime} \otimes P^{\prime}\right)+\Lambda_{2}\left(u^{\prime} \otimes P^{\prime}, u^{\prime \prime} \otimes P^{\prime \prime}\right)\right)(P)
\end{aligned}
$$




$$
\begin{aligned}
& +u^{\prime} \otimes\left(P P^{\prime \prime} \Lambda_{2}\left(u^{\prime \prime} \otimes 1, u \otimes 1\right)+P^{\prime \prime} \Lambda_{2}\left(u \otimes P, u^{\prime \prime} \otimes 1\right)\right. \\
& \left.+P \Lambda_{2}\left(u \otimes 1, u^{\prime \prime} \otimes P^{\prime \prime}\right)+\Lambda_{2}\left(u^{\prime \prime} \otimes P^{\prime \prime}, u \otimes P\right)\right)\left(P^{\prime}\right) \\
& +u^{\prime \prime} \otimes\left(P P^{\prime} \Lambda_{2}\left(u \otimes 1, u^{\prime} \otimes 1\right)+P \Lambda_{2}\left(u^{\prime} \otimes P^{\prime}, u \otimes 1\right)\right. \\
& \left.+P^{\prime} \Lambda_{2}\left(u^{\prime} \otimes 1, u \otimes P\right)+\Lambda_{2}\left(u \otimes P, u^{\prime} \otimes P^{\prime}\right)\right)\left(P^{\prime \prime}\right) .
\end{aligned}
$$

Now set $u^{\prime \prime}=u$ and $u, u^{\prime}$ linearly independent, $P=P^{\prime}=f, P^{\prime \prime}=f^{i-1}$. Then, as $D\left(f^{i-1}\right)=(i-1) f^{i-2} D(f)$ for all $D \in W(m ; \underline{1})$,

$$
\begin{aligned}
0= & \left(f^{i} \Lambda_{2}\left(u^{\prime} \otimes 1, u \otimes 1\right)+f \Lambda_{2}\left(u \otimes f^{i-1}, u^{\prime} \otimes 1\right)\right. \\
& \left.+f^{i-1} \Lambda_{2}\left(u \otimes 1, u^{\prime} \otimes f\right)+\Lambda_{2}\left(u^{\prime} \otimes f, u \otimes f^{i-1}\right)\right)(f) \\
& +(i-1) f^{i-2}\left(f^{2} \Lambda_{2}\left(u \otimes 1, u^{\prime} \otimes 1\right)+f \Lambda_{2}\left(u^{\prime} \otimes f, u \otimes 1\right)\right. \\
& \left.+f \Lambda_{2}\left(u^{\prime} \otimes 1, u \otimes f\right)+\Lambda_{2}\left(u \otimes f, u^{\prime} \otimes f\right)\right)(f) \\
= & \left(-f^{i} \Lambda_{2}\left(u \otimes 1, u^{\prime} \otimes 1\right)+f \Lambda_{2}\left(u \otimes f^{i-1}, u^{\prime} \otimes 1\right)\right. \\
& +f^{i-1} \Lambda_{2}\left(u \otimes 1, u^{\prime} \otimes f\right)-\Lambda_{2}\left(u \otimes f^{i-1}, u^{\prime} \otimes f\right) \\
& +(i-1) f^{i} \Lambda_{2}\left(u \otimes 1, u^{\prime} \otimes 1\right)-(i-1) f^{i-1} \Lambda_{2}\left(u \otimes 1, u^{\prime} \otimes f\right) \\
& \left.-(i-1) f^{i-1} \Lambda_{2}\left(u \otimes f, u^{\prime} \otimes 1\right)+(i-1) f^{i-2} \Lambda_{2}\left(u \otimes f, u^{\prime} \otimes f\right)\right)(f) .
\end{aligned}
$$

This gives the result.

In the following we assume that $G$ is as in section 8 of [3] or as in section 4 of this note. The arguments in the proofs of Lemmas 8.10.1, 8.10.2, 8.10.4, 8.10.6 need no changes at all. In Lemma 8.10.3 the structure of the $G_{0}$-module $G_{-1}$ is now determined by an application of Theorem 4.5. In particular, $A$ is as in Lemma 8.10.3. If $L$ satisfies $(A ; 3)$, then Lemma 4.4 shows that $G_{1} \neq(0)$. So the arguments presented in the proof of Lemma 8.10.3 apply and yield a proof of the statements of this lemma; however, we have to keep in mind that $G_{-1}$ might be not the canonical $G_{0}$-module.

Lemma 8.10.5 (revisited). Let $A$ and $G$ be as in Lemma 8.10.3. Suppose

$$
G_{0} /(\mathfrak{s l}(2) \otimes A(1 ; \underline{1})) \cong W(1 ; \underline{1}) .
$$

Then $\alpha_{2} \in \Delta_{P}(A, T)$ and $G_{-2}=(0)$.

The proof remains unchanged. However, it seems that the proof is not sufficient for the additional statement " $\left[G_{-1}, G_{1}\right] \subset \mathfrak{s l}(2) \otimes A(1 ; \underline{1})$ " in the original version of the lemma.

Lemma 4.7. Let $A$ and $G$ be as in Lemma 8.10.3. Then $T \subset(\mathfrak{s l}(2) \otimes A(1 ; \underline{1})) \oplus$ $\left(F \operatorname{Id} \otimes W(1 ; \underline{1})_{(0)}\right)$. 
Proof. 1) Suppose $T \not \subset(\mathfrak{s l}(2) \otimes A(1 ; \underline{1})) \oplus\left(F \operatorname{Id} \otimes W(1 ; \underline{1})_{(0)}\right)$. Lemma 8.10.5 shows that $G_{0} / \mathfrak{s l}(2) \otimes A(1 ; \underline{1}) \approx W(1 ; \underline{1})$. Then we are in the situation of Lemma 8.10.6. Hence $\left[G_{-1}, G_{1}\right] \subset \mathfrak{s l}(2) \otimes A(1 ; \underline{1})$ and $G_{-2}=(0)$.

According to Theorem 4.5 we write $G_{-1} \cong U \otimes A(1 ; \underline{1})$, where $U$ is the 2 dimensional irreducible $\mathfrak{s l}(2)$-module.

2) Choose $T$-invariant vector spaces

$$
V_{-1} \subset \sum_{j \in \mathbb{F}_{p}} A_{ \pm \alpha / 2+j \alpha_{2}}, \quad V_{0}^{\prime} \subset V_{0} \subset \sum_{j \in \mathbb{F}_{p}} A_{ \pm \alpha+j \alpha_{2}}+\sum_{j \in \mathbb{F}_{p}} A_{j \alpha_{2}}
$$

such that

$$
A=V_{-1} \oplus A_{0}, \quad A_{0}=V_{0} \oplus A_{1}, \quad V_{0}^{\prime}+A_{1} / A_{1}=\mathfrak{s l}(2) \otimes A(1 ; \underline{1}) .
$$

Considering the associated graded Lie algebra $G$ and the root space decomposition of $A$, one concludes that

$$
\left[V_{-1}, V_{-1}\right] \subset A_{0}, \quad\left[V_{-1}, V_{0}\right] \subset V_{-1}+A_{1}, \quad\left[V_{-1}, A_{1}\right] \subset V_{0}^{\prime}+A_{1},
$$

and $\left[V_{0}^{\prime}, V_{0}\right] \subset V_{0}^{\prime}+A_{1}$. Thus

$$
J:=A_{1}+V_{0}^{\prime}+V_{-1}+\left[V_{-1}, V_{-1}\right]
$$

is a nonzero ideal of $A$. If $A$ is as in [3] then it satisfies (7.0.1). If $L$ satisfies $(A ; 3)$ then it is ruled by "Setting 2 ". In both cases $H \cap J$ generates the full torus $T$ under the $p$-mapping.

As $T \not \subset \mathfrak{s l}(2) \otimes A(1 ; \underline{1})$ we have $V_{0}^{\prime} \cap H \neq H$. Thus

$$
\left[V_{-1}, V_{-1}\right] \not \subset V_{0}^{\prime} \text {. }
$$

3) Let $\sigma_{-1}: G_{-1} \rightarrow V_{-1}$ denote the linear isomorphism given by the property $\sigma_{-1}(v)+A_{0} / A_{0}=v$ for all $v \in G_{-1}$. The multiplication on $A$ gives rise to a skewsymmetric bilinear mapping

$$
\Lambda: G_{-1} \times G_{-1} \rightarrow G_{0}, \quad \Lambda\left(v, v^{\prime}\right):=\left[\sigma_{-1}(v), \sigma_{-1}\left(v^{\prime}\right)\right]+A_{1} .
$$

Since

$$
\begin{aligned}
{\left[\Lambda\left(v, v^{\prime}\right), v^{\prime \prime}\right] } & =\left[\left[\sigma_{-1}(v), \sigma_{-1}\left(v^{\prime}\right)\right]+A_{1}, \sigma_{-1}\left(v^{\prime \prime}\right)+A_{0}\right] \\
& =\left[\left[\sigma_{-1}(v), \sigma_{-1}\left(v^{\prime}\right)\right], \sigma_{-1}\left(v^{\prime \prime}\right)\right]+A_{0},
\end{aligned}
$$

the Jacobi identity on $A$ yields the equation

$$
\left[\Lambda\left(v, v^{\prime}\right), v^{\prime \prime}\right]+\left[\Lambda\left(v^{\prime}, v^{\prime \prime}\right), v\right]+\left[\Lambda\left(v^{\prime \prime}, v\right), v^{\prime}\right]=0
$$

for all $v, v^{\prime}, v^{\prime \prime} \in G_{-1}$. So Proposition 4.6(2) applies.

Set $\pi_{2}:(\mathfrak{g l}(2) \otimes A(1 ; \underline{1})) \oplus(F \operatorname{Id} \otimes W(1 ; \underline{1})) \rightarrow W(1 ; \underline{1})$, the projection onto the second summand, and let $\Lambda_{2}:=\left(\pi_{2} \circ \Lambda\right)$.

4) If $\Lambda_{2}=0$ then $\left[V_{-1}, V_{-1}\right] \subset V_{0}^{\prime}$, a contradiction. So assume that $\Lambda_{2} \neq 0$.

We observe that the isomorphism

$$
W(1 ; \underline{1}) \cong(\mathfrak{s l}(2) \otimes A(1 ; \underline{1})) \oplus(F \operatorname{Id} \otimes A(1 ; \underline{1})) /(\mathfrak{s l}(2) \otimes A(1 ; \underline{1}))
$$

gives $W(1 ; \underline{1})$ a $G_{0}$-module structure. The multiplication on $A$ gives rise to a bilinear mapping

$$
\Phi:\left(\sum_{j \in \mathbb{F}_{p}} A_{ \pm \alpha / 2+j \alpha_{2}}\right) \times\left(\sum_{j \in \mathbb{F}_{p}} A_{ \pm \alpha / 2+j \alpha_{2}}\right) \rightarrow A_{0} .
$$


Since $\left(\sum_{j \in \mathbb{F}_{p}} A_{ \pm \alpha / 2+j \alpha_{2}}\right) \cap A_{0} \subset A_{1},\left[A_{1}, A\right] \subset V_{0}^{\prime}+A_{1}$, and $\left[\sum_{j \in \mathbb{F}_{p}} A_{ \pm \alpha / 2+j \alpha_{2}}, V_{0}\right]$ $\subset\left(\sum_{j \in \mathbb{F}_{p}} A_{ \pm \alpha / 2+j \alpha_{2}}\right)+A_{1}, \Phi$ induces a $G_{0}$-invariant mapping

$$
\tilde{\Phi}: G_{-1} \times G_{-1} \rightarrow G_{0} /(\mathfrak{s l}(2) \otimes A(1 ; \underline{1})) \cong W(1 ; \underline{1}) .
$$

Note that

$$
\operatorname{Id} \otimes \tilde{\Phi}\left(v, v^{\prime}\right)=\left[\sigma_{-1}(v), \sigma_{-1}\left(v^{\prime}\right)\right]+\left(V_{0}^{\prime}+A_{1}\right) .
$$

Hence $\tilde{\Phi}=\Lambda_{2}$, and therefore $\Lambda_{2}$ is $G_{0}$-invariant.

We now normalize $T$. By the initial assumption, $\mathfrak{s l}(2) \otimes A(1 ; \underline{1})$ is $T$-simple. According to [14, Theorem IV.3] $T$ is conjugate to

$$
(F h \otimes 1) \oplus(F \operatorname{Id} \otimes(1+x) d / d x) .
$$

Set $t_{0}=(1+x) d / d x$.

Next we are going to normalize $U$. Let $\tilde{u} \in U \otimes A(1 ; \underline{1})$ be any eigenvector with respect to $T$. Since $\left[t_{0},\left(\operatorname{Id} \otimes(1+x)^{i}\right)(\tilde{u})\right]=\left(\operatorname{Id} \otimes(1+x)^{i}\right)\left(\left[t_{0}, \tilde{u}\right]\right)+i\left(\operatorname{Id} \otimes(1+x)^{i}\right)(\tilde{u})$ (cf. Theorem 4.5(3)), there is $i \in \mathbb{F}_{p}$ so that $\left(\operatorname{Id} \otimes(1+x)^{i}\right)(\tilde{u})$ is annihilated by $t_{0}$. As $\operatorname{Id} \otimes(1+x)^{i}$ acts invertibly on $U \otimes A(1 ; \underline{1})$ we have that $U \otimes A(1 ; \underline{1}) \cong$ $C_{U \otimes A(1 ; 1)}\left(F t_{0}\right) \otimes A(1 ; \underline{1})$ as $(\mathfrak{s l}(2) \otimes A(1 ; \underline{1})+T)$-module. Thus we may assume that $\mathfrak{s l}(2) \otimes A(1 ; \underline{1})+T=(\mathfrak{s l}(2) \otimes A(1 ; \underline{1})) \oplus(F \mathrm{Id} \otimes(1+x) d / d x)$ acts on $U \otimes A(1 ; \underline{1})$ by the natural action.

Set $f:=1+x$ in Proposition $4.6(2)$ and recall that $\Lambda_{2}$ is $G_{0}$-invariant. As $W(1 ; \underline{1})$ is considered as a trivial $\mathfrak{s l}(2) \otimes A(1 ; \underline{1})$-module, we have

$$
\begin{aligned}
0 \neq & \Lambda_{2}(U \otimes A(1 ; \underline{1}), U \otimes A(1 ; \underline{1})) \\
= & \Lambda_{2}(U \otimes A(1 ; \underline{1}),[h \otimes A(1 ; \underline{1}), U \otimes 1]) \\
= & \Lambda_{2}([h \otimes A(1 ; \underline{1}), U \otimes A(1 ; \underline{1})], U \otimes 1) \\
& +\left[h \otimes A(1 ; \underline{1}), \Lambda_{2}(U \otimes A(1 ; \underline{1}), U \otimes 1)\right] \\
= & \Lambda_{2}(U \otimes A(1 ; \underline{1}), U \otimes 1),
\end{aligned}
$$

and

$$
\begin{aligned}
\Lambda_{2}\left(u \otimes(1+x)^{i}, u^{\prime} \otimes 1\right)= & (i-2)(1+x)^{i} \Lambda_{2}\left(u \otimes 1, u^{\prime} \otimes 1\right) \\
& +(3-2 i)(1+x)^{i-1} \Lambda_{2}\left(u \otimes(1+x), u^{\prime} \otimes 1\right) \\
& +(i-1)(1+x)^{i-2} \Lambda_{2}\left(u \otimes(1+x)^{2}, u^{\prime} \otimes 1\right) \\
& +(1+x) \Lambda_{2}\left(u \otimes(1+x)^{i-1}, u^{\prime} \otimes 1\right) .
\end{aligned}
$$

Inductively, we obtain

$$
\begin{aligned}
\Lambda_{2}(u \otimes & \left.(1+x)^{i}, u^{\prime} \otimes 1\right) \\
= & \frac{(i-1)(i-2)}{2}(1+x)^{i} \Lambda_{2}\left(u \otimes 1, u^{\prime} \otimes 1\right) \\
& +i(2-i)(1+x)^{i-1} \Lambda_{2}\left(u \otimes(1+x), u^{\prime} \otimes 1\right) \\
& +\frac{i(i-1)}{2}(1+x)^{i-2} \Lambda_{2}\left(u \otimes(1+x)^{2}, u^{\prime} \otimes 1\right) .
\end{aligned}
$$

Next choose $u, u^{\prime} \in U$ such that $[h, u]=u,\left[h, u^{\prime}\right]=-u^{\prime}$. Considering eigenvalues, one finds $s_{0}, s_{1}, s_{2} \in F$ such that

$$
\Lambda_{2}\left(u \otimes(1+x)^{k}, u^{\prime} \otimes 1\right)=s_{k}(1+x)^{k+1} d / d x, \quad k=0,1,2 .
$$


Then

$$
\begin{aligned}
\Lambda_{2}(u & \left.\otimes(1+x)^{i}, u^{\prime} \otimes 1\right) \\
& =\left(\frac{s_{0}(i-1)(i-2)}{2}+s_{1} i(2-i)+\frac{s_{2} i(i-1)}{2}\right)(1+x)^{i+1} d / d x .
\end{aligned}
$$

As $\Lambda_{2} \neq 0$ by assumption, at least one of $s_{0}, s_{1}, s_{2}$ has to be nonzero. Then the above coefficient, considered as a polynomial in $i$, is a nonzero polynomial of degree $\leq 2$. Consequently, it has at most 2 different zeros. We obtain

$$
\operatorname{dim} \Lambda_{2}\left(G_{-1}, G_{-1}\right) \geq p-2 .
$$

But $W(1 ; \underline{1})$ contains no proper $F(1+x) d / d x$-invariant subalgebra of this dimension.

As a result, $G_{0} /(\mathfrak{s l}(2) \otimes A(1 ; \underline{1})) \cong W(1 ; \underline{1})$. This contradicts the result mentioned in (1).

Lemma 4.8. Let $A$ and $G$ be as in Lemma 8.10.3. Then there is a distinguished maximal subalgebra $A_{0}^{\prime}(T)$ satisfying $\operatorname{dim} A_{0}(T)<\operatorname{dim} A_{0}^{\prime}(T)$.

Proof. 1) According to Lemmas 8.10.5 and 8.10.6 we have $G_{-2}=(0)$. According to Theorem 4.5 and Lemma 8.10.3, set $G_{-1}=U \otimes A(1 ; \underline{1})$, where $U \otimes 1$ is an irreducible 2-dimensional $(\mathfrak{s l}(2) \otimes 1)$-module.

2) Choose $T$-invariant vector spaces

$$
\tilde{V}_{-1}^{\prime} \subset \tilde{V}_{-1} \subset V_{-1} \subset \sum_{j \in \mathbb{F}_{p}} A_{ \pm \alpha / 2+j \alpha_{2}}, \quad V_{0} \subset \tilde{V}_{0} \subset \sum_{j \in \mathbb{F}_{p}} A_{ \pm \alpha+j \alpha_{2}}+\sum_{j \in \mathbb{F}_{p}} A_{j \alpha_{2}},
$$

such that

$$
\begin{aligned}
V_{-1}+A_{0} / A_{0} & =G_{-1}, \quad \tilde{V}_{-1}+A_{0} / A_{0}=U \otimes\left(F x^{p-2}+F x^{p-1}\right), \\
\tilde{V}_{-1}^{\prime}+A_{0} / A_{0} & =U \otimes F x^{p-1}, \quad V_{0}+A_{1} / A_{1}=\mathfrak{s l}(2) \otimes A(1 ; \underline{1}), \\
\tilde{V}_{0}+A_{1} / A_{1} & =(\mathfrak{s l}(2) \otimes A(1 ; \underline{1})) \oplus\left(F \operatorname{Id} \otimes W(1 ; \underline{1})_{(0)}\right) .
\end{aligned}
$$

Considering the associated graded Lie algebra $G$ and the root space decomposition of $A$, one concludes that

$$
V_{-1} \cap A_{0} \subset A_{1}, \quad\left[V_{-1}, V_{-1}\right] \subset A_{0}, \quad\left[\tilde{V}_{-1}^{\prime}, \tilde{V}_{0}\right] \subset \tilde{V}_{-1}^{\prime}+A_{1}, \quad\left[V_{0}, A_{0}\right] \subset V_{0}+A_{1} .
$$

Every element $\varphi \in G_{1}$ satisfies $\left[[\varphi, v], v^{\prime}\right]=\left[\left[\varphi, v^{\prime}\right], v\right]$ for all $v, v^{\prime} \in G_{-1}$. Thus $\varphi$ gives rise to a Cartan prolongation (again denoted by $\varphi$ ), and Proposition 4.6(1) shows that

$$
\left[G_{1}, U \otimes A(1 ; \underline{1})_{(1)}\right] \subset(\mathfrak{s l}(2) \otimes A(1 ; \underline{1})) \oplus\left(F \operatorname{Id} \otimes W(1 ; \underline{1})_{(0)}\right) .
$$

Consequently,

$$
\left[A_{1}, \tilde{V}_{-1}\right] \subset \tilde{V}_{0}+A_{1}
$$

Let $v \in V_{0}$ be an inverse image of $h \otimes x \in \mathfrak{s l}(2) \otimes A(1 ; \underline{1})$. Since $h \otimes 1$ acts invertibly on $U \otimes 1$ one has $\tilde{V}_{-1}^{\prime} \subset\left[v, \tilde{V}_{-1}\right]+A_{1}$. Considering the associated graded Lie algebra $G$ and the root space decomposition of $A$, one also concludes that $\left[v, \tilde{V}_{-1}^{\prime}\right] \subset A_{1}$. Then

$$
\begin{aligned}
{\left[\tilde{V}_{-1}^{\prime}, \tilde{V}_{-1}^{\prime}\right] } & \subset\left[v,\left[\tilde{V}_{-1}^{\prime}, \tilde{V}_{-1}+A_{1}\right]\right]+\left[\left[v, \tilde{V}_{-1}^{\prime}\right], \tilde{V}_{-1}+A_{1}\right] \\
& \subset\left[v, A_{0}\right]+\left[A_{1}, \tilde{V}_{-1}+A_{1}\right] \subset \tilde{V}_{0}+A_{1} .
\end{aligned}
$$


Therefore $A_{0}^{\prime}(T):=\tilde{V}_{-1}^{\prime}+\tilde{V}_{0}+A_{1}$ is a subalgebra of $A$. As

$$
\operatorname{dim} G_{0} /(\mathfrak{s l}(2) \otimes A(1 ; \underline{1})) \oplus\left(F \operatorname{Id} \otimes W(1 ; \underline{1})_{(0)}\right)=1
$$

and $\operatorname{dim} U=2$, we have $\operatorname{dim} A_{0}^{\prime}(T)=1+\operatorname{dim} A_{0}(T)$.

3) The assumption on $T$ yields that $\tilde{H} \subset A_{0}^{\prime}(T)$. Clearly, $R_{\mu} \subset\left(V_{-1}+V_{0}+A_{1}\right) \cap$ $A_{0} \subset A_{0}^{\prime}$ whenever $\mu \notin \mathbb{F}_{p} \alpha_{2}$. By definition, $\left[R_{i \alpha_{2}}+A_{1} / A_{1}, F h \otimes A(1 ; \underline{1})\right] \cap \tilde{H}$ acts nilpotently on $G$. Then $R_{i \alpha_{2}}+A_{1} / A_{1} \subset(\mathfrak{s l}(2) \otimes A(1 ; \underline{1})) \oplus\left(F \operatorname{Id} \otimes W(1 ; \underline{1})_{(0)}\right)$, so $R_{i \alpha_{2}} \subset A_{0}^{\prime}$. This proves the lemma.

The following corollary is immediate.

Corollary 4.9. Suppose $A_{0}(T)$ is a distinguished subalgebra of maximal dimension.

1) $G_{0}$ cannot be one the algebras listed in Theorem 4.1.1(b) of [3].

2) $G_{0}$ cannot be as in case 3) of Proposition 4.3.

The proofs of Lemmas 8.11.1 and 8.11.2 are valid in the present case without changes. This observation proves

Lemma 4.10. Case 2) of Proposition 4.3 is impossible.

The final result of this and the previous section is the following.

Theorem 4.11. Let $L$ be a simple Lie algebra satisfying $(A ; 3)$, and let $L_{p}$ be a semisimple p-envelope of $L$. Suppose that every maximal 2-dimensional torus $U \subset L_{p}$ has the property that $C_{L}(U)$ is a CSA of $L$ and $U$ is contained in the p-envelope $\widetilde{C_{L}(U)}$ of $C_{L}(U)$ in $L_{p}$.

Then $L$ is of Cartan type $S(3 ; \underline{n} ; \Psi)^{(1)}$ or $H(4 ; \underline{n} ; \Psi)^{(2)}$.

Proof. Let $T$ denote a maximal 2-dimensional torus having maximal number of proper roots, and let $H:=C_{L}(T)$. Put $A:=L+\tilde{H}$ and let $A_{0}$ denote a subalgebra of $A T$ of maximal dimension which contains $R(A)$. Theorem 3.8 shows that $\operatorname{rad} A_{0}$ acts nilpotently. Thus we are in the case of this section 4 . As a result of the preceding deliberations $G_{1} \neq(0)$ and $\tilde{G}_{0}=G_{0}$ is classical simple, or else

$$
\tilde{G}_{0}=G_{0} \cong \mathfrak{s l}(2) \oplus \mathfrak{s l}(2), A=A_{-1}, N(G)=(0), G_{0} \text { acts faithfully on } G_{1} .
$$

In the latter case the recognition theorem for graded algebras implies that $G$ is either classical or of Cartan type. But there is no $G$ of classical or Cartan type satisfying $G_{0} \cong \mathfrak{s l}(2) \oplus \mathfrak{s l}(2)$ (cf. [3, p.228]).

Thus $G_{0}$ is classical simple and $G_{1} \neq(0)$. The arguments presented in [3, p.228] yield that for some choice of $\underline{n}, S(3 ; \underline{n})^{(1)} \subset G^{\prime} / N(G) \subset S(3 ; \underline{n})$ or $H(4 ; \underline{n})^{(2)} \subset$ $G^{\prime} / N(G) \subset H(4 ; \underline{n})$. By definition $G^{\prime}$ is generated by $\sum_{i \leq 1} G_{i}$, which means that $\underline{n}=\underline{1}$. Then [3, p.229] shows that $N(G)=(0)$ and $S(3 ; \underline{1})^{(1)} \subset G^{\prime} \subset S(3 ; \underline{1})$ or $H(4 ; \underline{1})^{(2)} \subset G^{\prime} \subset H(4 ; \underline{1})$. If the grading of $G^{\prime}$ is the reverse to the canonical grading, then $\operatorname{dim} \sum_{i<0} G_{i}>7\left(p^{2}-p+6\right)$, which is impossible. Thus the grading is the canonical one. Then $G_{-2}=G_{-2}^{\prime}=(0)$. Thus the recognition theorem for filtered Lie algebras applies and gives the result. 


\section{The Classification, PART I}

The results of Theorems 2.4 and 4.11 yield that if $L$ is simple and satisfies $(A ; 3)$ then $L$ is one of the following:

(H2) $H(2 ; \underline{n} ; \Psi)^{(2)}$,

(S3) $S(3 ; \underline{n} ; \Psi)^{(1)}$,

(H4) $H(4 ; \underline{n} ; \Psi)^{(2)}$.

We are now going to figure out which of these algebras do have the property $(A ; 3)$.

Lemma 5.1. Let $L_{p}$ be the $p$-envelope of $L$ in $\operatorname{Der} L$, and let $T \subset L_{p}$ be a torus of dimension 3. Then $T \cap L=(0)$. Consequently,

$$
\operatorname{dim}(\operatorname{Der} L) / L \geq \operatorname{dim} L_{p} / L \geq 3=T R(L) .
$$

Proof. Theorem 1.5 shows that $C_{L}(T)$ acts nilpotently on $L$. Thus $T \cap L$ acts trivially on $L$, whence $T \cap L \subset C(L)=(0)$.

(H2): It has been mentioned in $[3, \S 2]$ that there are 3 types of Hamiltonian algebras in 2 "generators", namely $H(2 ; \underline{n})^{(2)}, H(2 ; \underline{n} ; \Phi(\tau))^{(1)}$ and $H(2 ; \underline{n} ; \Delta)$.

(a) $H(2 ; \underline{n})^{(2)}$ : According to $[3,(2.2 .3)]$ one has

$$
\operatorname{TR}\left(H(2 ; \underline{n})^{(2)}\right) \geq n_{1}+n_{2}-1 \text {. }
$$

Note that (cf. $[3,(2.1 .8)])$

$$
H(2 ; \underline{n})_{p}^{(2)}=H(2 ; \underline{n})^{(2)}+\sum_{i=1}^{n_{1}-1} F D_{1}^{p^{i}}+\sum_{i=1}^{n_{2}-1} F D_{2}^{p^{i}} .
$$

According to Lemma 5.1 these algebras do not occur.

(b) $H(2 ; \underline{n} ; \Phi(\tau))^{(1)}$ : According to $[3,(2.2 .3)]$ the algebra $H(2 ; \underline{n} ; \Phi(\tau))^{(1)}$ has toral rank

$$
\operatorname{TR}\left(H(2 ; \underline{n} ; \Phi(\tau))^{(1)}\right) \geq n_{1}+n_{2} .
$$

Thus in the present case $n_{1}+n_{2} \leq 3$. As $T R\left(H(2 ; \underline{1} ; \Phi(\tau))^{(1)}\right)=2$ and as we may assume by symmetry $n_{1} \geq n_{2}$, the only candidate to satisfy $(A ; 3)$ is $H(2 ;(2,1) ; \Phi(\tau))^{(1)}$. We will see below that this algebra in fact has this property.

(c) $H(2 ; \underline{n} ; \Delta)$ : According to $[3,(2.2 .3),(2.1 .7)]$ we have

$$
\begin{aligned}
\operatorname{TR}(H(2 ; \underline{n} ; \Delta)) & \geq n_{1}+n_{2}, \\
\operatorname{dim}(\operatorname{Der} H(2 ; \underline{n} ; \Delta) / H(2 ; \underline{n} ; \Delta)) & =\left(n_{1}-1\right)+\left(n_{2}-1\right)+1 .
\end{aligned}
$$

Thus these algebras do not satisfy $(A ; 3)$.

(S3): The isomorphism types of the special algebras have been determined in [26]. There are 3 types of special algebras in 3 "generators", namely $S(3 ; \underline{n})^{(1)}$, $S(3 ; \underline{n} ; \Phi(i))^{(1)}$ and $S(3 ; \underline{n} ; \Phi(\tau))^{(1)}$.

(a) $S(3 ; \underline{n})^{(1)}:$ Let $i, j, k$ be pairwise different indices. Set

$$
W_{i}:=\sum_{r=0}^{p^{n_{i}}-1} F\left(x_{i}^{(r)} D_{i}-x_{i}^{(r-1)} x_{k} D_{k}\right) \subset S(3 ; \underline{n})^{(1)} .
$$

Then $W_{i} \cong W\left(1 ; \underline{n}_{i}\right)$ and

$$
\left[W_{i}, W_{j}\right]=(0), \quad W_{i} \cap W_{j}=(0) .
$$


It is shown in $[14$, section $\mathrm{V}]$ that there are tori $T_{i}, T_{j}$ in $\left(W_{i}\right)_{p}$ and $\left(W_{j}\right)_{p}$ of dimension $n_{i}$ and $n_{j}$, respectively, and that $T_{i} \cap W_{i} \neq(0)$. If $S(3 ; \underline{n})^{(1)}$ were to satisfy $(A ; 3)$ then, due to Lemma $5.1, \operatorname{dim}\left(T_{i} \oplus T_{j}\right)=n_{i}+n_{j} \leq 2$. This shows $\underline{n}=\underline{1}$. But $S(3 ; \underline{1})^{(1)}$ has toral rank 2 . As a consequence, no algebra of this type satisfies $(A ; 3)$.

(b) $S(3 ; \underline{n} ; \Phi(i))^{(1)}$ : This algebra is defined (cf. [26]) by

$$
S(3 ; \underline{n} ; \Phi(i)):=\left\{E \in W(3 ; \underline{n}) \mid E\left(\sum_{r=0}^{\infty}\left(x_{i}^{\left(p^{n_{i}}\right)}\right)^{(r)} d x_{1} \wedge d x_{2} \wedge d x_{3}\right)=0\right\} .
$$

By symmetry we may set $i=1$. Then $S(3 ; \underline{n} ; \Phi(1))$ contains the elements

$$
D_{2}, \quad x_{1} x_{2} D_{1}-x_{2}^{(2)} D_{2}, \quad x_{2}^{(2)} D_{2}-x_{2} x_{3} D_{3}, \quad x_{2} D_{1}-x_{1}^{\left(p^{n_{1}}-1\right)} x_{2}^{(2)} D_{2} .
$$

Define elements $h_{1}, h_{2}, \tilde{D} \in S(3 ; \underline{n} ; \Phi(1))^{(1)}$ by

$$
\begin{aligned}
h_{1} & :=\left[D_{2}, x_{1} x_{2} D_{1}-x_{2}^{(2)} D_{2}\right]=x_{1} D_{1}-x_{2} D_{2}, \\
h_{2} & :=\left[D_{2}, x_{2}^{(2)} D_{2}-x_{2} x_{3} D_{3}\right]=x_{2} D_{2}-x_{3} D_{3}, \\
\tilde{D} & :=\left[D_{2}, x_{2} D_{1}-x_{1}^{\left(p^{n_{1}}-1\right)} x_{2}^{(2)} D_{2}\right]=D_{1}-x_{1}^{\left(p^{n_{1}}-1\right)} x_{2} D_{2} .
\end{aligned}
$$

Note that $h_{1}, h_{2}$ are toral elements, and

$$
\left[h_{1}, h_{2}\right]=0, \quad\left[\tilde{D}^{p}, h_{i}\right] \in F(\operatorname{ad} \tilde{D})^{p-1}\left(\left[\tilde{D}, h_{i}\right]\right)=0 .
$$

It is easy to check that

$$
\tilde{D}^{p^{n_{1}}}\left(x_{2}\right)=-x_{2}, \quad \tilde{D}^{p^{n_{1}}}\left(x_{1}\right)=\tilde{D}^{p^{n_{1}}}\left(x_{3}\right)=0 .
$$

Thus the $p$-subalgebra generated by $\tilde{D}^{p}$ contains a toral element $t$ satisfying $t\left(x_{1}\right)=t\left(x_{3}\right)=0$. Then

$$
T:=F\left(x_{1} D_{1}-x_{2} D_{2}\right) \oplus F\left(x_{2} D_{2}-x_{3} D_{3}\right) \oplus F t
$$

is a 3 -dimensional torus for which $T \cap S(3 ; \underline{n} ; \Phi(i))^{(1)} \neq(0)$. Therefore these algebras do not satisfy $(A ; 3)$.

(c) $S(3 ; \underline{n} ; \Phi(\tau))^{(1)}$ : This algebra is defined (cf. [26]) by

$$
\begin{aligned}
& S(3 ; \underline{n} ; \Phi(\tau)) \\
& \quad:=\left\{E \in W(3 ; \underline{n}) \mid E\left(\left(1+x_{1}^{\left(p^{n_{1}}-1\right)} x_{2}^{\left(p^{n_{2}}-1\right)} x_{3}^{\left(p^{n_{3}}-1\right)}\right) d x_{1} \wedge d x_{2} \wedge d x_{3}\right)=0\right\} .
\end{aligned}
$$

Then $S(3 ; \underline{n} ; \Phi(\tau))$ contains the elements

$$
\begin{aligned}
& \left(1-x_{1}^{\left(p^{n_{1}}-1\right)} x_{2}^{\left(p^{n_{2}}-1\right)} x_{3}^{\left(p^{n_{3}}-1\right)}\right) D_{i}, \quad i=1,2,3, \\
& x_{i}^{(r)} D_{i}-x_{i}^{(r-1)} x_{j} D_{j}, \quad i \neq j, \quad 0<r<p^{n_{i}} .
\end{aligned}
$$

If $\underline{n}>\underline{1}$ then by symmetry we may assume $n_{1}>1$. The algebra

$$
\begin{array}{rl}
W_{1}:=\sum_{r=1}^{p^{n_{1}}-1} & F\left(x_{1}^{(r)} D_{1}-x_{1}^{(r-1)} x_{2} D_{2}\right) \\
& +F\left(1-x_{1}^{\left(p^{n_{1}}-1\right)} x_{2}^{\left(p^{n_{2}}-1\right)} x_{3}^{\left(p^{n_{3}}-1\right)}\right) D_{1} \cong W\left(1 ; \underline{n_{1}}\right)
\end{array}
$$

has toral rank $n_{1}$. Since $x_{2} D_{2}-x_{3} D_{3} \in S(3 ; \underline{n} ; \Phi(\tau))^{(1)}$ and

$$
\left[W_{1}, x_{2} D_{2}-x_{3} D_{3}\right]=(0)
$$


the $p$-envelope of $S(3 ; \underline{n} ; \Phi(\tau))^{(1)}$ contains a torus $T$ of dimension $n_{1}+1 \geq 3$, which intersects $S(3 ; \underline{n} ; \Phi(\tau))^{(1)}$ nontrivially. Lemma 5.1 shows that this is impossible. Consequently, $n_{i}=1$ for all $i$. We will see below that $S(3 ; \underline{1} ; \Phi(\tau))^{(1)}$ in fact satisfies $(A ; 3)$.

(H 4): Let $L_{p}$ be the $p$-envelope of $L$ in Der $L$, and let $T \subset L_{p}$ be a torus of dimension 3. Let $\left(L_{(i)}\right)_{i \geq-1}$ denote the canonical filtration of $L$ as a Cartan type Lie algebra. Set

$$
\mathfrak{N}:=\left\{x \in L_{p} \mid\left[x, L_{(0)}\right] \subset L_{(0)}\right\}=\operatorname{Nor}_{L_{p}} L_{(0)} .
$$

Lemma 5.2. If $t \in \mathfrak{N}$ satisfies $\left[t, L_{(0)}\right] \subset L_{(1)}$ then $t$ is ad-nilpotent.

Proof. Suppose that $t$ is not ad-nilpotent. By decomposing $t$ into the $[p]$-nilpotent and $[p]$-semisimple part we may assume that $t$ is semisimple.

Consider the associated graded algebra gr $L=\sum_{i>-1} \operatorname{gr}_{i} L$. Since $t \in \mathfrak{N}$, it stabilizes the filtration and hence acts on gr $L$. By assumption, $\left[t, \operatorname{gr}_{0} L\right]=(0)$. As $\mathrm{gr}_{-1} L$ is an irreducible $\mathrm{gr}_{0} L$-module, this implies

$$
\operatorname{ad}_{\mathrm{gr}_{-1} L} t=\alpha \operatorname{Idgr}_{-1} L \quad \text { for some } \alpha \in F \text {. }
$$

Suppose $\alpha \neq 0$. Then

$$
C_{L}(t) \subset L_{(0)} \subset C_{L}(t)+L_{(1)}
$$

Consequently,

$$
C_{L}(t) / \operatorname{rad} C_{L}(t) \cong \operatorname{gr}_{0} L \cong \mathfrak{s p}(4) .
$$

Since $\mathfrak{s p}(4)$ has toral rank $2, \mathfrak{N} \subset L_{p}$ has a 3 -dimensional torus containing $t$, and $C_{L}(t)$ is a 2 -section with respect to this torus. According to Theorem 1.5 no 2section has classical semisimple quotient.

Thus $\alpha=0$. Then $[t, L] \subset L_{(0)}$. The semisimplicity of ad $t$ implies that $L$ decomposes as

$$
L=[t, L]+C_{L}(t) .
$$

Therefore $[t, L]+[[t, L],[t, L]]$ is an ideal of $L$, which according to the above result is contained in $L_{(0)}$. Hence $[t, L]=(0)$, whence $t=0$.

We consider the associated graded algebra gr $L$, which satisfies the compatibility condition

$$
H(4 ; \underline{n})^{(2)} \subset \operatorname{gr} L \subset H(4 ; \underline{n}) .
$$

Thus $\operatorname{gr}_{0} L \cong \mathfrak{s p}(4)$ has a 2 -dimensional torus

$$
R=F\left(x_{1} D_{1}-x_{3} D_{3}\right) \oplus F\left(x_{2} D_{2}-x_{4} D_{4}\right) .
$$

With respect to this torus gr $L$ has 1-sections satisfying

$$
\left((\operatorname{gr} L)\left(\alpha_{i}\right) / \operatorname{rad}(\operatorname{gr} L)\left(\alpha_{i}\right)\right)^{(2)} \cong H\left(2 ;\left(n_{1+i}, n_{3+i}\right)\right)^{(2)}, \quad i=1,2 .
$$

Lift this information to $L$. The $p$-envelope $M$ of $L_{(0)}$ in $L_{p} \subset \operatorname{Der} H(4 ; \underline{n} ; \Psi)^{(2)}$ contains a 2-dimensional torus

$$
\widetilde{R}=F\left(x_{1} D_{1}-x_{3} D_{3}+E_{1}\right) \oplus F\left(x_{2} D_{2}-x_{4} D_{4}+E_{2}\right)
$$


with $E_{1}, E_{2} \in W(4 ; \underline{n})_{(1)}$. The corresponding 1-sections of $L$ have the property $\operatorname{rad} L\left(\alpha_{i}\right) \subset W(4 ; \underline{n})_{(0)}$, and the associated graded algebras $\operatorname{gr}\left(L\left(\alpha_{i}\right)\right)$ of these 1sections $L\left(\alpha_{i}\right)$ are of the same types as $(\operatorname{gr} L)\left(\alpha_{i}\right)$. Then $L$ itself has 1 -sections with respect to $\widetilde{R}$ for which

$$
\left(L\left(\alpha_{i}\right) / \operatorname{rad} L\left(\alpha_{i}\right)\right)^{(2)} \cong H\left(2 ;\left(n_{1+i}, n_{3+i}\right) ; \Psi_{i}\right)^{(2)}, \quad i=1,2,
$$

with suitable automorphisms $\Psi_{1}, \Psi_{2}$. If $\widetilde{R}$ is a maximal torus then Theorem 2.2 applies and yields $n_{1}=\ldots=n_{4}=1$. If $\widetilde{R}$ is not a maximal torus then Theorem 1.5 yields the same result. Thus we have $L=\mathfrak{H}^{(2)}$, where

$$
\mathfrak{H}:=\{D \in W(4 ; \underline{1}) \mid D(\omega)=0\}
$$

for some differential form

$$
\omega=\sum_{1 \leq i, j \leq 4} \omega_{i j} d x_{i} \wedge d x_{j}
$$

According to $[7]$ the following are true:

$$
\omega_{i j}=-\omega_{j i}, \quad D_{i}\left(\omega_{j k}\right)+D_{j}\left(\omega_{k i}\right)+D_{k}\left(\omega_{i j}\right)=0, \quad \operatorname{det}\left(\omega_{i j}\right) \text { is invertible. }
$$

Set

$$
\left(\varphi_{i j}\right):=\left(\omega_{i j}\right)^{-1}
$$

and

$$
\mathfrak{D}(f):=\sum_{i, j} \varphi_{i j} D_{j}(f) D_{i}
$$

Then (cf. [7])

$$
\mathfrak{D}(f) \in \mathfrak{H} \quad \text { for all } \quad f \in A(4 ; \underline{1})
$$

and

$$
[\mathfrak{D}(f), \mathfrak{D}(g)]=\mathfrak{D}\left(\sum_{r, s} \varphi_{s r} D_{r}(f) D_{s}(g)\right) .
$$

(a) If not all of the coefficients of $\omega$ are in $A(4 ; \underline{1})$, we may assume by [4, Theorem 1] (where we may set $s=1$ by symmetry) that

$$
\begin{aligned}
\left(\omega_{i j}\right) \equiv\left(1+x_{1}^{(p)}\right) & \left(\begin{array}{rrrr}
0 & 0 & 1 & 0 \\
0 & 0 & 0 & 1 \\
-1 & 0 & 0 & 0 \\
0 & -1 & 0 & 0
\end{array}\right) \\
& +\frac{1}{2}\left(\begin{array}{cccc}
0 & -x_{1}^{(p-1)} x_{4} & 0 & x_{1}^{(p-1)} x_{2} \\
x_{1}^{(p-1)} x_{4} & 0 & 0 & 0 \\
0 & 0 & 0 & 0 \\
-x_{1}^{(p-1)} x_{2} & 0 & 0 & 0
\end{array}\right)
\end{aligned}
$$

modulo terms of degree $\geq 2 p-2$. It follows that

$$
\left(\varphi_{i j}\right) \equiv\left(\begin{array}{cccc}
0 & 0 & -1+x_{1}^{(p)} & 0 \\
0 & 0 & \frac{1}{2} x_{1}^{(p-1)} x_{2} & -1+x_{1}^{(p)} \\
1-x_{1}^{(p)} & -\frac{1}{2} x_{1}^{(p-1)} x_{2} & 0 & -\frac{1}{2} x_{1}^{(p-1)} x_{4} \\
0 & 1-x_{1}^{(p)} & \frac{1}{2} x_{1}^{(p-1)} x_{4} & 0
\end{array}\right)
$$

modulo terms of degree $\geq 2 p-2$. 
Then

$$
\begin{aligned}
\mathfrak{D}\left(x_{1}\right)= & \left(1-x_{1}^{(p)}\right) D_{3}+\text { terms of degree } \geq 2 p-3, \\
\mathfrak{D}\left(x_{3}^{(2)}\right)= & x_{3}\left(\left(-1+x_{1}^{(p)}\right) D_{1}+\frac{1}{2} x_{1}^{(p-1)} x_{2} D_{2}+\frac{1}{2} x_{1}^{(p-1)} x_{4} D_{4}\right) \\
& \quad+\text { terms of degree } \geq 2 p-2, \\
\mathfrak{D}\left(x_{1} x_{3}\right)= & \left(1-x_{1}^{(p)}\right) x_{3} D_{3}+\left(-1+x_{1}^{(p)}\right) x_{1} D_{1} \\
& \quad+\text { terms of degree } \geq 2 p-2 .
\end{aligned}
$$

Now

$$
\begin{aligned}
{\left[\mathfrak{D}\left(x_{1} x_{3}\right), \mathfrak{D}\left(x_{1}\right)\right] } & \equiv\left(1-x_{1}^{(p)}\right)^{2}\left[x_{3} D_{3}-x_{1} D_{1}, D_{3}\right] \\
& \equiv-\left(1-2 x_{1}^{(p)}\right) D_{3}
\end{aligned}
$$

modulo terms of degree $\geq 2 p-3$,

$$
\begin{aligned}
{\left[\mathfrak{D}\left(x_{1} x_{3}\right), \mathfrak{D}\left(x_{3}^{(2)}\right)\right] \equiv } & -2\left(1-2 x_{1}^{(p)}\right) x_{3} D_{1}-2 x_{1}^{(p-1)} x_{3}^{(2)} D_{3} \\
& +x_{1}^{(p-1)} x_{2} x_{3} D_{2}+x_{1}^{(p-1)} x_{3} x_{4} D_{4}
\end{aligned}
$$

modulo terms of degree $\geq 2 p-2$, and

$$
\begin{aligned}
E= & {\left[\left[\mathfrak{D}\left(x_{1} x_{3}\right), \mathfrak{D}\left(x_{1}\right)\right],\left[\mathfrak{D}\left(x_{1} x_{3}\right), \mathfrak{D}\left(x_{3}^{(2)}\right)\right]\right] } \\
\equiv & {\left[\left(1-2 x_{1}^{(p)}\right) D_{3}, 2\left(1-2 x_{1}^{(p)}\right) x_{3} D_{1}\right] } \\
& -\left[D_{3},-2 x_{1}^{(p-1)} x_{3}^{(2)} D_{3}+x_{1}^{(p-1)} x_{2} x_{3} D_{2}+x_{1}^{(p-1)} x_{3} x_{4} D_{4}\right] \\
\equiv & 2\left(1-4 x_{1}^{(p)}\right) D_{1}+6 x_{1}^{(p-1)} x_{3} D_{3}-x_{1}^{(p-1)} x_{2} D_{2} \\
& \quad-x_{1}^{(p-1)} x_{4} D_{4}
\end{aligned}
$$

modulo terms of degree $\geq 2 p-3$.

Note that $E \in \mathfrak{H}^{(2)}=L \subset W(4 ; \underline{1})$, and the latter is a restricted Lie algebra. Since

$$
E^{p}\left(x_{1}\right) \equiv-8 x_{1}, E^{p}\left(x_{2}\right) \equiv-x_{2}, E^{p}\left(x_{3}\right) \equiv 6 x_{3}, E^{p}\left(x_{4}\right) \equiv-x_{4}
$$

modulo terms of degree $>1$, we obtain

$$
E^{p}=-8 x_{1} D_{1}-x_{2} D_{2}+6 x_{3} D_{3}-x_{4} D_{4}+E_{1},
$$

where $E_{1}$ has degree $>0$.

A similar computation shows that $L$ contains

$$
\begin{aligned}
{\left[\left[\mathfrak{D}\left(x_{1}\right), \mathfrak{D}\left(x_{1} x_{3}\right)\right],\left[\mathfrak{D}\left(x_{1} x_{3}\right), \mathfrak{D}\left(x_{1} x_{3}^{(2)}\right)\right]\right] } & \equiv\left[D_{3}, x_{3}^{(2)} D_{3}-x_{1} x_{3} D_{1}\right] \\
& \equiv x_{3} D_{3}-x_{1} D_{1}
\end{aligned}
$$

modulo terms of degree $>0$. Thus $x_{3} D_{3}-x_{1} D_{1}+E_{2} \in L$, where $\operatorname{deg} E_{2}>0$.

Therefore $\mathfrak{N}$ contains

$$
t=E^{p}-7\left(x_{3} D_{3}-x_{1} D_{1}+E_{2}\right)=-\sum_{i=1}^{4} x_{i} D_{i}+E_{1}-7 E_{2} .
$$

Then $t$ is a nonnilpotent element which maps $L_{(0)}$ into $L_{(1)}$. Lemma 5.2 shows that this case is impossible. 
(b) Thus we may assume that the coefficients of $\omega$ are contained in $A(4 ; \underline{1})$. We also may assume that $L_{p} \subset \operatorname{Der} L \subset W(4 ; \underline{1})$ is the restricted subalgebra generated by $L$. In particular,

$$
D(\omega)=0 \quad \forall D \in L_{p} .
$$

Let $T \subset L_{p}$ denote a 3 -dimensional torus and

$$
T_{\mathfrak{N}}:=T \cap \mathfrak{N} .
$$

Since $T R\left(\operatorname{gr}_{0} L\right)=T R(\mathfrak{s p}(4))=2$, the assumption " $T \subset \mathfrak{N}$ " would imply that $T$ contains an element $t \neq 0$ satisfying $\left[t, L_{(0)}\right] \subset L_{(1)}$. As this contradicts Lemma 5.2, we have $\operatorname{dim} T_{\mathfrak{N}} \leq 2$.

Suppose that $\operatorname{dim} T_{\mathfrak{N}}=2$. Again Lemma 5.2 shows that $T_{\mathfrak{N}}$ is a CSA of $\operatorname{gr}_{0} L$. However, $W(4 ; \underline{1}) / W(4 ; \underline{1})_{(0)}$ considered as a $\operatorname{gr}_{0} L$-module has no zero weight with respect to any of its maximal tori. This implies that $T \subset$ $W(4 ; \underline{1})_{(0)}$, whence $T=T_{\mathfrak{N}}$, a contradiction. Consequently,

$$
\operatorname{dim} T_{\mathfrak{N}} \leq 1
$$

Choose toral elements $t_{1}, t_{2}, t_{3} \in T$, linearly independent over $F$, such that $t_{1}, t_{2}$ are linearly independent mod $T_{\mathfrak{N}}$. Since $T$ acts on the truncated polynomial ring $F\left[x_{1}, \ldots, x_{4}\right]$ as derivations, there are generators $y_{1}, \ldots, y_{4}$ such that

$$
t_{i}\left(y_{j}\right)=\alpha_{i j}\left(y_{j}+\delta_{j}\right)
$$

where $\alpha_{i j} \in G F(p), \delta_{j} \in\{0,1\}$. By reindexing the $y_{j}$ we obtain $\alpha_{11} \neq 0$ and $\delta_{1}=1$. Adjusting $t_{1}$, we may assume $\alpha_{11}=1$. Set

$$
y_{j}^{\prime}:=\left(y_{1}+1\right)^{-\alpha_{1 j}}\left(y_{j}+\delta_{j}\right)-\delta_{j}, \quad j=2,3,4 .
$$

Then $t_{1}\left(y_{j}^{\prime}\right)=0$ for $j \neq 1$.

Next set $t_{2}^{\prime}=t_{2}-\alpha_{21} t_{1}$ to obtain $t_{2}^{\prime}\left(y_{1}\right)=0$, and then proceed similarly with $t_{2}^{\prime}$. Thus we may assume that

$$
t_{1}=\left(y_{1}+1\right) \partial_{1}, \quad t_{2}=\left(y_{2}+1\right) \partial_{2}, \quad t_{3}=\gamma\left(y_{3}+\delta_{3}\right) \partial_{3}+\delta\left(y_{4}+\delta_{4}\right) \partial_{4} .
$$

Set

$$
z_{i}:=\left(y_{i}+1\right), \quad i=1,2 .
$$

In terms of these generators $\omega$ has the following form (as it is annihilated by $T):$

$$
\begin{aligned}
\omega= & z_{1}^{p-1} z_{2}^{p-1} \tilde{\omega}_{12} d y_{1} \wedge d y_{2}+\sum_{i=3}^{4} z_{1}^{p-1} \tilde{\omega}_{1 i} d y_{1} \wedge d y_{i} \\
& +\sum_{i=3}^{4} z_{2}^{p-1} \tilde{\omega}_{2 i} d y_{2} \wedge d y_{i}+\tilde{\omega}_{34} d y_{3} \wedge d y_{4}
\end{aligned}
$$

where $\tilde{\omega}_{i j}$ depends only on $y_{3}, y_{4}$. Then

$$
\left(\omega_{i j}\right)=\left(\begin{array}{cccc}
0 & z_{1}^{p-1} z_{2}^{p-1} \tilde{\omega}_{12} & z_{1}^{p-1} \tilde{\omega}_{13} & z_{1}^{p-1} \tilde{\omega}_{14} \\
-z_{1}^{p-1} z_{2}^{p-1} \tilde{\omega}_{12} & 0 & z_{2}^{p-1} \tilde{\omega}_{23} & z_{2}^{p-1} \tilde{\omega}_{24} \\
-z_{1}^{p-1} \tilde{\omega}_{13} & -z_{2}^{p-1} \tilde{\omega}_{23} & 0 & \tilde{\omega}_{34} \\
-z_{1}^{p-1} \tilde{\omega}_{14} & -z_{2}^{p-1} \tilde{\omega}_{24} & -\tilde{\omega}_{34} & 0
\end{array}\right)
$$

and

$$
\operatorname{det}\left(\omega_{i j}\right)=\left(z_{1}^{p-1} z_{2}^{p-1}\right)^{2} f\left(y_{3}, y_{4}\right)
$$


with some invertible polynomial depending on $y_{3}, y_{4}$ only. In order to compute $\left(\omega_{i j}\right)^{-1}=\left(\varphi_{i j}\right)$ we apply the well known formula for inverses: Let $A_{i j}$ denote the $3 \times 3$ submatrix of $\left(\omega_{k \ell}\right)$ where the $i$-th row and $j$-th column are deleted, set $b_{i j}:=(-1)^{i+j} \operatorname{det} A_{j i}$, and observe that

$$
\varphi_{i j}=\left(\operatorname{det}\left(\omega_{k \ell}\right)\right)^{-1} b_{i j} .
$$

A straightforward calculation show that

$$
\left(\varphi_{i j}\right)=\left(\begin{array}{cccc}
0 & z_{1} z_{2} \varphi_{12}^{\prime} & z_{1} \varphi_{13}^{\prime} & z_{1} \varphi_{14}^{\prime} \\
-z_{1} z_{2} \varphi_{12}^{\prime} & 0 & z_{2} \varphi_{23}^{\prime} & z_{2} \varphi_{24}^{\prime} \\
-z_{1} \varphi_{13}^{\prime} & -z_{2} \varphi_{23}^{\prime} & 0 & \varphi_{34}^{\prime} \\
-z_{1} \varphi_{14}^{\prime} & -z_{2} \varphi_{24}^{\prime} & -\varphi_{34}^{\prime} & 0
\end{array}\right)
$$

where $\varphi_{i j}^{\prime}$ depends only on $y_{3}, y_{4}$. From this we immediately conclude that

$$
\left[t_{i}, \mathfrak{D}(f)\right]=\mathfrak{D}\left(t_{i}(f)\right), \quad i=1,2 .
$$

Thus the 1 -section determined by the zero weight space of ad $t_{1}$, ad $t_{2}$ contains

$$
\operatorname{span}\left\{\mathfrak{D}\left(y_{3}^{i} y_{4}^{j}\right) \mid 0 \leq i, j \leq p-1\right\} \cap L .
$$

Now suppose that $T_{\mathfrak{N}} \neq(0)$. Then $t_{3}$ acts on $W(4 ; \underline{1}) / W(4 ; \underline{1})_{(0)} \cong \sum_{i=1}^{4} F \partial_{i}$. Lemma 5.2 shows that $F t_{3}$ maps nontrivially into $L_{(0)} / L_{(1)} \cong \mathfrak{s p}(4)$. Therefore trace $\left(\operatorname{ad}_{\mathrm{gr}_{-1}} L t_{3}\right)=0$, whence $\gamma+\delta=0$. Thus we may assume

$$
t_{3}=y_{3} \partial_{3}-y_{4} \partial_{4} \text {. }
$$

Considering eigenvalues with respect to $t_{3}$, we see that all $\tilde{\omega}_{1 i}, \tilde{\omega}_{2 i}(i=3,4)$ have constant term 0 . As $\left(\omega_{i j}\right)$ is invertible, $\tilde{\omega}_{34}$ has to have nonzero constant term. Thus

$$
\left(\omega_{i j}\right) \equiv\left(\begin{array}{cccc}
0 & \alpha & 0 & 0 \\
-\alpha & 0 & 0 & 0 \\
0 & 0 & 0 & \beta \\
0 & 0 & -\beta & 0
\end{array}\right), \quad \alpha \beta \neq 0
$$

modulo terms of degree $>0$. Then

$$
\left(\varphi_{i j}\right) \equiv\left(\begin{array}{cccc}
0 & -\alpha^{-1} & 0 & 0 \\
\alpha^{-1} & 0 & 0 & 0 \\
0 & 0 & 0 & -\beta^{-1} \\
0 & 0 & \beta^{-1} & 0
\end{array}\right)
$$

modulo terms of degree $>0$, whence $\varphi_{34}^{\prime}$ is invertible. It is now not hard to prove that the algebra generated by

$$
\mathfrak{D}\left(y_{3}^{2}\right), \mathfrak{D}\left(y_{3} y_{4}\right), \mathfrak{D}\left(y_{4}^{2}\right)
$$

is modulo its radical isomorphic to $\mathfrak{s l}(2)$. Thus the above mentioned 1-section is not solvable, a contradiction.

Finally, suppose that $T_{\mathfrak{N}}=(0)$. Then, similarly to the above, we may assume that

$$
t_{3}=z_{3} \partial_{3}, \quad z_{3}=y_{3}+1
$$

Since $\left(\omega_{i j}\right)$ is invertible, there is an index $i \neq 4$ such that $\tilde{\omega_{i 4}}$ has nonzero constant term. By symmetry we may assume that $i=3$. As in the former case, this leads to the existence of a nonsolvable 1 -section. This contradiction shows that no algebra $H(4 ; \underline{1} ; \Psi)^{(2)}$ satisfies $(A ; 3)$.

We summarize: 
Theorem 5.3. Let $L$ be simple satisfying $(A ; 3)$. Then $L$ is isomorphic to one of the algebras $H(2 ;(2,1) ; \Phi(\tau))^{(1)}$ or $S(3 ; \underline{1} ; \Phi(\tau))^{(1)}$.

We are now going to normalize all 3-dimensional tori in these algebras. It will also follow from these deliberations that the above mentioned algebras indeed satisfy $(A ; 3)$.

Proposition 5.4. Suppose $L \cong H(2 ;(2,1) ; \Phi(\tau))^{(1)}$ and $T$ is a 3-dimensional torus in the p-envelope $L_{p} \subset \operatorname{Der} L$. Then $\Gamma \cup\{0\}$ is an elementary p-group of order $p^{3}$. There is a biadditive form

$$
f:(\Gamma \cup\{0\}) \times(\Gamma \cup\{0\}) \rightarrow F
$$

and an eigenvector basis $\left(e_{\mu} \mid \mu \in \Gamma\right)$ of $L$ with respect to $T$ such that

$$
\left[e_{\lambda}, e_{\mu}\right]=f(\lambda, \mu) e_{\lambda+\mu} \text {. }
$$

Proof. Set $\tau=\left(p^{2}-1, p-1\right)$. According to [3] we have the following description of subalgebras in $W(2 ;(2,1))$ :

$$
\begin{aligned}
& H(2 ;(2,1) ; \Phi(\tau))=\sum_{|a| \geq 2,0<a \leq \tau} F\left(x^{\left(a-\epsilon_{2}\right)} D_{1}-x^{\left(a-\epsilon_{1}\right)} D_{2}\right) \\
& \quad+F\left(1-x^{(\tau)}\right) D_{1}+F\left(1-x^{(\tau)}\right) D_{2}+F x_{1}^{\left(p^{2}-1\right)} D_{2}+F x_{2}^{(p-1)} D_{1}, \\
& H(2 ;(2,1) ; \Phi(\tau))^{(1)}=\sum_{|a| \geq 2,0<a \leq \tau} F\left(x^{\left(a-\epsilon_{2}\right)} D_{1}-x^{\left(a-\epsilon_{1}\right)} D_{2}\right) \\
& \quad+F\left(1-x^{(\tau)}\right) D_{1}+F\left(1-x^{(\tau)}\right) D_{2},
\end{aligned}
$$

$\operatorname{Der} H(2 ;(2,1) ; \Phi(\tau))^{(1)}=H(2 ;(2,1) ; \Phi(\tau))+F\left(\left(1-x^{(\tau)}\right) D_{1}\right)^{p}$.

Note that

$$
E:=\left(\left(1-x^{(\tau)}\right) D_{1}\right)^{p}=D_{1}^{p}-x_{1}^{\left(p^{2}-p\right)} x_{2}^{(p-1)} D_{1}
$$

Considering $L_{p}$ as a subalgebra of $W(2 ;(2,1))_{p}$, we immediately see that

$$
L_{p} \cap W(2 ;(2,1))_{(0)} / L_{p} \cap W(2 ;(2,1))_{(1)}=L_{(0)} / L_{(1)} \cong \mathfrak{s l}(2) .
$$

Therefore

$$
\operatorname{dim} T \cap W(2 ;(2,1))_{(0)} \leq 1
$$

Suppose that $T \cap W(2 ;(2,1))_{(0)} \neq(0)$. Then $T \cap W(2 ;(2,1))_{(0)}$ constitutes a CSA in $L_{p} \cap W(2 ;(2,1))_{(0)} / L_{p} \cap W(2 ;(2,1))_{(1)} \cong \mathfrak{s l}(2)$, and the 2-dimensional module $W(2 ;(2,1)) / W(2 ;(2,1))_{(0)}$ for this $\mathfrak{s l}(2)$ is irreducible. Thus 0 is not a weight. This means that

$$
T \cap W(2 ;(2,1)) \subset T \cap W(2 ;(2,1))_{(0)} .
$$

Since $\operatorname{dim} L_{p} / L_{p} \cap W(2 ;(2,1))=1$, we obtain

$$
\operatorname{dim} T \leq \operatorname{dim} L_{p} / L_{p} \cap W(2 ;(2,1))+\operatorname{dim} T \cap W(2 ;(2,1))_{(0)}=2,
$$

a contradiction. Hence

$$
T \cap W(2 ;(2,1))_{(0)}=(0) .
$$

As a consequence, there are $E_{0}, E_{1}, E_{2} \in W(2 ;(2,1))_{(0)}$ such that

$$
T=F\left(D_{1}^{p}+E_{0}\right) \oplus F\left(D_{1}+E_{1}\right) \oplus F\left(D_{2}+E_{2}\right) .
$$

We are now going to realize $H(2 ;(2,1) ; \Phi(\tau))^{(1)}$ as a Poisson Lie algebra. Impose on the divided power algebra $A(2 ;(2,1))$ a Poisson bracket

$$
\left\{x^{(a)}, x^{(b)}\right\}:=\left(x^{\left(a-\epsilon_{1}\right)} x^{\left(b-\epsilon_{2}\right)}-x^{\left(a-\epsilon_{2}\right)} x^{\left(b-\epsilon_{1}\right)}\right)\left(1-x^{(\tau)}\right) .
$$


It is easily checked that

$$
(A(2 ;(2,1)),\{,\})^{(1)} \cap F 1=(0) .
$$

Define a linear mapping $\mathfrak{D}: A(2 ;(2,1)) \rightarrow H(2 ;(2,1) ; \Phi(\tau))$ by

$$
\begin{aligned}
\mathfrak{D}\left(x^{(a)}\right) & =x^{\left(a-\epsilon_{1}\right)} D_{2}-x^{\left(a-\epsilon_{2}\right)} D_{1} \quad \text { if }|a| \geq 2, \\
\mathfrak{D}\left(x_{1}\right) & =\left(1-x^{(\tau)}\right) D_{2}, \quad \mathfrak{D}\left(x_{2}\right)=-\left(1-x^{(\tau)}\right) D_{1}, \\
\mathfrak{D}(1) & =0 .
\end{aligned}
$$

This definition is analoguous to that mentioned in the case (H4) above, where we now have $\omega=\left(1+x^{(\tau)}\right) d x_{1} \wedge d x_{2}$ and $\varphi_{12}=-\omega_{12}{ }^{-1}=1-x^{(\tau)}$. Note that

$$
[\mathfrak{D}(f), \mathfrak{D}(g)]=\mathfrak{D}(\mathfrak{D}(f)(g))=\mathfrak{D}(\{f, g\}) \quad \forall f, g \in A(2 ;(2,1)) .
$$

Thus $\mathfrak{D}$ is a homomorphism of algebras with $\operatorname{ker}(\mathfrak{D})=F 1$, and hence $\mathfrak{D}$ establishes an isomorphism of Lie algebras

$$
A(2 ;(2,1))^{(1)} \stackrel{\sim}{\longrightarrow} H(2 ;(2,1) ; \Phi(\tau))^{(1)} .
$$

Next we tie back the action of the torus $T$. Since $L_{p} \subset W(2 ;(2,1))_{p},(1)$ implies that the action of $L_{p}$ on $A(2 ;(2,1))^{(1)}$ is given by the description of $L_{p}$ as a subalgebra in $W(2 ;(2,1))_{p}$ :

$$
\mathfrak{D}\left(\mathfrak{D}(f)^{p^{j}}(g)\right)=(\operatorname{ad} \mathfrak{D}(f))^{p^{j}}(\mathfrak{D}(g))=\left[\mathfrak{D}(f)^{p^{j}}, \mathfrak{D}(g)\right],
$$

whence

$$
\mathfrak{D}(x(g))=[x, \mathfrak{D}(g)] \quad \forall x \in L_{p} \subset W(2 ;(2,1))_{p}, \forall g \in A(2 ;(2,1)) .
$$

In particular,

$$
t(\{f, g\})=\{t(f), g\}+\{f, t(g)\} \quad \forall t \in T, \forall f, g \in A(2 ;(2,1))^{(1)} .
$$

Next we observe that there is a natural isomorphism $A(2 ;(2,1)) \stackrel{\sim}{\longrightarrow} A(3 ; \underline{1})$ of commutative algebras via

$$
x^{\left(\epsilon_{1}\right)} \mapsto y_{1}, \quad x^{\left(p \epsilon_{1}\right)} \mapsto y_{2}, \quad x^{\left(\epsilon_{2}\right)} \mapsto y_{3} .
$$

Thus $A(3 ; \underline{1})$ carries a related Poisson bracket (its form will be determined below). Moreover, $T$ is mapped into $W(3 ; \underline{1})$, it acts on $(A(3 ; \underline{1}),\{\})^{(1)}$ by derivations, and the above described form of $T$ ensures that

$$
T=F\left(\partial_{1}+e_{1}\right) \oplus F\left(\partial_{2}+e_{2}\right) \oplus F\left(\partial_{3}+e_{3}\right), \quad e_{1}, e_{2}, e_{3} \in W(3 ; \underline{1})_{(0)} .
$$

Similarly to what we have done in the previous case, there are generators $y_{1}, y_{2}, y_{3}$ of the truncated polynomial ring $A(3 ; \underline{1})$ and toral elements $t_{1}, t_{2}, t_{3} \in T$, such that

$$
t_{i}\left(y_{j}\right)=\delta_{i j}\left(y_{j}+1\right) .
$$

Set $z_{j}:=y_{j}+1$. Then with $\partial_{i}=\partial / \partial y_{i}$ we have $t_{i}=z_{i} \partial_{i}$. Considering eigenvalues, we obtain

$$
\left\{z_{i}, z_{j}\right\}=\alpha_{i j} z_{i} z_{j}, \quad \alpha_{i j} \in F .
$$

Then the Poisson bracket is given by

$$
\left\{z^{a}, z^{b}\right\}=\sum_{i, j=1}^{3} a_{i} b_{j} z^{a+b-\epsilon_{i}-\epsilon_{j}}\left\{z_{i}, z_{j}\right\}=\sum_{i, j=1}^{3} a_{i} b_{j} \alpha_{i j} z^{a+b} .
$$


We now set

$$
\begin{aligned}
G & :=\sum_{i=1}^{3} G F(p) \epsilon_{i}, \quad \Gamma=G \backslash\{0\}, \\
e_{a} & :=z^{a}, \quad f(a, b)=\sum_{i, j=1}^{3} a_{i} b_{j} \alpha_{i j} .
\end{aligned}
$$

This proves the proposition.

From this realization of $H(2 ;(2,1) ; \Phi(\tau))^{(1)}$ and $T$ one deduces immediately that $C_{H(2 ;(2,1) ; \Phi(\tau))^{(1)}}(T)=(0)$. Thus this algebra satisfies $(A ; 3)$.

Proposition 5.5. Suppose $L \cong S(3 ; \underline{1} ; \Phi(\tau))^{(1)}$ and $T$ is a 3-dimensional torus in the $p$-envelope $L_{p} \subset$ Der $L$. Then there are generators $y_{1}, y_{2}, y_{3}$ of $A(3 ; \underline{1})$ such that

$$
\begin{aligned}
& L \subset\left\{D \in W(3 ; \underline{1}) \mid D\left(\prod_{i=1}^{3}\left(y_{i}+1\right)^{p-1} d y_{1} \wedge d y_{2} \wedge d y_{3}\right)=0\right\}, \\
& T=\bigoplus_{i=1}^{3} F\left(y_{i}+1\right) \partial_{i} .
\end{aligned}
$$

Proof. Recall that (we will use here the notion of regular powers and not of divided powers)

$$
\begin{aligned}
\omega= & \left(1-x_{1}^{p-1} x_{2}^{p-1} x_{3}^{p-1}\right) d x_{1} \wedge d x_{2} \wedge d x_{3}, \\
S(3 ; \underline{1} ; \Phi(\tau))= & \{E \in W(3 ; \underline{1}) \mid E(\omega)=0\} \\
= & \sum_{i=1}^{3} F\left(1+x_{1}^{p-1} x_{2}^{p-1} x_{3}^{p-1}\right) D_{i} \\
& +\sum_{1 \leq i<j \leq 3} \sum_{0 \leq a_{\ell} \leq p-1,|a| \geq 2} F\left(a_{i} x^{a-\epsilon_{i}} D_{j}-a_{j} x^{a-\epsilon_{j}} D_{i}\right) \\
& +\sum_{i=1}^{3} F\left(\prod_{k \neq i} x_{k}^{p-1}\right) D_{i} .
\end{aligned}
$$

We may realize $L_{p}$ as a subalgebra of $W(3 ; \underline{1})$, and hence may assume that

$$
T(\omega)=0
$$

It is easily seen that

$$
L_{p} \subset L+W(3 ; \underline{1})_{(1)} .
$$

Thus we have a natural embedding

$$
T \cap W(3 ; \underline{1})_{(0)} \hookrightarrow L_{(0)} / L_{(1)} \cong \mathfrak{s l}(3) .
$$

Consequently, $\operatorname{dim} T \cap W(3 ; \underline{1})_{(0)} \leq 2$.

Suppose $\operatorname{dim} T \cap W(3 ; \underline{1})_{(0)}=2$. Then $T \cap W(3 ; \underline{1})_{(0)}$ constitutes a CSA of $\mathfrak{s l}(3) \cong L_{(0)} / L_{(1)}$. Since $L / L_{(0)}$ is the natural 3-dimensional $\mathfrak{s l}(3)$-module, 0 is not a weight. This implies that $T \subset W(3 ; \underline{1})_{(0)}$, i.e., $\operatorname{dim} T \cap W(3 ; \underline{1})_{(0)}=3$, a contradiction.

Suppose $\operatorname{dim} T \cap W(3 ; \underline{1})_{(0)}=1$, and set $T \cap W(3 ; \underline{1})_{(0)}=: F t$. Then $t$ acts trivially on $T / T \cap W(3 ; \underline{1})_{(0)}$. Since $t \notin W(3 ; \underline{1})_{(1)}$, it does not act trivially on $W(3 ; \underline{1}) / W(3 ; \underline{1})_{(0)}$. Thus $\operatorname{ad}_{W(3 ; \underline{1}) / W(3 ; \underline{1})_{(0)}} t$ has eigenvalue 0 with multiplicity 2 , 
and exactly 1 nonzero eigenvalue (with multiplicity 1$)$. As $\operatorname{trace}\left(\operatorname{ad}_{W(3 ; \underline{1}) / W(3 ; \underline{1})_{(0)}} t\right)$ $=0$, this is impossible. Consequently,

$$
T \cap W(3 ; \underline{1})_{(0)}=(0) .
$$

As in the previous cases we choose generators $y_{1}, y_{2}, y_{3}$ of the truncated polynomial ring $A(3 ; \underline{1})$ and toral elements $t_{1}, t_{2}, t_{3} \in T$ such that

$$
t_{i}\left(y_{j}\right)=\delta_{i j}\left(y_{i}+1\right), \quad 1 \leq i, j \leq 3 .
$$

We now express $\omega$ in terms of these generators. Clearly, as $\omega$ is a volume form it can be written as $\omega=g\left(y_{1}, y_{2}, y_{3}\right) d y_{1} \wedge d y_{2} \wedge d y_{3}$. Since $T$ annihilates $\omega$, we obtain

$$
\omega=\alpha \prod_{i=1}^{3}\left(y_{i}+1\right)^{p-1} d y_{1} \wedge d y_{2} \wedge d y_{3}, \quad \alpha \neq 0 .
$$

We are now able to derive necessary information on sections. Write, as above,

$$
\begin{aligned}
& z_{i}=y_{i}+1, \quad i=1,2,3, \quad \text { and observe that } z_{i}^{p}=1, \\
& \omega=\prod_{i=1}^{3} z_{i}^{p-1} d y_{1} \wedge d y_{2} \wedge d y_{3}, \\
& T=\sum F z_{i} \partial_{i} \quad \text { with } \partial_{i}=\partial / \partial y_{i}, \\
& S(3 ; \underline{1} ; \Phi(\tau))=\{D \in W(3 ; \underline{1}) \mid D(\omega)=0\} .
\end{aligned}
$$

Every element of $W(3 ; \underline{1})$ has the form $\sum f_{i} z_{i} \partial_{i}\left(\right.$ as $\left.z_{i}^{-1}=z_{i}^{p-1}\right)$, and clearly

$$
\sum f_{i} z_{i} \partial_{i} \in S(3 ; \underline{1} ; \Phi(\tau)) \Longleftrightarrow \sum z_{i} \partial_{i}\left(f_{i}\right)=0 .
$$

Thus if $\alpha=\sum a_{i} \epsilon_{i} \neq 0$, then the corresponding root space

$$
S(3 ; \underline{1} ; \Phi(\tau))_{\alpha}=\left\{\sum_{i=1}^{3} \lambda_{i} z^{a} z_{i} \partial_{i} \mid \sum_{i=1}^{3} \lambda_{i} a_{i}=0\right\}
$$

is 2-dimensional, while

$$
S(3 ; \underline{1} ; \Phi(\tau))_{0}=T
$$

is 3-dimensional. Note that

$$
\left[\sum \lambda_{j} z^{a} z_{j} \partial_{j}, \sum \mu_{i} z^{b} z_{i} \partial_{i}\right\}=\sum_{i, j}\left(\lambda_{j} b_{j} \mu_{i}-\mu_{j} a_{j} \lambda_{i}\right) z^{a+b} z_{i} \partial_{i} .
$$

Thus if

$$
E_{1}=\sum \lambda_{i} z^{a} z_{i} \partial_{i} \in S(3 ; \underline{1} ; \Phi(\tau))_{\alpha}, \quad E_{2}=\sum \mu_{i} z^{b} z_{i} \partial_{i} \in S(3 ; \underline{1} ; \Phi(\tau))_{k \alpha}
$$

for $k \neq 0$, then, setting $\alpha=\sum a_{i} \epsilon_{i}, k \alpha=\sum b_{i} \epsilon_{i}$, one has

$$
b_{i}=k a_{i}, \sum \lambda_{j} b_{j}=k \sum \lambda_{j} a_{j}=0, \sum \mu_{j} a_{j}=k^{-1} \sum \mu_{j} b_{j}=0 .
$$

Consequently, (2) shows that $\left[E_{1}, E_{2}\right]=(0)$. Thus every 1 -section is abelian. In particular,

$$
T \cap S(3 ; \underline{1} ; \Phi(\tau))^{(1)}=(0) .
$$

This observation shows that $S(3 ; \underline{1} ; \Phi(\tau))^{(1)}$ in fact satisfies $(A ; 3)$.

Theorem 5.6. Suppose that $L$ satisfies $(A ; 3)$ and $T$ is a 3 -dimensional torus in a semisimple p-envelope of $L$. Then:

1) No root vector acts nilpotently on $L$.

2) Every solvable 2-section of $L$ is abelian. 
3) $\Gamma \cup\{0\}$ is an elementary $p$-group of order $p^{3}$.

4) For $x \in L_{\alpha}, y \in L_{\beta}$ one has

$$
([x, y])^{[p]}=-\alpha\left(y^{[p]}\right) x^{[p]}+\beta\left(x^{[p]}\right) y^{[p]} .
$$

Proof. (a) Suppose $L \cong H(2 ;(2,1) ; \Phi(\tau))^{(1)}$. With the notations of Proposition 5.4 we have $f(\lambda, \mu+k \lambda)=f(\lambda, \mu)$ for $k \in G F(p)$, and hence

$$
\left(\operatorname{ad} e_{\lambda}\right)^{p}\left(e_{\mu}\right)=f(\lambda, \mu)^{p} e_{\mu} .
$$

Since $L$ is centerless, for every given $\lambda$ there is $\mu$ with $f(\lambda, \mu) \neq 0$. Thus ad $e_{\lambda}$ is not nilpotent.

Next let $L(\lambda, \mu)$ be a 2 -section. If $f(\lambda, \mu)=0$ then $L(\lambda, \mu)$ is abelian, while in case $f(\lambda, \mu) \neq 0$ we conclude from $(3)$ that

$$
e_{\mu}=f(\lambda, \mu)^{-p}\left(\operatorname{ad} e_{\lambda}\right)^{p}\left(e_{\mu}\right), \quad e_{\lambda}=-f(\lambda, \mu)^{-p}\left(\operatorname{ad} e_{\mu}\right)^{p}\left(e_{\lambda}\right) .
$$

Then $L(\lambda, \mu)$ is nonsolvable.

$3)$ is stated in Proposition 5.4.

Finally, for $x=e_{\alpha}, y=e_{\beta}$ one has

$$
\begin{aligned}
\left(\operatorname{ad}\left[e_{\alpha}, e_{\beta}\right]\right)^{p}\left(e_{\mu}\right) & =f(\alpha, \beta)^{p}\left(\operatorname{ad} e_{\alpha+\beta}\right)^{p}\left(e_{\mu}\right) \\
& =f(\alpha, \beta)^{p} f(\alpha+\beta, \mu)^{p} e_{\mu} \\
& =f(\alpha, \beta)^{p} f(\alpha, \mu)^{p} e_{\mu}+f(\alpha, \beta)^{p} f(\beta, \mu)^{p} e_{\mu} .
\end{aligned}
$$

As

$$
\begin{aligned}
& \beta\left(e_{\alpha}^{[p]}\right) e_{\beta}=\left(\operatorname{ad} e_{\alpha}\right)^{p}\left(e_{\beta}\right)=f(\alpha, \beta)^{p} e_{\beta}, \\
& \alpha\left(e_{\beta}^{[p]}\right) e_{\alpha}=\left(\operatorname{ad} e_{\beta}\right)^{p}\left(e_{\alpha}\right)=f(\beta, \alpha)^{p} e_{\alpha}=-f(\alpha, \beta)^{p} e_{\alpha},
\end{aligned}
$$

we obtain that

$$
\left(\left[e_{\alpha}, e_{\beta}\right]\right)^{[p]}+\alpha\left(e_{\beta}^{[p]}\right) e_{\alpha}^{[p]}-\beta\left(e_{\alpha}^{[p]}\right) e_{\beta}^{[p]}
$$

centralizes $L$, and therefore vanishes. This proves 3$)$.

(b) Suppose $L \cong S(3 ; \underline{1} ; \Phi(\tau))^{(1)}$. With the notations of Proposition 5.5 we have, for every root vector $E_{a, \lambda}:=\sum \lambda_{i} z^{a} z_{i} \partial_{i}$ and $k \in G F(p)\left(\right.$ as $\left.\sum \lambda_{i} a_{i}=0\right)$,

$$
E_{a, \lambda}\left(z^{b+k a}\right)=\sum \lambda_{i}\left(b_{i}+k a_{i}\right) z^{b+(k+1) a}=\left(\sum \lambda_{i} b_{i}\right) z^{b+(k+1) a} .
$$

Thus $E_{a, \lambda}^{[p]}\left(z^{b}\right)=\left(\sum \lambda_{i} b_{i}\right)^{p} z^{b}=\sum \lambda_{i}^{p} b_{i} z^{b}$, i.e.,

$$
E_{a, \lambda}^{[p]}=\sum \lambda_{i}^{p} z_{i} \partial_{i} .
$$

This proves 1).

2) For any root $\alpha=\sum a_{i} \epsilon_{i} \neq 0$ the root space is 2-dimensional:

$$
L_{\alpha}=F E_{a, \lambda} \oplus F E_{a, \mu}, \text { where } \sum \lambda_{i} a_{i}=\sum \mu_{i} a_{i}=0,
$$

and hence $\operatorname{dim} L_{\alpha}{ }^{[p]}=2$. Thus $L_{\alpha}{ }^{[p]}=T \cap$ ker $\alpha$. Therefore no 2-section is solvable.

$3)$ is obvious.

4) Set $\alpha=\sum a_{i} \epsilon_{i}, \beta=\sum b_{i} \epsilon_{i}$ and $x=E_{a, \lambda}, y=E_{b, \mu}$. Then, according to (2),

$$
[x, y]=\sum_{i, j}\left(\lambda_{j} b_{j} \mu_{i}-\mu_{j} a_{j} \lambda_{i}\right) z^{a+b} z_{i} \partial_{i}
$$


and hence, according to (4)

$$
\begin{aligned}
x^{[p]} & =\sum \lambda_{j}^{p} z_{j} \partial_{j}, \quad \beta\left(x^{[p]}\right)=\sum \lambda_{j}^{p} b_{j}, \\
y^{[p]} & =\sum \mu_{j}^{p} z_{j} \partial_{j}, \quad \alpha\left(y^{[p]}\right)=\sum \mu_{j}^{p} a_{j}, \\
{[x, y]^{[p]} } & =\sum_{i}\left(\sum_{j} \lambda_{j} b_{j} \mu_{i}-\mu_{j} a_{j} \lambda_{i}\right)^{p} z_{i} \partial_{i} \\
& =-\sum_{i}\left(\sum_{j} \mu_{j}^{p} a_{j}\right) \lambda_{i}^{p} z_{i} \partial_{i}+\sum_{i}\left(\sum_{j} \lambda_{j}^{p} b_{j}\right) \mu_{i}^{p} z_{i} \partial_{i} .
\end{aligned}
$$

This proves 4).

\section{Solvable Sections}

We now turn to the general case that $L$ is a simple Lie algebra over an algebraically closed field $F$ of characteristic $p>7$ satisfying $(A ; m)$ for some $m$. Let $T$ be a torus of a $p$-envelope $L_{p}$ of $L$ of maximal absolute toral rank. We recall from $\S 1$ that $C_{L}(T)$ acts nilpotently on $L$ and every 1 -section is nilpotent.

We quote (with minor changes)

Theorem 6.1 ([17, (2.7)]). Let $L$ be simple satisfying $(A ; T R(L))$ and let $L_{p}$ be a semisimple p-envelope of $L$. Let $T$ denote a torus of dimension $\operatorname{dim} T=T R(L)$. Let $I(\alpha, \beta, \gamma)$ be the maximal solvable ideal of $L(\alpha, \beta, \gamma)+T$ and $\sigma: L(\alpha, \beta, \gamma)+T \rightarrow$ $L(\alpha, \beta, \gamma)+T / I(\alpha, \beta, \gamma)$. Put $R=\sigma(T)$ and $K=\sigma(L(\alpha, \beta, \gamma))$. Then $K$ is one of the following:

1) $K=(0)$;

2) $S \subset K+R \subset \operatorname{Der} S, S \cong H\left(2 ; \underline{1} ; \Phi(\tau)^{(1)}\right.$, and there exist $\delta_{i}(i=1,2)$ such that $K=\sigma\left(L\left(\delta_{1}, \delta_{2}\right)\right)$;

3) $H(2 ; \underline{1} ; \Phi(\tau))^{(1)} \otimes A(1 ; \underline{1}) \subset K+R \subset \operatorname{Der}\left(H(2 ; \underline{1} ; \Phi(\tau))^{(1)} \otimes A(1 ; \underline{1})\right)$;

4) $S \subset K+R \subset \operatorname{Der} S$, where $S$ is a simple Lie algebra satisfying $(A ; 3)$.

We are going to derive some detailed information on these cases.

Lemma 6.2. Suppose that $L(\alpha, \beta, \gamma)$ satisfies case 2) of Theorem 6.1. Then the following hold.

1) $K=S$.

2) $\operatorname{dim} R=2$.

3) Every root space of $K$ for a nonzero root is 1-dimensional, and $C_{K}(R)=(0)$. Every root vector acts nonnilpotently on $K$.

4) $K+R=\operatorname{Der} S$.

Proof. Since $K$ is the image of a 2-section, we may apply [14, (VII.4)] to obtain $K \cong H\left(2 ; \underline{1} ; \Phi(\tau)^{(1)}\right.$. Thus $K=S$. In addition, as it cannot be an image of a (solvable!) 1 -section, we have $1<\operatorname{dim} R \leq 2$. 3) is an immediate consequence of [14, (VII. 3)]. As a consequence of 3$)$ we obtain $\operatorname{dim}(K+R)=\operatorname{dim} K+\operatorname{dim} R=$ $p^{2}+1=\operatorname{dim} \operatorname{Der} H(2 ; \underline{1} ; \Phi(\tau))^{(1)}$.

Lemma 6.3. Suppose that $L(\alpha, \beta, \gamma)$ is as in case 3) of Theorem 6.1. Set $S:=$ $H(2 ; \underline{1} ; \Phi(\tau))^{(1)} \otimes A(1 ; \underline{1})$. Then the following hold.

1) $K / S$ is solvable.

2) $\operatorname{dim} R=3$, i.e., there are $3 F$-independent roots on $S$. 
3) ker $\sigma$ acts nilpotently on $L(\alpha, \beta, \gamma)$.

4) There is $\kappa \in\langle\alpha, \beta, \gamma\rangle, \kappa \neq 0$, satisfying $\kappa\left(K_{\mu}\right)=0$ for all $\mu \in\langle\alpha, \beta, \gamma\rangle$.

Proof. 1) Note that

$$
\begin{gathered}
T R(S)=T R\left(H(2 ; \underline{1} ; \Phi(\tau))^{(1)} \otimes A(1 ; \underline{1}) / H(2 ; \underline{1} ; \Phi(\tau))^{(1)} \otimes A(1 ; \underline{1})_{(1)}\right) \\
=T R\left(H(2 ; \underline{1} ; \Phi(\tau))^{(1)}\right)=2 .
\end{gathered}
$$

Therefore $R$ intersects the $p$-envelope $S_{p}$ in Der $S$ in a 2-dimensional torus $R_{0}$ ([17, (1.5)]). Thus there is a root $\lambda$ satisfying

$$
K=S+K(\lambda), \quad \lambda\left(R_{0}\right)=0 .
$$

Consequently, $\bigcap_{n>0} K^{(n)}=S$. This proves 1$)$.

2) We assume $\operatorname{dim} R \leq 2$. Then we have $R=R_{0}, K(\lambda)=C_{K}(R)=\sigma\left(C_{L}(T)\right)$. Let $J$ denote the unique maximal ideal of $S$. Observe that $J$ is nilpotent. Now $J+\left[C_{K}(R), J\right]$ is an ideal of $S$. If this is contained in $J$, then $J$ is a solvable ideal of $K+R$. By construction of $K$ this has to be (0), which is not true. Thus we have $S=J+\left[C_{K}(R), J\right]$. Due to our assumption, $J$ decomposes with respect to $R_{0}=R$. Then for every root $\mu$

$$
S_{\mu}=J_{\mu}+\left[C_{K}(R), J_{\mu}\right] \subset J_{\mu}+(K(\mu))^{(1)} \cap S .
$$

As $L(\mu)$ is a nilpotent and hence triangulable [11, Theorem 3.5]) section, therefore every root space $S_{\mu}$ acts nilpotently on $S$ (by Jacobson's theorem on nil Lie sets). This would imply the nilpotency of $S$, a contradiction. Since $R$ acts faithfully on $S$, there are $3 F$-independent roots on $S$.

3) Let $x \in \operatorname{ker} \sigma$ be any root rector. We may assume that $\alpha, \beta, \gamma$ are independent roots on $S$. According to Proposition 1.1, $\alpha(x)=\beta(x)=\gamma(x)=0$. Then $\mu(x)=0$ holds for all $\mu \in\langle\alpha, \beta, \gamma\rangle$, whence $x$ acts nilpotently on $L(\alpha, \beta, \gamma)$. Thus $\operatorname{ker} \sigma$ acts nilpotently as well.

4) As in 2), let $R_{0}$ denote the 2-dimensional torus $R_{0}=R \cap S_{p}$. There is $\kappa \in\langle\alpha, \beta, \gamma\rangle$ satisfying $\kappa\left(R_{0}\right)=0, \kappa \neq 0$. Choose roots $\delta_{1}, \delta_{2} F$-independent on $R_{0}$. We observe that $S / \operatorname{rad} S \cong H(2 ; \underline{1} ; \Phi(\tau))^{(1)}$, and hence $\sigma\left(L\left(\delta_{1}, \delta_{2}\right)\right)$ is of type $H(2 ; \underline{1} ; \Phi(\tau))^{(1)}$ as well. Consequently, $\delta_{1}\left(L_{\delta_{2}}\right) \neq 0, \delta_{2}\left(L_{\delta_{1}}\right) \neq 0$ ([14, (VII.3)]). Now $\kappa$ satisfies

$$
\kappa\left(\bigcup_{\mu} S_{\mu}\right)=\kappa\left(R_{0}\right)=0
$$

As in 1), decompose

$$
K=S+K(\lambda), \quad \lambda\left(R_{0}\right)=0,
$$

and observe that, due to the preceding result, it remains to prove that $\kappa\left(K_{i \lambda}\right)=0$ for all $i \in G F(p)$. If $\lambda=0$, i.e., $K=S+\sigma\left(C_{L}(T)\right)$, then $\kappa\left(K_{i \lambda}\right)=0$, since every root vanishes on $C_{L}(T)$. Otherwise we have $\kappa=s \lambda$ for some $s \in F$, and hence $\kappa\left(K_{i \lambda}\right)=0$ as well.

Lemma 6.4. Suppose that $L(\alpha, \beta, \gamma)$ satisfies case 4) of Theorem 6.1. Then

1) $\operatorname{dim} R=3$,

2) $K=S+C_{K}(R)$.

Proof. As $T R(S)=3, R$ intersects the $p$-envelope $S_{p}$ in Der $S$ in a 3-dimensional torus [17, (1.5)], i.e., $\operatorname{dim} R=3$ and $R \subset S_{p}$. The latter implies $K=S+C_{K}(R)$. 
With $\gamma=\alpha$ Theorem 6.1 also covers the case of 2 -sections. According to Lemmas 6.3 and 6.4, only cases 1) and 2) are possible then. Lemmas 6.2 and 6.4 in combination with Theorem 5.6 and the results of $[14, \mathrm{VII}]$ enable us to deal with cases 2) and 4) of Theorem 6.1. The following lemma will help us to overcome the difficulties arising from cases 1 ) and 3 ).

Lemma 6.5. Let $\alpha, \beta \in \Gamma, \gamma, \delta \in\langle\alpha, \beta\rangle$, and $V \subset L_{\gamma-\delta}$ a subspace such that

a) $\beta\left(L_{\alpha}\right) \neq 0$,

b) $\Omega:=\left\{\mu \in \Gamma \mid \mu\left(\left[V, L_{\delta}\right]\right) \neq 0\right\} \neq \emptyset$.

Then there is a root $\kappa \in \Gamma$ such that

$$
\kappa\left(\left[V, L_{\delta}\right]\right) \neq 0,(\alpha-\kappa)\left(\left[V, L_{\delta}\right]\right) \neq 0, \beta\left(\left[L_{\alpha-\kappa}, L_{\kappa}\right]\right) \neq 0 .
$$

Let $\sigma: L(\alpha, \beta, \kappa)+T \rightarrow K+R$ and $S$ be as in Theorem 6.1. The following are true.

1) $L_{\kappa}, L_{\alpha-\kappa},\left[V, L_{\delta}\right]$ and $L_{\mu}$ (if $\mu \in\langle\alpha, \beta, \kappa\rangle$ and $\mu\left(\left[L_{\alpha-\kappa}, L_{\kappa}\right]\right) \neq 0$ ) are contained in $\bigcap_{n>0} L(\alpha, \beta, \kappa)^{(n)}$.

2) $K_{\kappa}, K_{\alpha-\kappa}, \sigma\left(\left[V, L_{\delta}\right]\right)$ are nonzero subspaces contained in $S$.

3) $L(\alpha, \beta, \kappa)$ does not satisfy case 1) of Theorem 6.1. It satisfies case 2) of Theorem 6.1 if and only if $\kappa \in\langle\alpha, \beta\rangle$.

4) $S=\bigcap_{n>0} K^{(n)}$.

Proof. The simplicity of $L$ implies (by Schue's Lemma)

$$
L_{\alpha}=\sum_{\alpha-\mu, \mu \in \Omega}\left[L_{\alpha-\mu}, L_{\mu}\right] .
$$

Since $L(\alpha)$ acts triangulably, every root is linear on $L_{\alpha}$. Thus there is $\kappa \in \Gamma$ such that

$$
\kappa\left(\left[V, L_{\delta}\right]\right) \neq 0, \quad(\alpha-\kappa)\left(\left[V, L_{\delta}\right]\right) \neq 0, \beta\left(\left[L_{\alpha-\kappa}, L_{\kappa}\right]\right) \neq 0 .
$$

1) Suppose inductively (for $\mu \in\langle\alpha, \beta, \kappa\rangle$ and $\mu\left(\left[L_{\alpha-\kappa}, L_{\kappa}\right]\right) \neq 0$ ) that

$$
L_{\kappa}, L_{\alpha-\kappa},\left[V, L_{\delta}\right], L_{\mu} \subset L(\alpha, \beta, \kappa)^{(n)} .
$$

Note that $\gamma-\delta, \delta$ have to be independent, since by assumption $L(\gamma-\delta, \delta)^{(1)}$ acts non-nilpotently. Thus $\gamma-\delta$ or $\delta$ is of the form $i \alpha+j \beta$ with $j \neq 0$. Consequently

$$
(\gamma-\delta)\left(\left[L_{\alpha-\kappa}, L_{\kappa}\right]\right) \neq 0 \quad \text { or } \quad \delta\left(\left[L_{\alpha-\kappa}, L_{\kappa}\right]\right) \neq 0 .
$$

By Proposition 1.2 we see that $L_{\gamma-\delta} \subset L(\alpha, \beta, \kappa)^{(n+1)}$ or $L_{\delta} \subset L(\alpha, \beta, \kappa)^{(n+1)}$. Thus $\left[V, L_{\delta}\right] \subset L(\alpha, \beta, \kappa)^{(n+1)}$ in either case. The same reasoning then yields

$$
L_{\kappa}, L_{\alpha-\kappa} \subset L(\alpha, \beta, \kappa)^{(n+1)} \text { and } L_{\mu} \subset L(\alpha, \beta, \kappa)^{(n+1)} .
$$

In particular, $L(\alpha, \beta, \kappa)$ is nonsolvable.

2) If $\left[V, L_{\delta}\right]$ is contained in $\operatorname{ker} \sigma$, then we may conclude as above that $\left[V, L_{\sigma}\right] \subset$ $\bigcap_{n>0}(\operatorname{ker} \sigma)^{(n)}=(0)$, a contradiction. Similarly, we obtain $\sigma\left(L_{\kappa}\right) \neq 0, \sigma\left(L_{\alpha-\kappa}\right) \neq$ 0 .

3) We have already mentioned that $L(\alpha, \beta, \kappa)$ is nonsolvable. Suppose $L(\alpha, \beta, \kappa)$ satisfies case 2) of Theorem 6.1. Then $K=S \cong H(2 ; \underline{1} ; \Phi(\tau))^{(1)}$ and $\operatorname{dim} R=2$ (Lemma 6.2). Since $0 \neq \sigma\left(\left[V, L_{\delta}\right]\right) \subset\left[K_{\gamma-\delta}, K_{\delta}\right]$, then $\gamma$ and $\delta$ are independent roots on $K$, and hence span the roots lattice. As $\kappa$ is a root on $K$, we obtain $\kappa \in\langle\gamma, \delta\rangle=\langle\alpha, \beta\rangle$. 
If $\kappa \in\langle\alpha, \beta\rangle$, then $L(\alpha, \beta, \kappa)$ is a 2-section. Hence $R$ is 2-dimensional and $T R(L(\alpha, \beta, \kappa)) \leq 2$. According to Lemma $6.6, L(\alpha, \beta, \kappa)$ cannot be as in case 3 ) of Theorem 6.1. It cannot be as in case 4) of Theorem 6.1 , since otherwise $\operatorname{TR}(L(\alpha, \beta, \kappa))=3$.

4) If we are in case 2) of Theorem 6.1, apply Theorem 6.2(1). Suppose that $L(\alpha, \beta, \kappa)$ satisfies case 3$)$ or case 4$)$. In both cases we have $S=\bigcap_{n>0} K^{(n)}$, as $K / S$ is solvable (Lemmas 6.3 and 6.4 ).

We are now going to determine solvable sections of $L$.

Proposition 6.6. Every solvable section $L\left(\alpha_{1}, \ldots, \alpha_{t}\right)$ acts triangulably on $L$.

Proof. Since

$$
L\left(\alpha_{1}, \ldots, \alpha_{t}\right)^{(1)}=\sum_{\gamma, \delta \in\left\langle\alpha_{1}, \ldots, \alpha_{t}\right\rangle}\left[L_{\gamma}, L_{\delta}\right]
$$

and $\bigcup_{\gamma, \delta \in\left\langle\alpha_{1}, \ldots, \alpha_{t}\right\rangle}\left[L_{\gamma}, L_{\delta}\right]$ is a Lie set, it is sufficient to prove that every 2-section $L(\alpha, \beta)$ acts triangulably on $L$.

If $\gamma\left(L_{\delta}\right)=0$ for all $\gamma, \delta \in\langle\alpha, \beta\rangle$, then every $L_{\delta}$ acts nilpotently on $L(\alpha, \beta)$ and hence $L(\alpha, \beta)$ is a nilpotent section. Consequently it acts triangulably on $L$.

Otherwise we may assume $\beta\left(L_{\alpha}\right) \neq 0$. If $L(\alpha, \beta)^{(1)}$ does not act nilpotently on $L$, then there are $\gamma, \delta \in\langle\alpha, \beta\rangle$ such that $\left[L_{\gamma-\delta}, L_{\delta}\right]$ acts non-nilpotently on $L$. Note that $\gamma$ and $\delta$ are independent, in particular $\neq 0$. Put

$$
\Omega:=\left\{\mu \in \Gamma \mid \mu\left(\left[L_{\gamma-\delta}, L_{\delta}\right]\right) \neq 0\right\} .
$$

The present assumption yields $\Omega \neq \emptyset$. Choose a root $\kappa$ according to Lemma 6.5. This lemma yields the following results.

a) $L(\alpha, \beta, \kappa)$ is not solvable.

b) If this section satisfies case 2 ) of Theorem 6.1 then we have $\kappa \in\langle\alpha, \beta\rangle$ and $\sigma(L(\alpha, \beta, \kappa))=\sigma(L(\alpha, \beta))$ is solvable, a contradiction.

c) Suppose $L(\alpha, \beta, \kappa)$ satisfies case 3$)$ of Theorem 6.1 . Note that according to Lemma 6.5, $(i \alpha+j \beta)\left(\left[L_{\alpha-\kappa}, L_{\kappa}\right]\right)=j \beta\left(\left[L_{\alpha-\kappa}, L_{\kappa}\right]\right) \neq 0$ if $j \neq 0$. Therefore $L_{i \alpha+j \beta} \subset \bigcap_{n>0} L(\alpha, \beta, \kappa)^{(n)}$, and hence

$$
K_{i \alpha+j \beta}=\sigma\left(L_{i \alpha+j \beta}\right) \subset S \quad \forall j \neq 0 .
$$

If $\gamma \in G F(p) \alpha$ then, as $\gamma, \delta$ are independent, $\delta \notin G F(p) \alpha$, and the above shows that $K_{\gamma-\delta}, K_{\delta} \subset S$.

If $\gamma \notin G F(p) \alpha$ then $\gamma=i \alpha+j \beta$ for some $j \neq 0$, and the above shows that $K_{\gamma} \subset S$. Since according to Lemma $6.5(2) K_{\kappa}, K_{\alpha-\kappa},\left[K_{\gamma-\delta}, K_{\delta}\right] \subset S$ and $\gamma\left(\left[K_{\kappa}, K_{\alpha-\kappa}\right]\right) \neq$ 0 , Lemma 1.2 yields

$$
\left[K_{\gamma-\delta}, K_{\delta}\right]=\left(\operatorname{ad}\left(\left[K_{\kappa}, K_{\alpha-\kappa}\right]\right)\right)^{p}\left(\left[K_{\gamma-\delta}, K_{\delta}\right]\right) \subset S(\alpha, \beta)^{(1)} .
$$

In either case we obtain $\left[K_{\gamma-\delta}, K_{\delta}\right] \subset S(\alpha, \beta)^{(1)}$.

Recall that $S=H(2 ; \underline{1} ; \Phi(\tau))^{(1)} \otimes A(1 ; \underline{1})$. As $T R(S)=2, R$ intersects the $p$-envelope $S_{p}$ in Der $S$ in a 2-dimensional torus $R_{0}$. Let

$$
\pi: S+R_{0} \rightarrow \operatorname{Der} H(2 ; \underline{1} ; \Phi(\tau))^{(1)}
$$

denote the canonical homomorphism. $\pi\left(R_{0}\right)$ acts on $\pi(S)$ faithfully as a 2-dimensional torus, and $\pi(S(\alpha, \beta))$ is solvable due to the general assumption. Then 
$\pi(S(\alpha, \beta))$ can only be a 1 -section with respect to $\pi\left(R_{0}\right)$, and as all 1 -sections in $H(2 ; \underline{1} ; \Phi(\tau))^{(1)}$ are abelian $([14,($ VII.3)]), we obtain

$$
S(\alpha, \beta)^{(1)} \subset \operatorname{ker} \pi=H(2 ; \underline{1} ; \Phi(\tau))^{(1)} \otimes A(1 ; \underline{1})_{(1)} .
$$

Then $S(\alpha, \beta)^{(1)}$ acts nilpotently on $K$. Since $\kappa$ is a root on $K$, this yields the contradiction $\kappa\left(\left[L_{\gamma-\delta}, L_{\delta}\right]\right)=0$.

d) We end up with case 4) of Theorem 6.1. Then Lemma 6.4 yields $K=$ $S+C_{K}(R)$, and we obtain that

$$
K(\alpha, \beta)^{(1)} \subset S(\alpha, \beta)^{(1)}+\sum_{\mu \in\langle\alpha, \beta\rangle} K(\mu)^{(1)} .
$$

Recall that $S$ satisfies $(A ; 3)$. Then Theorem 5.6 shows that, as $S(\alpha, \beta)$ is a solvable section, $S(\alpha, \beta)$ is abelian. Therefore $K(\alpha, \beta)^{(1)}$ acts nilpotently on $K$. Since in the present case there are 3 independent roots on $S$, we obtain $\mu\left(K(\alpha, \beta)^{(1)} \cap K_{\lambda}\right)=0$ for all $\mu \in\langle\alpha, \beta, \kappa\rangle, \lambda \in\langle\alpha, \beta\rangle$. This again contradicts the choice of $\kappa$.

It has turned out during the classification work that, whenever a Lie-type theorem like the preceding proposition is proved, we are able to classify a further big family of simple Lie algebras. Accordingly, Proposition 6.6 is a key result.

Proposition 6.7. Every root vector of $L$ with respect to $T$ acts non-nilpotently on L. In particular, $C_{L}(T)=(0)$.

Proof. Put $N_{\mu}:=\left\{x \in L_{\mu} \mid \kappa(x)=0 \forall \kappa \in \Gamma\right\}$ for all $\mu \in \Gamma$. We shall prove that $N:=\sum N_{\mu}$ is an ideal of $L$.

Clearly $L(\mu)^{(1)} \subset N$ holds for all $\mu \in \Gamma$.

If $L(\alpha, \beta)$ is a solvable 2-section, then Proposition 6.6 yields

$$
\left[N_{\alpha}, L_{\beta}\right] \subset L(\alpha, \beta)^{(1)} \subset N
$$

Next suppose that $\alpha, \beta$ are independent roots and that $L(\alpha, \beta)$ is a nonsolvable 2 -section. Suppose that $\left[N_{\alpha}, L_{\beta}\right] \not \subset N$. Note that 2 -sections are described by case 2 ) of Theorem 6.1. Thus we have $\sigma(L(\alpha, \beta)) \cong H(2 ; \underline{1} ; \Phi(\tau))^{(1)}$. No root vector in this algebra acts nilpotently ([14, VII.3]). Therefore

$$
\beta\left(L_{\alpha}\right) \neq 0, \quad \alpha\left(L_{\beta}\right) \neq 0 .
$$

The assumption $\left[N_{\alpha}, L_{\beta}\right] \not \subset N$ means $\Omega:=\left\{\mu \in \Gamma \mid \mu\left(\left[N_{\alpha}, L_{\beta}\right]\right) \neq 0\right\}$ is nonvoid. Thus Lemma 6.5 applies. There is a root $\kappa$ such that

$$
\kappa\left(\left[N_{\alpha}, L_{\beta}\right]\right) \neq 0, \quad(\alpha-\kappa)\left(\left[N_{\alpha}, L_{\beta}\right]\right) \neq 0, \quad \beta\left(\left[L_{\alpha-\kappa}, L_{\kappa}\right]\right) \neq 0 .
$$

Lemma 6.5 yields the following:

a) $L(\alpha, \beta, \kappa)$ is not solvable.

b) Suppose that $L(\alpha, \beta, \kappa)$ satisfies case 2) of Theorem 6.1. Then $\kappa \in\langle\alpha, \beta\rangle$. Since in $H\left(2 ; \underline{1} ; \Phi(\tau)^{(1)}\right.$ no root vector acts nilpotently, we conclude that $\sigma\left(N_{\alpha}\right)=$ (0) and $\sigma\left(\left[N_{\alpha}, L_{\beta}\right]\right)=(0)$, contradicting Lemma 6.5(2).

c) Suppose that $L(\alpha, \beta, \kappa)$ satisfies case 3$)$ of Theorem 6.1. Lemma 6.5 implies that $\sigma\left(L_{\beta}\right) \subset \bigcap_{n>0} K^{(n)}=S$. Recalling that $\alpha\left(L_{\beta}\right) \neq 0$, we see that $\sigma\left(N_{\alpha}\right) \subset S$ as well.

Consider the homomorphism

$$
\pi: S \rightarrow S / \operatorname{rad} S \cong H(2 ; \underline{1} ; \Phi(\tau))^{(1)} .
$$


As in the proof of Proposition 6.6, let $R_{0} \subset R$ denote the 2-dimensional torus in the $p$-envelope of S. $R_{0}$ acts on $\pi(S)$ faithfully. $\pi \sigma\left(N_{\alpha}\right)$ is contained in a root space with respect to $R_{0}$. Recall that no root vector of $H(2 ; \underline{1} ; \Phi(\tau))^{(1)}$ acts nilpotently. Thus $\pi \sigma\left(N_{\alpha}\right)=(0)$. We conclude that

$$
\sigma\left(N_{\alpha}\right) \subset \operatorname{rad} S, \quad \sigma\left(\left[N_{\alpha}, L_{\beta}\right]\right)=\left[\sigma\left(N_{\alpha}\right), \sigma\left(L_{\beta}\right)\right] \subset \operatorname{rad} S .
$$

Hence $\sigma\left(\left[N_{\alpha}, L_{\beta}\right]\right)$ acts nilpotently on $S$. Since $S_{\kappa} \neq 0$ (Lemma $\left.6.5(2)\right)$, this yields the contradiction $\kappa\left(\left[N_{\alpha}, L_{\beta}\right]\right)=0$.

d) Suppose that $L(\alpha, \beta, \kappa)$ satisfies case 4$)$ of Theorem 6.1. According to Lemma 6.4 we have $K=S+C_{K}(R)$, whence $\sigma\left(N_{\alpha}\right), \sigma\left(L_{\beta}\right) \subset S$. Now $S$ satisfies $(A ; 3)$. Then Theorem 5.6 shows that $\sigma\left(N_{\alpha}\right)$ vanishes. Consequently, $\left[N_{\alpha}, L_{\beta}\right] \subset \operatorname{ker} \sigma$. As $\kappa$ is a root on $K$ (Lemma $6.5(2))$, this yields $\kappa\left(\left[N_{\alpha}, L_{\beta}\right]\right)=0$, contradicting the choice of $\kappa$.

As a consequence, $N$ is an ideal of $L$. Hence $N=(0)$, and therefore for any root vector $u \in L_{\alpha}$ there exists $\beta \in \Gamma$ such that $\beta(u) \neq 0$. Thus $u$ acts non-nilpotently on $L$. In particular $C_{L}(T) \subset N=(0)$ (cf. Theorem 1.5).

Corollary 6.8. Every solvable section is abelian, and every nonsolvable 2-section has core $H(2 ; \underline{1} ; \Phi(\tau))^{(1)}$.

Proof. This corollary is a direct consequence of Propositions 6.6 and 6.7, and Theorem 1.5.

Corollary 6.9. 1) $0 \notin \Gamma$.

2) $\Gamma \cup\{0\}$ is an elementary p-group of rank $T R(L)$.

3) There exists $k>0$ such that

$$
\operatorname{dim} L_{\mu}=k \quad \forall \mu \in \Gamma .
$$

Proof. 1) $0 \notin \Gamma$ (Proposition 6.7).

2) Let $\alpha, \beta \in \Gamma, \alpha+\beta \neq 0$. In order to prove that $\alpha+\beta \in \Gamma$ we make the following introductory remark. If $L(\kappa, \lambda)$ is solvable then it is abelian (Corollary 6.8). Hence we have $\kappa\left(L_{\lambda}\right)=0$. Otherwise the remark following Lemma 6.4 shows $\sigma(L(\kappa, \lambda))=$ $H(2 ; \underline{1} ; \Phi(\tau))^{(1)}$. Then all $i \kappa+j \lambda \neq 0$ are roots and $\mu\left(L_{\lambda}\right) \neq 0$ for all $\mu \in\langle\kappa, \lambda\rangle \backslash(0)$. Thus the following implications are true:

$$
\kappa\left(L_{\lambda}\right) \neq 0 \Longleftrightarrow \lambda\left(L_{\kappa}\right) \neq 0 \Longrightarrow\langle\kappa, \lambda\rangle \subset \Gamma \cup\{0\} .
$$

If $\beta\left(L_{\alpha}\right) \neq 0$ the preceding remark shows that $\alpha+\beta \in \Gamma$. Thus suppose $\beta\left(L_{\alpha}\right)=$ $0, \alpha\left(L_{\beta}\right)=0$. This implies that $L(\alpha, \beta)$ is abelian.

Suppose $\alpha, \beta$ are dependent. Since $L_{\alpha}$ does not annihilate $L$, there is a nonabelian 2 -section $L(\alpha, \gamma)$. The introductory remark yields that $G F(p)^{*} \alpha \subset \Gamma$. In particular, $\alpha+\beta \in \Gamma$.

Suppose that $\alpha, \beta$ are independent. The subalgebra $\sum_{\mu \in \Gamma} \sum_{i \geq 0} L_{\mu}{ }^{[p]^{i}}$ of the $p$-envelope $L_{p}$ of $L$ is closed under [p]-th powers and hence coincides with $L_{p}$. In particular, this subalgebra contains the torus $T$ of maximal toral rank. Since $\alpha+\beta$ is a nonzero linear form on $T$, there is $\gamma \in \Gamma$ such that $(\alpha+\beta)\left(L_{\gamma}^{[p]^{i}}\right) \neq 0$ for some $i \geq 0$. By symmetry we may assume $\alpha\left(L_{\gamma}[p]^{i}\right) \neq 0$, which by definition means $\alpha\left(L_{\gamma}\right) \neq 0$. Consider the section $L(\alpha, \beta, \gamma)$. $\gamma$ is independent of $\alpha, \beta$, since otherwise $L(\alpha, \beta, \gamma)=L(\alpha, \beta)$ would be abelian, contrary to the choice of $\gamma$. Hence we have the following setting:

$$
\alpha, \beta, \gamma \text { are independent, }
$$




$$
\begin{aligned}
& L(\alpha, \beta) \text { is abelian, } \\
& L(\alpha, \beta, \gamma) \text { is nonabelian and hence nonsolvable, } \\
& \alpha\left(L_{\gamma}\right) \neq 0, \quad \gamma\left(L_{\alpha}\right) \neq 0, \quad(\alpha+\beta)\left(L_{\gamma}\right) \neq 0 .
\end{aligned}
$$

a) Suppose $L(\alpha, \beta, \gamma)$ satisfies case 2$)$ of Theorem 6.1. Since $L(\alpha, \gamma)$ is nonabelian it is nonsolvable, and hence

$$
\sigma(L(\alpha, \beta, \gamma))=\sigma(L(\alpha, \gamma)) .
$$

Assume that for some $j \neq 0$ and some $i$ the linear combination $\mu:=i \alpha+j \beta$ is a root. In the present case $\sigma\left(L_{\mu}\right)=0$ holds, and hence $\sigma(L(\mu, \gamma))$ cannot be of Block type. Thus $L(\mu, \gamma)$ is solvable, whence it is abelian.

In particular, setting $j=1, i=0$, we observe that $\beta\left(L_{\gamma}\right)=0$.

Next suppose that some $\mu=i \alpha+j \beta(i, j \neq 0)$ is a root. Then $(i \alpha+j \beta)\left(L_{\gamma}\right)=$ $i \alpha\left(L_{\gamma}\right) \neq 0$, whence $L(\mu, \gamma)$ is nonabelian. This contradicts the preceding remark and hence shows that no linear form $i \alpha+j \beta(i, j \neq 0)$ is a root.

As $L_{\alpha}$ does not centralize $L$, the set $\Omega:=\left\{\lambda \in \Gamma \mid \lambda\left(L_{\alpha}\right) \neq 0\right\}$ is nonvoid. The simplicity of $L$ ensures the existence of $\delta \in \Omega$ such that $\left[L_{\delta}, L_{\beta-\delta}\right] \neq(0)$. Then $L(\delta, \beta)$ is nonabelian, proving $\beta\left(L_{\delta}\right) \neq 0$. The introductory remark yields $\beta-$ $\delta, 2(\beta-\delta) \in \Gamma$.

Since $\beta\left(L_{\alpha}\right)=0$ we have $(\beta-\delta)\left(L_{\alpha}\right)=\delta\left(L_{\alpha}\right) \neq 0$, showing that $\alpha+(\beta-\delta), \alpha+$ $2(\beta-\delta) \in \Gamma$.

If $(\alpha+\beta-\delta)\left(L_{\delta}\right) \neq 0$, then we obtain $\alpha+\beta=(\alpha+\beta-\delta)+\delta \in \Gamma$, contrary to the above.

If $(\alpha+\beta-\delta)\left(L_{\delta}\right)=0$, then $(\beta-\delta)\left(L_{\delta}\right)=-\alpha\left(L_{\delta}\right) \neq 0$. As above, we obtain the contradiction $\alpha+2 \beta=(\alpha+2(\beta-\delta))+2 \delta \in \Gamma$. Thus $L(\alpha, \beta, \gamma)$ cannot satisfy case 2) of Theorem 6.1 .

b) Suppose $L(\alpha, \beta, \gamma)$ satisfies case 3$)$ of Theorem 6.1. With the notations of Lemma 6.3 let $\kappa \in\langle\alpha, \beta, \gamma\rangle$ denote the linear form which vanishes on $\bigcup_{\mu} K_{\mu}$.

First we consider the case that $\kappa$ is a root. By asssumption no 2-section $K(\kappa, \mu)$ is of Block type, since otherwise $\kappa\left(K_{\mu}\right) \neq 0$. Hence $K(\kappa, \mu)$ is solvable. Then $L(\kappa, \mu)$ is solvable as well. Thus it is abelian. We obtain that $K_{\kappa}$ centralizes $K$, contradicting the fact that $K+R$ is semisimple.

Thus $\kappa$ is not a root. Since there are 3 independent roots on $K$, some

$$
\mu=k \kappa+i \alpha+j \gamma, \quad k \neq 0, \quad(i, j) \neq(0,0)
$$

is a root. We observe that $\mu\left(L_{\gamma}\right)=i \alpha\left(L_{\gamma}\right), \mu\left(L_{\alpha}\right)=j \gamma\left(L_{\alpha}\right)$. As $\alpha\left(L_{\gamma}\right) \neq 0$ and $\gamma\left(L_{\alpha}\right) \neq 0$, the introductory remark (applied several times) shows that

$$
\{r \kappa+s \alpha+t \gamma \mid r, s, t \in G F(p),(s, t) \neq(0,0)\}
$$

is contained in $\Gamma$.

Since $\alpha+\beta$ does not vanish on $L_{\gamma}, \alpha+\beta$ is contained in this set. Thus $\alpha+\beta \in \Gamma$.

c) Suppose $L(\alpha, \beta, \gamma)$ satisfies case 4$)$ of Theorem 6.1. Then the socle $S$ of $\sigma(L(\alpha, \beta, \gamma))$ satisfies $(A ; 3)$. Now Theorem 5.6 shows that $\alpha+\beta$ is a root.

3) Put $\Omega:=\left\{\mu \in \Gamma \mid \operatorname{dim} L_{\mu}\right.$ is minimal $\}$, and $I:=\sum_{\mu \in \Omega} L_{\mu}$. We prove that $I$ is an ideal of $L$. Take any $\mu \in \Omega$ and $\alpha \in \Gamma$. If $\mu\left(L_{\alpha}\right) \neq 0$, then there is $x \in L_{\alpha}$ with $\mu(x) \neq 0$, and this element acts invertibly on $\sum_{i \in G F(p)} L_{\mu+i \alpha}$. Consequently, $\operatorname{dim} L_{\mu+\alpha}=\operatorname{dim} L_{\mu}$, and $\mu+\alpha \in \Omega$.

If $\mu\left(L_{\alpha}\right)=0$ then $L(\alpha, \mu)$ is abelian, whence $\left[L_{\mu}, L_{\alpha}\right]=(0)$. 


\section{The Classification}

As a result of the above deliberations we may embed the Lie algebras under consideration into more convenient algebras. To that end we consider a finite dimensional vector space $M$ and a finite subgroup $G$ in $M^{*}$, the dual space of $M$. Put $M_{\alpha}:=\operatorname{ker} \alpha=\{m \in M \mid \alpha(m)=0\}$ for all $\alpha \in G$ and let $V(M, G)$ be the graded space

$$
V(M, G):=\bigoplus_{\mu \in G} M_{\mu}
$$

For the sake of clarity we write the $\mu$-part of $V(M, G)$ as $\left\{(m, \mu) \mid m \in M_{\mu}\right\}=$ $\left(M_{\mu}, \mu\right)$. Together with the product

$$
\left[(m, \alpha),\left(m^{\prime}, \beta\right)\right]:=\left(\alpha\left(m^{\prime}\right) m-\beta(m) m^{\prime}, \alpha+\beta\right),
$$

$V(M, G)$ becomes a Lie algebra.

For any root vector $x \in L_{\mu}$ let $x_{s} \in T$ denote the semisimple part of $x$ in $L_{p}$.

Theorem 7.1. Let $L$ be a simple Lie algebra satisfying $(A ; T R(L))$ and let $L_{p}$ be a semisimple p-envelope of $L$. Let $T$ denote a torus of $L_{p}$ of dimension $T R(L), \Gamma$ the set of roots of $T$ on $L$ and $G=\Gamma \cup\{0\}$ the $G F(p)$-vector space spanned by $\Gamma$. The mapping

$$
\begin{aligned}
\Psi: L & \longrightarrow V(T, G), \\
x & \longmapsto\left(-x_{s}, \alpha\right) \quad \forall x \in L_{\alpha},
\end{aligned}
$$

constitutes an injective Lie algebra homomorphism.

Proof. 1) Every root space $L_{\mu}$ is abelian (Corollary 6.8). We therefore have for arbitrary $x, y \in L_{\mu}$,

$$
(x+y)_{s}=x_{s}+y_{s}
$$

$[21,(2.3)]$. Thus $\Psi$ is linear on every root space and hence extends to a linear mapping on $L$.

2) In order to prove that $\Psi$ is a Lie homomorphism we take $x \in L_{\alpha}, y \in L_{\beta}$ and show that

$$
\gamma([x, y])=-\alpha(y) \gamma(x)+\beta(x) \gamma(y)
$$

for all roots $\gamma$, which satisfy $\gamma(x) \neq 0$. Once (5) is established, the definition of an extended root (namely $\gamma(x)=\gamma\left(x_{s}\right)$ ) yields that the element

$$
z:=[x, y]_{s}+\alpha(y) x_{s}-\beta(x) y_{s} \in T
$$

is annihilated by all $\gamma \in \Gamma$ with $\gamma(x) \neq 0$. As $\Omega:=\{\mu \in \Gamma \mid \mu(x) \neq 0\}$ is nonvoid (Proposition 6.7), the simplicity of $L$ implies that all roots vanish on $z$. Consequently, $z$ centralizes $L$ and hence $L_{p}$. As $L_{p}$ was chosen semisimple, this gives the desired result.

We will now prove (5). Take $\gamma \in \Gamma, \gamma(x) \neq 0$. The condition on $\gamma$ means that $L(\alpha, \gamma)$ is nonsolvable (Corollary 6.8).

(a) If $L(\alpha, \beta)$ is solvable, then according to Corollary 6.8

$$
[x, y]=0, \quad \alpha(y)=0, \quad \beta(x)=0 .
$$

Thus (5) is trivially satisfied in this case.

(b) From now on we assume that $L(\alpha, \beta)$ is nonsolvable. Suppose that $L(\alpha, \beta, \gamma)$ satisfies case 2$)$ of Theorem 6.1. The 2 -sections $L(\alpha, \beta)$ and $L(\alpha, \gamma)$ are not solvable. In the present case there is only one nonsolvable 2 -section. Hence $\gamma$ is dependent 
on $\alpha, \beta$. As ker $\sigma$ acts nilpotently on $L(\alpha, \beta)$, it is sufficient to prove (5) in $K=$ $\sigma(L(\alpha, \beta)) \cong H(2 ; \underline{1}, \Phi(\tau))^{(1)}$. We have shown in [14, (VII.3)] that there is a basis of root vectors $u_{\mu} \in K_{\mu}$ and a biadditive skewsymmetric form $f$ such that

$$
\left[u_{\lambda}, u_{\mu}\right]=f(\lambda, \mu) u_{\lambda+\mu}, \quad \forall \lambda, \mu \in \Gamma .
$$

Consequently,

$$
\begin{aligned}
\left(\operatorname{ad}\left[u_{\alpha}, u_{\beta}\right]\right)^{p}\left(u_{\gamma}\right) & =\left(\operatorname{ad} f(\alpha, \beta) u_{\alpha+\beta}\right)^{p}\left(u_{\gamma}\right) \\
& =f(\alpha, \beta)^{p} f(\alpha+\beta, \gamma)^{p} u_{\gamma} \\
& =(f(\alpha, \beta) f(\alpha, \gamma)+f(\alpha, \beta) f(\beta, \gamma))^{p} u_{\gamma} \\
\left(\operatorname{ad} u_{\alpha}\right)^{p}\left(u_{\gamma}\right) & =f(\alpha, \gamma)^{p} u_{\gamma}, \\
\left(\operatorname{ad} u_{\beta}\right)^{p}\left(u_{\gamma}\right) & =f(\beta, \gamma)^{p} u_{\gamma}, \\
\left(\operatorname{ad} u_{\alpha}\right)^{p}\left(u_{\beta}\right) & =f(\alpha, \beta)^{p} u_{\beta}, \\
\left(\operatorname{ad} u_{\beta}\right)^{p}\left(u_{\alpha}\right) & =f(\beta, \alpha)^{p} u_{\alpha} .
\end{aligned}
$$

The result on extended roots (Proposition 1.1) shows that

$$
\begin{aligned}
\gamma\left(\left[u_{\alpha}, u_{\beta}\right]\right) & =f(\alpha, \beta) f(\alpha, \gamma)+f(\alpha, \beta) f(\beta, \gamma), \\
\gamma\left(u_{\alpha}\right) & =f(\alpha, \gamma), \\
\gamma\left(u_{\beta}\right) & =f(\beta, \gamma), \\
\beta\left(u_{\alpha}\right) & =f(\alpha, \beta)=-f(\beta, \alpha)=-\alpha\left(u_{\beta}\right) .
\end{aligned}
$$

This is the result.

(c) Suppose that $L(\alpha, \beta, \gamma)$ satisfies case 3) of Theorem 6.1. As ker $\sigma$ is nilpotent, it is sufficient to prove (5) in $K$. According to Lemma 6.3, there is $\kappa \in T^{*}$ which vanishes on $\bigcup_{\mu} K_{\mu}$. Each root of $K$ is contained in $\langle\alpha, \beta, \kappa\rangle$. Put $\gamma=i \alpha+j \beta+k \kappa$. As (5) is linear in $\gamma$ and $\kappa$ vanishes everywhere, it is enough to prove (5) for $i \alpha+j \beta$ instead of $\gamma$. This has been done in the former case.

(d) Suppose that $L(\alpha, \beta, \gamma)$ satisfies case 4$)$ of Theorem 6.1. As in the former case it is sufficient to check (5) in $K$ (which according to Lemma 6.4 and Proposition 6.7 coincides with $S$ ). Theorem 5.6 applies and yields the result.

3) To prove injectivity of $\Psi$, take $\sum_{\mu \in \Gamma} x_{\mu} \in \operatorname{ker} \Psi$. By definition of $\Psi$ we have $x_{\mu} \in \operatorname{ker} \Psi$ for all $\mu$. Therefore $x_{\mu}$ is [p]-nilpotent and consequently acts nilpotently on $L$. Proposition 6.7 yields $x_{\mu}=0$ for all $\mu$, and therefore $x=0$.

We now consider $L=\bigoplus_{\mu \in \Gamma}\left(M_{\mu}, \mu\right)$ as a homogeneous subalgebra of $V(T, G)$. Put

$$
M:=\sum M_{\mu}, m:=\operatorname{dim} M, \text { and } L_{0}:=\left\{\sum\left(u_{\mu}, \mu\right) \mid \sum u_{\mu}=0\right\} .
$$

Corollary 6.9 in combination with Theorem 7.1 yields that $\operatorname{dim} M_{\mu}=k$ is independent of $\mu$.

Lemma 7.2. 1) $L_{0}$ is a subalgebra of $L$.

2) $L / L_{0} \cong M$ via $\varphi\left(\sum\left(u_{\mu}, \mu\right)\right)=\sum u_{\mu}$.

3) $L_{0}$ acts on $M$ via

$$
\left(\sum_{\lambda}\left(u_{\lambda}, \lambda\right)\right) \cdot v:=\sum_{\lambda} \lambda(v) u_{\lambda}
$$


Proof. 1) Suppose $\sum u_{\mu}=\sum v_{\mu}=0$. Then

$$
\left[\sum_{\lambda}\left(u_{\lambda}, \lambda\right), \sum_{\mu}\left(v_{\mu}, \mu\right)\right]=\sum_{\lambda, \mu}\left(\lambda\left(v_{\mu}\right) u_{\lambda}-\mu\left(u_{\lambda}\right) v_{\mu}, \lambda+\mu\right) .
$$

The result follows from the observation that

$$
\sum_{\lambda, \mu}\left(\lambda\left(v_{\mu}\right) u_{\lambda}-\mu\left(u_{\lambda}\right) v_{\mu}\right)=\sum_{\lambda} \lambda\left(\sum_{\mu} v_{\mu}\right) u_{\lambda}-\sum_{\mu} \mu\left(\sum_{\lambda} u_{\lambda}\right) v_{\mu}=0 .
$$

2 ) is obvious.

3) For $\sum_{\lambda}\left(u_{\lambda}, \lambda\right) \in L_{0},\left(v_{\mu}, \mu\right) \in L$ the multiplication in $L$ yields

$$
\begin{aligned}
{\left[\sum_{\lambda}\left(u_{\lambda}, \lambda\right),\left(v_{\mu}, \mu\right)\right] } & =\sum_{\lambda}\left(\lambda\left(v_{\mu}\right) u_{\lambda}, \lambda+\mu\right)-\sum_{\lambda}\left(\mu\left(u_{\lambda}\right) v_{\mu}, \lambda+\mu\right) \\
& \equiv \sum_{\lambda}\left(\lambda\left(v_{\mu}\right) u_{\lambda}, \lambda+\mu\right) \quad\left(\bmod L_{0}\right) .
\end{aligned}
$$

The definition of $\varphi$ gives the result.

Lemma 7.3. For all $\kappa, \lambda, \mu \in \Gamma$ the following are true.

1) $\kappa\left(M_{\kappa}\right)=0$.

2) Suppose $\lambda\left(M_{\kappa}\right) \neq 0$. Then

a) $M_{\lambda} \neq M_{\kappa}$;

b) $\kappa\left(M_{\mu}\right) \neq 0 \forall \mu \in\langle\kappa, \lambda\rangle \backslash\langle\kappa\rangle$;

c) $M_{\kappa} \cap \operatorname{ker} \lambda=M_{\kappa} \cap M_{\lambda}=M_{\lambda} \cap \operatorname{ker} \kappa$;

d) $M_{\mu} \subset M_{\kappa}+M_{\lambda}$ for all $\mu \in\langle\kappa, \lambda\rangle \cap \Gamma$;

e) if $\kappa, \lambda, \mu$ are $F$-independent on $M_{\kappa}+M_{\lambda}$, then $M_{\mu} \subset M_{\kappa}+M_{\lambda}$.

Proof. 1) follows from the fact that $\kappa\left(L_{\kappa}\right)=0 \forall \kappa \in \Gamma$.

2) (a) follows as $\lambda\left(M_{\lambda}\right)=0, \lambda\left(M_{\kappa}\right) \neq 0$.

(b) There is $x \in L_{\kappa}$ satisfying $\lambda(x) \neq 0$. Then $L(\kappa, \lambda)$ is nonabelian. Hence there is $y \in L_{\mu}$ with $\kappa(y) \neq 0$. Thus $\kappa\left(M_{\mu}\right) \neq 0$.

(c) According to (b) we have $\kappa\left(M_{\lambda-\kappa}\right) \neq 0$. Choose $v \in M_{\lambda-\kappa}$ with $\kappa(v)=1$. Then, for every $w \in M_{\kappa} \cap$ ker $\lambda$ one has $($ as $(\lambda-\kappa)(w)=0)$

$$
(w, \lambda)=[(w, \kappa),(v, \lambda-\kappa)] \in\left(M_{\lambda}, \lambda\right) .
$$

Thus we have $M_{\kappa} \cap \operatorname{ker} \lambda \subset M_{\kappa} \cap M_{\lambda} \neq M_{\kappa}$. A dimension argument proves that $M_{\kappa} \cap \operatorname{ker} \lambda=M_{\kappa} \cap M_{\lambda}$. Note that according to (b) $\kappa\left(M_{\lambda}\right) \neq 0$. Interchanging $\kappa$ and $\lambda$, we obtain the remaining assertion.

(d) Since $L(\kappa, \lambda)$ is of Block type, it is generated as an algebra by $L_{\kappa}+L_{\lambda}$. The definition of the multiplication in $V(T, G)$ then means that $M_{\mu}$ is contained in $M_{\kappa}+M_{\lambda}$.

(e) Suppose $\mu\left(M_{\kappa} \cap M_{\lambda}\right)=0$. According to (a) and (c) we have

$$
\begin{array}{ll}
M_{\kappa}=\left(M_{\kappa} \cap M_{\lambda}\right) \oplus F e_{\kappa}, & \lambda\left(e_{\kappa}\right)=1, \\
M_{\lambda}=\left(M_{\kappa} \cap M_{\lambda}\right) \oplus F e_{\lambda}, & \kappa\left(e_{\lambda}\right)=1 .
\end{array}
$$

As $\kappa, \lambda, \mu$ vanish on $M_{\kappa} \cap M_{\lambda}$, these linear forms are $F$-dependent on $M_{\kappa}+M_{\lambda}$. This contradiction shows that $\mu\left(M_{\kappa} \cap M_{\lambda}\right) \neq 0$.

Since $\mu\left(M_{\kappa}\right) \neq 0$ and $\mu\left(M_{\lambda}\right) \neq 0$, we may apply (c) for various cases to obtain

$$
\begin{aligned}
& M_{\mu} \cap \operatorname{ker} \kappa=M_{\kappa} \cap M_{\mu}=M_{\kappa} \cap \operatorname{ker} \mu, \\
& M_{\mu} \cap \operatorname{ker} \lambda=M_{\lambda} \cap M_{\mu}=M_{\lambda} \cap \operatorname{ker} \mu .
\end{aligned}
$$


Next suppose that $M_{\mu} \cap \operatorname{ker} \kappa=M_{\mu} \cap \operatorname{ker} \lambda$. This assumption yields

$$
M_{\kappa} \cap M_{\mu}=M_{\lambda} \cap M_{\mu} \subset(\operatorname{ker} \kappa) \cap(\operatorname{ker} \lambda) \cap(\operatorname{ker} \mu) .
$$

As

$$
\begin{aligned}
\operatorname{dim} & \left(M_{\kappa}+M_{\lambda}\right) / M_{\kappa} \cap M_{\mu} \\
\quad & \leq \operatorname{dim}\left(M_{\kappa} / M_{\kappa} \cap M_{\mu}\right)+\operatorname{dim}\left(M_{\lambda} / M_{\lambda} \cap M_{\mu}\right) \leq 2,
\end{aligned}
$$

the linear forms $\kappa, \lambda, \mu$ are $F$-linearly dependent on $M_{\kappa}+M_{\lambda}$, a contradiction. Hence

$$
M_{\mu} \cap \operatorname{ker} \kappa \neq M_{\mu} \cap \operatorname{ker} \lambda,
$$

whence

$$
M_{\mu} \subset M_{\mu} \cap \operatorname{ker} \kappa+M_{\mu} \cap \operatorname{ker} \lambda=M_{\kappa} \cap \operatorname{ker} \mu+M_{\lambda} \cap \operatorname{ker} \mu \subset M_{\kappa}+M_{\lambda} .
$$

Proposition 7.4. Assume $\operatorname{dim} M_{\mu}>1$ for all $\mu \in \Gamma$. Then

$$
M_{\mu}=M \cap \operatorname{ker} \mu \quad \forall \mu \in \Gamma .
$$

Proof. Let $\alpha \in \Gamma$ be arbitrary. Then $\alpha \neq 0$ (Corollary 6.9) and there is $\beta \in \Gamma$ such that $L(\alpha, \beta)$ has core $H(2 ; \underline{1} ; \Phi(\tau))^{(1)}$ (Theorem 1.5). In this case

$$
\alpha\left(M_{\beta}\right) \neq 0, \quad \beta\left(M_{\alpha}\right) \neq 0 .
$$

Set

$$
\Omega:=\left\{\mu \in \Gamma \mid \alpha, \beta, \mu \text { are } F-\text { independent on } M_{\alpha}+M_{\beta}\right\} .
$$

(a) Suppose $\Omega=\emptyset$. Then

$$
\left(M_{\alpha}+M_{\beta}\right) \cap(\operatorname{ker} \alpha) \cap(\operatorname{ker} \beta)=\left(M_{\alpha}+M_{\beta}\right) \cap(\operatorname{ker} \alpha) \cap(\operatorname{ker} \beta) \cap(\operatorname{ker} \mu)
$$

for all $\mu \in \Gamma$. Consequently,

$$
\begin{aligned}
M_{\alpha} \cap(\operatorname{ker} \beta) & \subset\left(M_{\alpha}+M_{\beta}\right) \cap(\operatorname{ker} \alpha) \cap(\operatorname{ker} \beta) \\
& =\left(M_{\alpha}+M_{\beta}\right) \cap \bigcap_{\mu \in \Gamma}(\operatorname{ker} \mu) \subset T \cap \bigcap_{\mu \in \Gamma}(\operatorname{ker} \mu)=(0) .
\end{aligned}
$$

This implies $\operatorname{dim} M_{\alpha}=1$, contradicting our assumption.

(b) Suppose $\Omega \neq \emptyset$. If $\gamma \in \Omega$ then Lemma 7.3(e) gives $M_{\gamma} \subset M_{\alpha}+M_{\beta}$. Moreover, the simplicity of $L$ implies

$$
L=\sum_{\mu \in \Omega} L_{\mu}+\sum_{\lambda, \mu \in \Omega}\left[L_{\lambda}, L_{\mu}\right]
$$

Thus for $\gamma \notin \Omega$ we have $L_{\gamma}=\sum_{\lambda, \gamma-\lambda \in \Omega}\left[L_{\lambda}, L_{\gamma-\lambda}\right]$. Then

$$
M_{\gamma} \subset \sum_{\lambda, \gamma-\lambda \in \Omega}\left(M_{\lambda}+M_{\gamma-\lambda}\right) \subset M_{\alpha}+M_{\beta}
$$

by Lemma $7.3(2 . \mathrm{d}, \mathrm{e})$.

(c) As a consequence of the above,

$$
M=\sum_{\gamma \in \Gamma} M_{\gamma}=M_{\alpha}+M_{\beta} .
$$

Thus Lemma 7.3(2.c) yields

$$
M \cap \operatorname{ker} \alpha=\left(M_{\alpha}+M_{\beta}\right) \cap \operatorname{ker} \alpha=M_{\alpha}+M_{\beta} \cap \operatorname{ker} \alpha=M_{\alpha} .
$$


We are now able to prove the classification results we are striving for. Let $L$ be a simple Lie algebra satisfying $(A ; k)$. The assumption $k=1$ implies that $L$ as a 1 -section is solvable, a contradiction. If $k=2$ then $L \cong H(2 ; \underline{1} ; \Phi(\tau))^{(1)}$ is of Hamiltonian type (Theorem 1.5(3)). We are now mainly interested in the case $k \geq 3$.

Theorem 7.5. Let $L$ be a simple Lie algebra satisfying $(A ; k)$. Suppose that $\operatorname{dim} L_{\mu}$ $\geq 2$ for all $\mu \in \Gamma$. Then $L$ is of Cartan type. More precisely,

$$
L \cong S(m ; \underline{n} ; \Phi(\tau))^{(1)}, \quad 3 \leq m \leq k=\sum_{i=1}^{m} n_{i} .
$$

Proof. Let $L_{p}$ denote a semisimple $p$-envelope of $L$ and $T \subset L_{p}$ a torus of maximal dimension $(=k)$. Note that $\operatorname{dim} L_{\mu}=\operatorname{dim} M_{\mu}=(\operatorname{dim} M)-1$ (by Proposition 7.4). Set $m:=\operatorname{dim} M \geq 3$. By definition, $M \subset T$ and hence $m \leq k$. The semisimplicity of $L_{p}$ implies that there are roots $\alpha_{1}, \ldots, \alpha_{m}$ such that

$$
M \cap \bigcap_{i=1}^{m} \operatorname{ker} \alpha_{i}=(0) .
$$

Choose a basis $q_{1}, \ldots, q_{m} \in M$ dual to $\alpha_{1}, \ldots, \alpha_{m}$. The action of $L_{0}$ on $M$ has been described in Lemma 7.2. With respect to the basis $\left(q_{1}, \ldots, q_{m}\right)$ the following elements of $L_{0}$ represent the respective matrices (for $i \neq j$ ):

$$
\begin{aligned}
\left(q_{i}, 2 \alpha_{j}\right)-\left(q_{i}, \alpha_{j}\right) & : E_{i j}, \\
\left(q_{i}-q_{j}, \alpha_{i}+\alpha_{j}\right)-\left(q_{i}, \alpha_{j}\right)+\left(q_{i}, \alpha_{i}\right) & : E_{i i}-E_{j j} .
\end{aligned}
$$

Next we compute traces. For $x=\sum_{\mu}\left(u_{\mu}, \mu\right) \in L_{0}$ we have $\sum_{\mu} u_{\mu}=0$. Express $u_{\mu}$ in terms of the $\alpha_{j}, q_{j}$ as

$$
u_{\mu}=\sum_{j=1}^{m} \alpha_{j}\left(u_{\mu}\right) q_{j}
$$

Then

$$
0=\mu\left(u_{\mu}\right)=\sum_{j=1}^{m} \alpha_{j}\left(u_{\mu}\right) \mu\left(q_{j}\right) .
$$

On the other hand,

$$
x . q_{i}=\sum_{\mu} \mu\left(q_{i}\right) u_{\mu}=\sum_{\mu, j} \mu\left(q_{i}\right) \alpha_{j}\left(u_{\mu}\right) q_{j},
$$

and hence (6) yields

$$
\text { trace } \operatorname{ad}_{L / L_{0}} x=\sum_{i=1}^{m} \sum_{\mu \in \Gamma} \mu\left(q_{i}\right) \alpha_{i}\left(u_{\mu}\right)=0 .
$$

Let $\left(L_{i}\right)_{i \geq-1}$ denote the standard filtration defined by $L_{0}$ :

$$
L_{-1}:=L, \quad L_{i+1}:=\left\{x \in L_{i} \mid[L, x] \subset L_{i}\right\} \quad(i \geq 0) .
$$

Thus it is proved that $L_{0} / L_{1} \cong \operatorname{ad}_{L / L_{0}} L_{0} \cong \mathfrak{s l}(m)$. We state as a consequence that $L / L_{0}$ is an irreducible $L_{0}$-module.

Note that there are exactly $p^{k}-1$ roots each of dimension $m-1$ (Corollary 6.9 and Proposition 7.4). Thus

$$
\operatorname{dim} L=(m-1)\left(p^{k}-1\right) .
$$


By definition $L_{1} / L_{2}$ embeds into $\operatorname{Hom}\left(L / L_{0}, L_{0} / L_{1}\right)$, and hence

$$
\begin{aligned}
\operatorname{dim} L_{2} & =\operatorname{dim} L-\left(\operatorname{dim} L / L_{0}+\operatorname{dim} L_{0} / L_{1}+\operatorname{dim} L_{1} / L_{2}\right) \\
& \geq(m-1)\left(p^{k}-1\right)-\left(m+\left(m^{2}-1\right)+m\left(m^{2}-1\right)\right)>0 .
\end{aligned}
$$

Therefore the recognition theorem applies and shows that $L \cong S(m ; \underline{n} ; \Psi)^{(1)}$ is of Cartan type.

The associated graded algebra satisfies the compatibility condition

$$
S(m ; \underline{n})^{(1)} \subset \operatorname{gr} L \subset S(m ; \underline{n}) .
$$

Then (cf. [21, (4.3.7)])

$$
(m-1)\left(p^{\sum n_{i}}-1\right) \leq(m-1)\left(p^{k}-1\right)
$$

whence $\sum n_{i} \leq k$. On the other hand, $S(m ; \underline{n} ; \Psi)^{(1)}$ is a subalgebra of $W(m ; \underline{n})$, and hence

$$
k=T R(L) \leq T R(W(m ; \underline{n}))=\sum n_{i} .
$$

According to [26] there are 3 isomorphism types of special algebras (see also $\S 5$ ).

(a) $S(m ; \underline{n})^{(1)}$ : The $p$-envelope is given by

$$
S(m ; \underline{n})^{(1)}+\sum_{i=1}^{m} \sum_{j_{i}=1}^{n_{i}-1} F D_{i}^{p^{j_{i}}}
$$

(cf. [6]). If $L \cong S(m ; \underline{n})^{(1)}$ then

$$
\operatorname{dim} L_{p} / L=\sum_{i=1}^{m}\left(n_{i}-1\right)<k
$$

and hence $T \cap L \neq(0)$. This contradicts Proposition 6.7.

(b) $S(m ; \underline{n} ; \Phi(\ell))^{(1)}$. Following the computations in [26] one obtains that this algebra is given by

$$
S(m ; \underline{n} ; \Phi(\ell))=\left\{E \in W(m ; \underline{n}) \mid E\left(\sum_{r=0}^{\infty}\left(x_{\ell}^{\left(p^{\left.n_{\ell}\right)}\right)}\right)^{(r)} \bigwedge_{j=1}^{m} d x_{j}\right)=0\right\} .
$$

Set $E_{t}:=D_{\ell}-x_{\ell}^{\left(p^{n_{\ell}}-1\right)} x_{t} D_{t}$ for $t \neq \ell$. It is easy to check that

$$
E_{t} \in S(m ; \underline{n} ; \Phi(\ell))^{(1)} \text { and } E_{t}^{p^{n_{\ell}}}=D_{\ell}^{p^{n_{\ell}}}-x_{t} D_{t} .
$$

Also

$$
\prod_{i \neq t} x_{i}^{\left(p^{n_{i}}-1\right)} D_{t}, x_{t} D_{t}-x_{\ell} D_{\ell} \in S(m ; \underline{n} ; \Phi(\ell)) \quad \forall t \neq \ell .
$$

Note that $D_{\ell}^{p^{n} \ell}=0$ when considered as a derivation on $A(m ; \underline{n})$. We now consider the $p$-envelope $L_{p}$ of $L=S(m ; \underline{n} ; \Phi(\ell))^{(1)}$ in Der $A(m ; \underline{n})$, and conclude from the above observation that $\sum_{i=1}^{m} F x_{i} D_{i} \subset L_{p}$. Then also

$$
\sum_{j \neq \ell} F \prod_{i \neq j} x_{i}^{\left(p^{n_{i}}-1\right)} D_{j} \subset S(m ; \underline{n} ; \Phi(\ell))^{(1)} .
$$

Consider the associated graded algebra gr $L$. The compatibility condition

$$
S(m ; \underline{n})^{(1)} \subset \text { gr } L \subset S(m ; \underline{n})
$$


gives

$$
\operatorname{gr} L \supset S(m ; \underline{n})^{(1)}+\sum_{j \neq \ell} F \prod_{i \neq j} x_{i}^{\left(p^{n_{i}}-1\right)} D_{j} .
$$

From $([21,(4.3 .7)])$ we conclude that

$$
\operatorname{dim} L \geq \operatorname{dim} S(m ; \underline{n})^{(1)}+(m-1)=(m-1) p^{\sum n_{i}} .
$$

As $\operatorname{dim} L=(m-1)\left(p^{\sum n_{i}}-1\right)$ we end up with a contradiction.

Thus the only possible case is the one claimed.

We now turn to the case that $\operatorname{dim} L_{\mu}=1$ for all $\mu \in \Gamma$.

Lemma 7.6. Let $L(\alpha, \beta, \gamma)$ be a 3-section as in case 3) or 4) of Theorem 6.1. Then there is a basis $\left(u_{\mu}\right)_{\mu \in \Gamma}$ of root vectors and a skewsymmetric biadditive form $f:(\Gamma \cup\{0\}) \times(\Gamma \cup\{0\}) \rightarrow F$ such that

$$
\left[u_{\lambda}, u_{\mu}\right]=f(\lambda, \mu) u_{\lambda+\mu} \quad \forall \alpha, \mu \in \Gamma .
$$

Proof. (a) Let $L(\alpha, \beta, \gamma)$ be as in case 3$)$ of Theorem 6.1 , and let $S=H(2 ; \underline{1} ; \Phi(\tau))^{(1)}$ $\otimes A(1 ; \underline{1})$ be the socle of the $T$-semisimple quotient $K$. Lemma 6.3 states that there is $\kappa$ such that $\kappa\left(K_{\mu}\right)=0$ for all $\mu \in \Gamma$. Then $L(\kappa, \mu)$ is not Hamiltonian and hence abelian (Corollary 6.8). Thus

$$
\left[L_{\lambda}, L_{\mu}\right]=(0) \text { if } \kappa, \lambda, \mu \text { are } G F(p) \text {-dependent. }
$$

Suppose that $\kappa, \lambda, \mu$ are not $G F(p)$-dependent. The image $R$ of $T$ intersects the $p$-envelope $S_{p}$ in a 2-dimensional torus $R_{0}$, and $C_{K}\left(R_{0}\right)=K(\kappa)$. Then $C_{K}\left(R_{0}\right) \subset$ $C(K)=(0)$. Hence $S$, being a minimal ideal of $K+R$, is $R$-simple. Therefore ([14, (IV.3)]) shows that $R$ is conjugate to $R_{0} \otimes F \oplus F \operatorname{Id} \otimes(1+x) \partial$, where $R_{0}$ is a maximal 2-dimensional torus in the $p$-envelope of $H(2 ; 1 ; \Phi(\tau))^{(1)}$. According to [14, (VII.3)] $S$ has an eigenvector basis $\left(e_{\lambda} \otimes(1+x)^{i}\right)$, where $\left(e_{\lambda}\right)$ is an eigenvector basis of $H(2 ; 1 ; \Phi(\tau))^{(1)}$ and the multiplication is given by a biadditive form $g$ :

$$
\left[e_{\lambda}, e_{\mu}\right]=g(\lambda, \mu) e_{\lambda+\mu} .
$$

Now all root spaces are 1-dimensional. Therefore

$$
\operatorname{rad}(L(\alpha, \beta, \gamma)+T) \subset \sum_{i \in G F(p)} L_{i \kappa}+T,
$$

and $L(\lambda, \mu)$ is mapped isomorphically onto $S(\lambda, \mu)$. We may now assume that

$$
L_{i \lambda+j \mu+k \kappa}=F e_{i \lambda+j \mu} \otimes(1+x)^{k}
$$

and multiplication is given by

$$
\begin{aligned}
{\left[e_{i \lambda+j \mu} \otimes\right.} & \left.(1+x)^{k}, e_{i^{\prime} \lambda+j^{\prime} \mu} \otimes(1+x)^{k^{\prime}}\right] \\
& =\left(i j^{\prime}-i^{\prime} j\right) g(\lambda, \mu) e_{\left(i+i^{\prime}\right) \lambda+\left(j+j^{\prime}\right) \mu} \otimes(1+x)^{k+k^{\prime}} .
\end{aligned}
$$

Set

$$
f(\kappa, \lambda):=f(\kappa, \mu):=0, \quad f(\lambda, \mu):=g(\lambda, \mu) .
$$

(b) Let $L(\alpha, \beta, \gamma)$ be as in case 4$)$ of Theorem 6.1. Since $C_{L}(T)=(0)$ and $\operatorname{dim} L_{\mu}=1 \forall \mu$, we have $L(\alpha, \beta, \gamma)=S \cong H(2 ;(2,1) ; \Phi(\tau))^{(1)}$. Proposition 5.4 yields the result. 
Lemma 7.7. Let $\alpha, \beta, \gamma \in \Gamma$, and let $v_{\mu} \in L_{\mu}$ (for $\mu=\alpha, \beta, \gamma, \beta+\gamma$ ) be root vectors satisfying

$$
\begin{aligned}
& \alpha\left(v_{\beta}\right)=-\beta\left(v_{\alpha}\right) \neq 0 \\
& \alpha\left(v_{\gamma}\right)=-\gamma\left(v_{\alpha}\right) \neq 0 .
\end{aligned}
$$

Then

$$
\gamma\left(v_{\beta}\right)=-\beta\left(v_{\gamma}\right)
$$

If in addition $\alpha\left(v_{\beta+\gamma}\right)=-(\beta+\gamma) v_{\alpha} \neq 0$, then

$$
\left[v_{\beta}, v_{\gamma}\right]=\gamma\left(v_{\beta}\right) v_{\beta+\gamma}
$$

Proof. Suppose that $\alpha, \beta, \gamma$ are $G F(p)$-independent.

1) The 3 -section $L(\alpha, \beta, \gamma)$ contains two different nonabelian 2-sections $L(\alpha, \beta)$, $L(\alpha, \gamma)$. Then these 2 -sections are nonsolvable. Hence the $T$-semisimple quotient of $L(\alpha, \beta, \gamma)$ is as in cases 3$)$ or 4$)$ of Theorem 6.1. The preceding lemma shows that there is a basis $\left(u_{\mu}\right)$ of $L(\alpha, \beta, \gamma)$ and a form $f$ such that

$$
\left[u_{\mu}, u_{\kappa}\right]=f(\mu, \kappa) u_{\mu+\kappa} \quad \forall \mu, \kappa \in\langle\alpha, \beta, \gamma\rangle .
$$

Find $s_{\mu} \in F \backslash\{0\}$ with $v_{\mu}=s_{\mu} u_{\mu}(\mu=\alpha, \beta, \gamma, \beta+\gamma)$. Then

$$
\left[v_{\mu}, u_{\kappa}\right]=s_{\mu} f(\mu, \kappa) u_{\kappa+\mu} \text { for } \kappa \in\langle\alpha, \beta, \gamma\rangle,
$$

and the eigenvalue of $\left(\operatorname{ad} v_{\mu}\right)^{p}$ occurring here is

$$
\kappa\left(v_{\mu}\right)^{p}=\left(s_{\mu} f(\mu, \kappa)\right)^{p} .
$$

Due to our assumption, for $\mu=\beta, \gamma$ we obtain

$$
s_{\mu} f(\mu, \alpha)=\alpha\left(v_{\mu}\right)=-\mu\left(v_{\alpha}\right)=-s_{\alpha} f(\alpha, \mu)=s_{\alpha} f(\mu, \alpha) \neq 0 .
$$

Consequently $s_{\alpha}=s_{\beta}=s_{\gamma}=: s$, and hence

$$
\gamma\left(v_{\beta}\right)=s f(\beta, \gamma)=-s f(\gamma, \beta)=-\beta\left(v_{\gamma}\right) .
$$

2) If $\alpha\left(v_{\beta+\gamma}\right)=-(\beta+\gamma)\left(v_{\alpha}\right) \neq 0$, then the above computations show that $s_{\beta+\gamma}=s_{\alpha}=s$ and

$$
\left[v_{\beta}, v_{\gamma}\right]=s^{2}\left[u_{\beta}, u_{\gamma}\right]=s^{2} f(\beta, \gamma) u_{\beta+\gamma}=\gamma\left(v_{\beta}\right) v_{\beta+\gamma} .
$$

If $\alpha, \beta, \gamma$ are $G F(p)$-dependent, then the 2 -section $L(\alpha, \beta, \gamma)$ is nonabelian. Then $L(\alpha, \beta, \gamma) \cong H(2 ; \underline{1} ; \Phi(\tau))^{(1)}$. Now proceed as in the former case.

We are now ready to prove the second (and final) classification theorem.

Theorem 7.8. Let $L$ be a simple Lie algebra satisfying $(A ; k)$ for some $k \geq 2$. Suppose that $\operatorname{dim} L_{\mu}=1$ for all $\mu \in \Gamma$. Then there is a skewsymmetric biadditive form $f:(\Gamma \cup\{0\}) \times(\Gamma \cup\{0\}) \rightarrow F$ and an eigenvector basis $\left(u_{\mu}\right)_{\mu \in \Gamma}$ satisfying $L_{\mu}=F u_{\mu}$ and

$$
\left[u_{\mu}, u_{\lambda}\right]=f(\mu, \lambda) u_{\mu+\lambda} \text { for all } \lambda, \mu \in \Gamma .
$$

Thus $L \cong L(\Gamma \cup\{0\}, 0, f)^{(1)}$ is a BLOCK algebra.

Proof. 1) Take $\alpha \in \Gamma$ arbitrary and set

$$
\Omega:=\left\{\mu \in \Gamma \mid \mu\left(L_{\alpha}\right) \neq 0\right\} \neq \emptyset .
$$

Fix $u_{\alpha} \in L_{\alpha} \backslash\{0\}$. For $\mu \in \Omega$ we choose $u_{\mu} \in L_{\mu}$ with

$$
\mu\left(u_{\alpha}\right)=-\alpha\left(u_{\mu}\right) .
$$

Note that $\mu\left(u_{\alpha}\right) \neq 0$. 
2) If $\kappa \in \Omega$, then Lemma 7.7 yields

$$
\mu\left(u_{\kappa}\right)=-\kappa\left(u_{\mu}\right) .
$$

Consider any $\kappa \notin \Omega$. As $L$ is simple and $\Omega \neq \emptyset$, we have

$$
L_{\kappa}=\sum_{\mu, \kappa-\mu \in \Omega}\left[L_{\mu}, L_{\kappa-\mu}\right] .
$$

Let $\mu, \lambda$ be roots such that $\mu, \lambda, \kappa-\mu, \kappa-\lambda \in \Omega$ and

$$
\left[L_{\mu}, L_{\kappa-\mu}\right]=L_{\kappa}, \quad\left[L_{\lambda}, L_{\kappa-\lambda}\right]=L_{\kappa} .
$$

(Recall that since $\operatorname{dim} L_{\kappa}=1$ this just means $\left[L_{\mu}, L_{\kappa-\mu}\right] \neq(0),\left[L_{\lambda}, L_{\kappa-\lambda}\right] \neq(0)$.) Lemma 7.7 with $\beta=\mu, \gamma=\lambda$ yields

$$
\mu\left(u_{\lambda}\right)=-\lambda\left(u_{\mu}\right) .
$$

Similarly, we obtain

$$
\begin{aligned}
& \lambda\left(u_{\kappa-\lambda}\right)=-(\kappa-\lambda)\left(u_{\lambda}\right)=-\kappa\left(u_{\lambda}\right), \\
& \mu\left(u_{\kappa-\lambda}\right)=-(\kappa-\lambda)\left(u_{\mu}\right) .
\end{aligned}
$$

Next consider the 3 -section $L(\kappa, \lambda, \mu)$. As $L(\kappa, \lambda), L(\kappa, \mu)$ are nonabelian, they are of Hamiltonian type. Then there is $u_{\kappa} \in L_{\kappa}$ satisfying $\mu\left(u_{\kappa}\right)=-\kappa\left(u_{\mu}\right) \neq 0$. We also obtain $\kappa\left(u_{\lambda}\right) \neq 0, \lambda\left(u_{\kappa}\right) \neq 0$. If $\mu\left(u_{\lambda}\right) \neq 0$, we obtain from Lemma 7.7 with $\alpha=\mu$ that

$$
\lambda\left(u_{\kappa}\right)=-\kappa\left(u_{\lambda}\right) .
$$

If $\mu\left(u_{\lambda}\right)=0$, then $\lambda\left(u_{\mu}\right)=0$ and

$$
\kappa\left(u_{\mu}\right)=(\kappa-\lambda)\left(u_{\mu}\right)=-\mu\left(u_{\kappa-\lambda}\right) \neq 0 .
$$

Again applying Lemma 7.7 (with $\alpha=\mu, \beta=\kappa, \gamma=\kappa-\lambda$ ) we get $0 \neq(\kappa-\lambda)\left(u_{\kappa}\right)=$ $-\kappa\left(u_{\kappa-\lambda}\right)$, and (with $\alpha=\kappa-\lambda, \beta=\lambda, \gamma=\kappa$ )

$$
\lambda\left(u_{\kappa}\right)=-\kappa\left(u_{\lambda}\right) .
$$

Thus $u_{\kappa}$ is defined independently of the choice of $\mu \in \Omega$ by the condition

$$
\mu\left(u_{\kappa}\right)=-\kappa\left(u_{\mu}\right)
$$

for any arbitrary $\mu \in \Omega, \mu\left(L_{\kappa}\right) \neq 0$. Thus we have defined nonzero eigenvectors $u_{\mu}$ for all $\mu \in \Gamma$.

3) For $\lambda, \mu \in \Gamma$ set

$$
f(\lambda, \mu):=\mu\left(u_{\lambda}\right), \quad f(0, \mu):=f(\lambda, 0):=0 .
$$

By definition $f$ is additive in the second argument. We have to prove that

$$
\left[u_{\lambda}, u_{\mu}\right]=f(\lambda, \mu) u_{\lambda+\mu} \quad \forall \lambda, \mu \in \Gamma .
$$

From this it will immediately follow that $f$ is skewsymmetric.

Take $\lambda, \mu \in \Gamma$ arbitrary. If $L(\lambda, \mu)$ is abelian, then we are done. Otherwise $\mu\left(u_{\lambda}\right) \neq 0, \mu\left(u_{\lambda+\mu}\right) \neq 0$. There is $\rho \in \Omega$ with

$$
\rho\left(u_{\mu}\right) \neq 0,
$$

since otherwise $\gamma\left(u_{\mu}\right)=0$ for all $\gamma \in \Gamma$, contradicting Proposition 6.7. As $p \geq 5$, the set $\rho+G F(p) \mu$ contains an element (which we again call $\rho$ ) satisfying

$$
\rho \in \Omega, \quad \rho\left(u_{\mu}\right) \neq 0, \quad \rho\left(u_{\lambda}\right) \neq 0, \quad \rho\left(u_{\lambda+\mu}\right) \neq 0 .
$$


Thus we have

$$
\rho\left(u_{\lambda}\right)=-\lambda\left(u_{\rho}\right) \neq 0, \rho\left(u_{\mu}\right)=-\mu\left(u_{\rho}\right) \neq 0, \rho\left(u_{\lambda+\mu}\right)=-(\lambda+\mu) u_{\rho} \neq 0 .
$$

The second part of Lemma 7.7 yields the desired result.

\section{REFERENCES}

[1] G.M. Benkart and T. Gregory, Graded Lie algebras with classical reductive null component, Math. Ann. 285 (1989), 85-98. MR 90j:17051

[2] R.E. Block and R.L. Wilson, The simple Lie-p-algebras of rank two, Ann. of Math. 115 (1982), 93-168. MR 83j: 17008

[3] R.E. Block and R.L. Wilson, Classification of the restricted simple Lie algebras, J. Algebra 114 (1988), 115-259. MR 89e: 17014

[4] G.M. Benkart et al., Isomorphism classes of Hamiltonian Lie algebras. In: G.M. Benkart and J.M. Osborn (eds.), Lie Algebras, Madison 1987, Lecture Notes in Math. 1373 (1989), Springer, Berlin-New York, 42 - 57. MR 91e:17014

[5] H.J. Chang, Über Wittsche Lie-Ringe, Abh. Math. Sem. Universität Hamburg 14 (1941), 151 - 184. MR 3:101a

[6] M.J. Celousov, Derivations of Lie algebras of Cartan type, Izv. Vysš. Učebn. Zaved. Matematika 98 (1970), 126 - 134. MR 43:6273

[7] V.G. Kac, Description of filtered Lie algebras with which graded Lie algebras of Cartan type are associated, Izv. Akad. Nauk SSSR Ser. Math. 38 (1974), 800 - 834; Errata, 40 (1976), 1415 [Russian]; Math. USSR-Izv. 8 (1974), 801 - 835; Errata, 10 (1976), 1339 [English transl.] MR 51:5685; MR 55:3008

[8] N.A. Koreshkov, On irreducible representations of Hamiltonian algebras of dimension $p^{2}-2$, Izvestija VUZ. Matematika 22 (1978), 37 - 46. [Russian]; Soviet Math (Iz. VUZ) 22 (1978), no. 10, 28-34 [English transl.] MR 81c:17020

[9] M.I. Kuznetsov, Simple modular Lie algebras with a solvable maximal subalgebra, Mat. Sb. 101 (1976), 77 - 86 [Russian]; Math. USSR-Sb. 30 (1976), 68 - 76 [English transl.] MR 54:1035b

[10] R.H. Oehmke, On a class of Lie algebras, Proc. Amer. Math. Soc. 16 (1965), 1107 - 1113 MR 33:157

[11] H. Strade, The Absolute Toral Rank of a Lie Algebra. In: G.M. Benkart and J.M. Osborn (eds.), Lie Algebras, Madison 1987, Lecture Notes in Math. 1373 (1989), Springer, BerlinNew York, 1-28. MR 90m:17029

[12] H. Strade, The Classification of the Simple Modular Lie Algebras: I. Determination of the two-sections, Ann. of Math. 130 (1989), 643-677. MR 91a:17023

[13] H. Strade, Lie Algebra Representations of Dimension $<p^{2}$, Trans. Amer. Math. Soc. 319 (1990), 689-709. MR 90j:17015

[14] H. Strade, The Classification of the Simple Modular Lie Algebras: II. The Toral Structure, J. Algebra 151 (1992), 425-475. MR 93j:17042

[15] H. Strade, New Methods for the Classification of Simple Modular Lie Algebras, Mat. Sbornik 181 (1990), 1391-1402 [Russian]; Math. USSR Sbornik 71 (1992), 235-245 [English transl.] MR 92d: 17020

[16] H. Strade, Representations of the $\left(p^{2}-1\right)$-dimensional Lie Algebras of R.E. Block, Can. J. Math. 43 (1991), 580-616. MR 92g:17025

[17] H. Strade, The Classification of the Simple Modular Lie Algebras: III. Solution of the Classical Case, Ann. of Math. 133 (1991), 577-604. MR 92g:17024

[18] H. Strade, The Classification of the Simple Modular Lie Algebras: IV. Determining the Associated Graded Algebra, Ann. of Math. 138 (1993), 1-59. MR 94k:17039

[19] H. Strade, The Classification of the Simple Modular Lie Algebras: V. Algebras with Hamiltonian Two-sections, Abh. Math. Sem. Univ. Hamburg 64 (1994), 167 - 202. MR 95h:17023

[20] H. Strade, Representations of Derivation Simple Algebras, AMS1P Studies in Advanced Mathematics 4 (1997), 127-142.

[21] H. Strade and R. Farnsteiner, Modular Lie Algebras and Their Representations, Marcel Dekker Textbooks and Monographs 116 (1988). MR 89h:17021

[22] H. Strade and R.L. Wilson, Classification of Simple Lie Algebras over Algebraically Closed Fields of Prime Characteristic, Bull. Amer. Math. Soc. 24 (1991), 357-362. MR 91i:17026 
[23] B.Yu. Weisfeiler, On the Structure of the Minimal Ideals of Some Graded Lie Algebras in Characteristic $p>0$, J. Algebra 53 (1978), 344-361. MR 80b:17011

[24] B.Yu. Weisfeiler, On subalgebras of simple Lie algebras of characteristic $p>0$, Trans. Amer. Math. Soc. 286 (1984), 471 - 503. MR 86h:17012

[25] R.L. Wilson, Simple Lie Algebras of Toral Rank One, Trans. Amer. Math. Soc. 236 (1978), 287-295. MR 57:3205

[26] R.L. Wilson, Simple Lie Algebras of Type S, J. Algebra 62 (1980), 292 - 298. MR 81c:17019

[27] D.J. Winter, On the toral structure of Lie $p$-algebras, Acta Math. 123 (1969), 69 -81. MR 40:4326

Mathematische Seminar Universität Hamburg, 20146 Hamburg, Germany

E-mail address: strade@math.uni-hamburg.de 